\title{
The experimental set-up of the RIB in-flight
}

\section{facility EXOTIC}

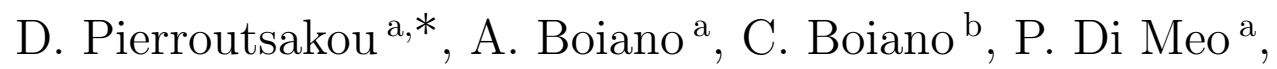
M. La Commara ${ }^{\mathrm{c}, \mathrm{a}}$, C. Manea ${ }^{\mathrm{d}}$, M. Mazzocco ${ }^{\mathrm{e}, \mathrm{d}}$,

M. Nicoletto ${ }^{\text {d }}$, C. Parascandolo ${ }^{a}$, C. Signorini ${ }^{\mathrm{e}, \mathrm{d}}$, F. Soramel $^{\mathrm{e}, \mathrm{d}}$,

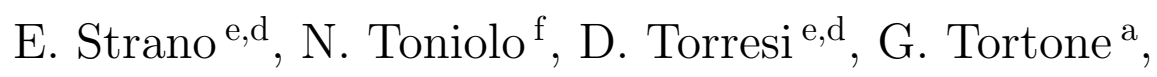

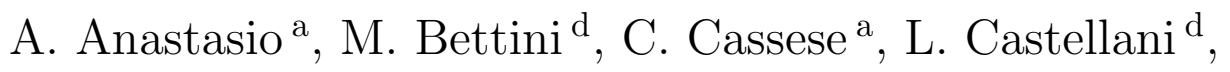

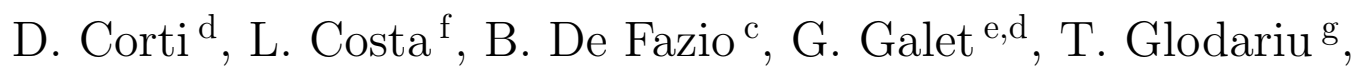
J. Grebosz $^{\text {h }}$, A. Guglielmetti ${ }^{\mathrm{i}, \mathrm{b}}$, P. Molini ${ }^{\mathrm{e}, \mathrm{d}}$, G. Pontoriere ${ }^{\mathrm{a}}$,

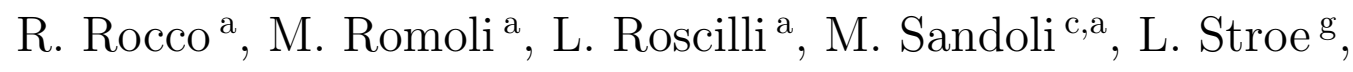
M. Tessaro ${ }^{\mathrm{d}}$, P.G. Zatti ${ }^{\mathrm{d}}$

${ }^{\mathrm{a}}$ INFN - Sezione di Napoli, Via Cintia, I-80126 Napoli, Italy ${ }^{\mathrm{b}}$ INFN - Sezione di Milano, Via Celoria 16, I-20133 Milano, Italy

${ }^{\mathrm{c}}$ Dipartimento di Fisica, Università di Napoli, Via Cintia, I-80126 Napoli, Italy ${ }^{\mathrm{d}}$ INFN - Sezione di Padova, Via Marzolo 8, I-35131 Padova, Italy

e Dipartimento di Fisica e Astronomia, Università di Padova, Via Marzolo 8, I-35131 Padova, Italy 
${ }^{\mathrm{f}} I N F N$ - LNL, Viale dell'Università 2, I-35020 Legnaro (PD), Italy

g NIPNE, Str. Reactorului no.30, P.O. BOX MG-6, Bucharest-Magurele,

\title{
Romania
}

h IFJ PAN, ul. Radzikowskiego 152, 31-342 Kraków, Poland

i Dipartimento di Fisica, Università di Milano, Via Celoria 16, I-20133, Milano,

$$
\text { Italy }
$$

\begin{abstract}
We describe the experimental set-up of the Radioactive Ion Beam (RIB) in-flight facility EXOTIC consisting of: a) two position-sensitive Parallel Plate Avalanche Counters (PPACs), dedicated to the event-by-event tracking of the produced RIBs and to time of flight measurements; b) the new high-granularity compact telescope array EXPADES (EXotic PArticle DEtection System), designed for nuclear physics and nuclear astrophysics experiments employing low-energy light RIBs. EXPADES consists of eight $\Delta E-E_{r e s}$ telescopes arranged in a cylindrical configuration around the target. Each telescope is made up of two Double Sided Silicon Strip Detectors (DSSSDs) with a thickness of $40 / 60 \mu \mathrm{m}$ and $300 \mu \mathrm{m}$ for the $\Delta E$ and $E_{\text {res }}$ layer, respectively. Additionally, eight ionization chambers were constructed to be used as an alternative $\Delta E$ stage or, in conjunction with the entire DSSSD array, to build up more complex triple telescopes. New low-noise multi-channel chargesensitive preamplifiers and spectroscopy amplifiers, associated with constant fraction discriminators, peak-and-hold and Time to Amplitude Converter circuits were developed for the electronic readout of the $\Delta E$ stage. Application Specific Inte-
\end{abstract}


grated Circuit-based electronics was employed for the treatment of the $E_{\text {res }}$ signals. An 8-channel, 12-bit multi-sampling $50 \mathrm{MHz}$ Analog to Digital Converter, a Trigger Supervisor Board for handling the trigger signals of the whole experimental set-up and an ad-hoc data acquisition system were also developed. The performance of the PPACs, EXPADES and of the associated electronics was obtained offline with standard $\alpha$ calibration sources and in-beam by measuring the scattering process for the systems ${ }^{17} \mathrm{O}+{ }^{58} \mathrm{Ni}$ and ${ }^{17} \mathrm{O}+{ }^{208} \mathrm{~Pb}$ at incident energies around their respective Coulomb barriers and, successively, during the first experimental runs with the RIBs of the EXOTIC facility.

Key words: Radioactive Ion Beams, Tracking and position-sensitive detectors, Charged-particle detection array, Low-noise electronics, ASIC electronics, Data conversion and acquisition

PACS: 25.60.Bx, 29.40.Gx, 29.40.Wk, 29.40.Cs, 07.50.-e, 0.7.05.Hd

\section{$1 \quad 1 \quad$ Introduction}

2 The growing availability of Radioactive Ion Beams (RIBs) worldwide has

3 opened up new scenarios and challenges in nuclear physics. Experiments with

4 radioactive (exotic) nuclei allow to explore the properties of isotopes that have

5 a proton-to-neutron ratio very different from the stable ones, measure cross

6 sections of important reactions for the stellar nucleosynthesis occurring in ex-

7 plosive astrophysical environments, constrain the isospin-dependent nucleon-

\footnotetext{
* pierroutsakou@na.infn.it
} 
8 nucleon interaction in neutron-rich nuclei and in neutron stars, synthesize

9 superheavy elements and test physics beyond the standard model. Moreover,

10 radioisotopes are being used for condensed matter atomic physics and life-

11 science studies.

12 While several large-scale RIB facilities are actually operating at RIKEN [1], 13 NSCL/MSU [2], GANIL (France) [3], GSI [4], CERN (ISOLDE) [5], TRI-

14 UMF (ISAC) [6] and small-scale facilities like Twinsol in Notre Dame Uni15 versity (USA) [7], RIBRAS (Brasil) [8], JYFL (Jyvaskyla, Finland) [9], CRIB 16 (Japan) $[10 ; 11 ; 12]$, EXOTIC (LNL-INFN, Italy) $[13 ; 14 ; 15 ; 16 ; 17]$, future in17 frastructures like SPES (LNL-INFN, Italy), SPIRAL2 (France), HIE-ISOLDE ${ }_{18}$ (CERN), FRIB (USA), FAIR (Germany), EURISOL (Europe) are aimed at 19 delivering RIBs with the highest intensity and purity and with good ion optical 20 quality for investigating unreachable parts of the nuclear chart.

${ }_{21}$ Along with the construction of new RIB infrastructures, a continuous devel22 opment of detection arrays is under way. Depending on the radioactive ion 23 incident energy and on the class of reactions to be studied, different experi24 mental set-ups were built for the detection of charged particles. To mention 25 some of these set-ups, MUST [18] and MUST2 [19], TIARA [20], LASSA [21], ${ }_{26}$ HIRA [22] are dedicated mainly to the study of nuclear reactions with light ${ }_{27}$ targets in inverse kinematics, LEDA [23] for nuclear physics and nuclear astrophysics experiments or the GLORIA array [24], for the study of reaction 29 mechanisms induced by light projectiles on heavy targets. 
In the present paper we describe the experimental set-up designed primarily to fully exploit the low-energy light RIBs delivered by the in-flight facility EXOTIC and consisting of: (a) the RIB tracking system and (b) EXPADES [25; 26], a new charged-particle telescope array. The envisioned experimental program employing the described set-up aims at:

(1) studying reaction mechanisms induced by light exotic nuclei impinging on medium- and heavy-mass targets at incident energies near the Coulomb barrier. In this energy range, the peculiar features of exotic nuclei, such as excess of neutrons or protons, low binding energy, halo structure, neutron or proton dominated surface, influence the elastic scattering and the fusion process giving a picture that is rather different from that of well bound species (for a review see for instance [27]). In the considered measurements the charged products emitted in direct nuclear reactions (elastic and inelastic scattering, nucleon transfer, breakup of the weakly bound projectile) and the light charged particles emitted in fusion-evaporation reactions should be charge and mass identified. A FWHM energy resolution of $\sim 250-400 \mathrm{keV}$ is needed in the most demanding cases for discriminating the elastic from the inelastic scattering of the projectile from the target, depending on the considered colliding nuclei: $\sim 250(400) \mathrm{keV}$ for a ${ }^{11} \mathrm{Be}\left({ }^{17} \mathrm{~F}\right)$ projectile impinging on a ${ }^{58} \mathrm{Ni}$ or ${ }^{208} \mathrm{~Pb}$ target. A large detection solid angle is requested to compensate the low RIB intensity, in the most favorable cases limited to a few orders of magnitude less than 
typical stable beams, and to allow detection of coincident breakup particles emitted at large relative angles while a high granularity would allow detection of coincident breakup particles emitted at small relative angles. A FWHM time resolution of $\sim 1-1.5 \mathrm{~ns}$ is sufficient for discriminating protons, $\alpha$ particles and heavy-ions for flight paths larger than $10 \mathrm{~cm}$ and for the event-by event rejection of contaminant beams. It has to be noticed here, that for nuclear reactions induced by in-flight produced RIBs, the overall experimental energy resolution is often limited by the energetic spread of the RIB and by the energy loss and energy straggling of the ions in the target that should be thick enough to compensate the low intensity of the beam;

(2) studying $\alpha$ clustering phenomena in light exotic nuclei [28], employing the Thick Target Inverse Kinematic (TTIK) scattering technique [29], with the RIB impinging on a ${ }^{4} \mathrm{He}$ gas target. The pressure of the gas is tuned such that the RIB completely stops in the gas while the energetic recoiling light target nuclei, due to their low-rate of energy loss, can traverse the gas and be recorded by the detectors. The TTIK method is particularly useful for measurements with low-intensity RIBs since it allows to measure the elastic scattering excitation function over a wide energy range by using a single beam energy. The experimental requirements for the detection array are: a good energy resolution, high granularity to reconstruct the interaction point and the beam energy at the interaction point and light particle identification. A FWHM time resolution of $\sim 1-1.5 \mathrm{~ns}$ is 
enough for separating elastic scattering from other processes in most of the cases. It is worthnoting that the TTIK method helps improving the overall experimental energy resolution because of the transformation from the laboratory to the center-of-mass reference frame [30];

(3) performing measurements of astrophysical interest with RIBs impinging on solid or gas light targets in inverse kinematics: among the different processes of stellar nucleosynthesis forming elements heavier than ${ }^{9} \mathrm{Be}$, the rapid proton-capture and $\alpha$ p processes, occurring in explosive astrophysical environments such as novae, x-ray bursters and type Ia supernovae, are those than can be investigated by using the EXOTIC RIBs. Moreover, experiments based on the Trojan Horse Method [31] are considered. In the latter measurements, two among the three charged reaction products in the final state need to be detected with a $\sim 2 \%$ FWHM energy resolution and a FWHM angular resolution better than $\sim 1^{\circ}[32]$.

To summarize, the design of a high-performance detection system suitable for the above mentioned experiments must meet several requirements:

(a) event-by-event beam tracking capabilities to account for the typical poor emittance of in-flight produced RIBs in conjuction with a good time resolution for TOF measurements and a fast signal for handling counting rates up to $10^{6} \mathrm{~Hz}$;

(b) charge and mass identification of the reaction products with the highest achievable energy resolution; 
(c) a solid angle coverage as large as possible;

(d) high segmentation to achieve good angular resolution and for reducing pile up events and low-energy events coming from the radioactive decay of the elastically scattered projectiles;

(e) flexibility in order to be suitable for different experimental needs.

Requisite (a) can be achieved by employing, at suitable positions along the beam-line, ad-hoc designed fast and high-transparency tracking detectors, providing the event-by-event reconstruction of the position hit on the reaction target, along with the reference time for TOF measurements. Requisite (b) can be fulfilled by using particle detector telescopes through the $\Delta E-E_{\text {res }}$ and/or the TOF technique. Requirements (c) and (d) can be matched by using large-area high-granularity Double Sided Silicon Strip Detectors (DSSSDs), associated with Ionization Chambers (ICs), in a closely-packed configuration around the reaction target position. Requirement (e) can be matched by having a modular and expandable array, the possibility to change the effective thickness of the $\Delta E$ detector, the angular configuration of the telescopes and their distance from the target.

EXPADES satisfies the previously mentioned requisites for studies with lowenergy light RIBs and has the additional advantages of compactness and portability. The components of the array can be easily reconfigured to suit many experiments. Moreover, it can be used as an ancillary detection system with $\gamma$-ray and neutron arrays. 
The paper is organized as follows: Section 2 will provide a general overview of the tracking system, the new telescope array, the mechanical structure and cooling system of the telescopes and the reaction chamber that houses the experimental set-up. The readout electronics developed for the treatment of the detector signals will be described in Sections 3, 4 and 5. The newly developed Analog to Digital Converter (ADC) and Trigger Supervisor Board (TSB) will be presented in Section 6 and 7, respectively, while the main features of the data acquisition system will be highlighted in Section 8. The results of offline tests and the in-beam performance of the detectors will be covered in Section 9 and 10, respectively. Some concluding remarks will finally be made in Section 11.

\section{Description of the RIB tracking system and the detection array}

The event-by-event RIB tracking system and the detection array EXPADES, installed in the reaction chamber at the final focal plane of the EXOTIC 3 facility, are schematically displayed in Figure 1.

\subsection{RIB tracking system}

The two Parallel Plate Avalanche Counters (PPACs) of the tracking system, developed by INFN-Napoli, are position-sensitive, fast, high-transparency de- 


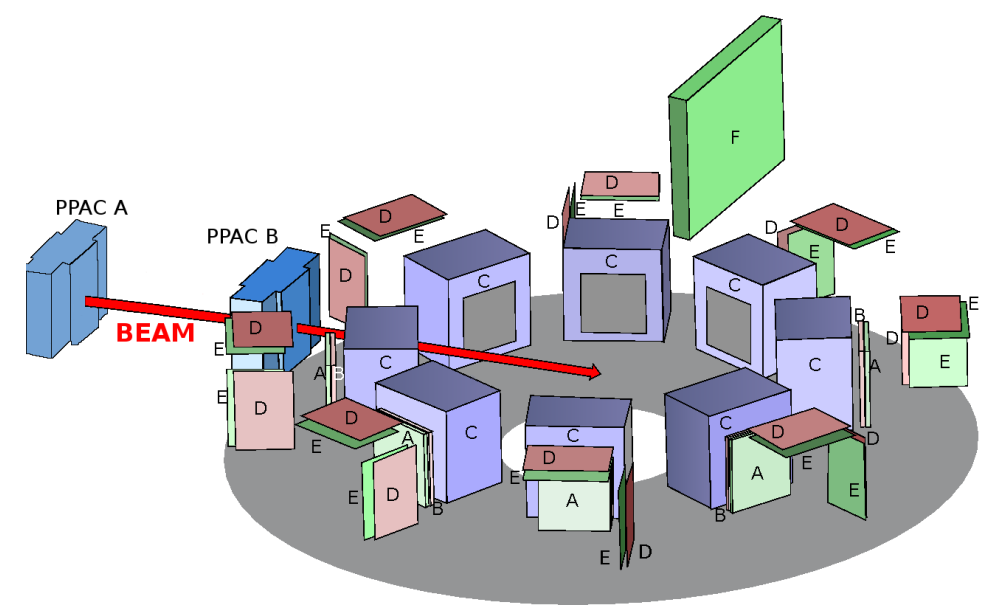

Figure 1. (color online) Schematic view of: a) the event-by-event tracking system of the RIB in-flight facility EXOTIC, consisting of two PPACs: PPAC A and PPAC $\mathrm{B}$, the second one being placed at the entrance of the reaction chamber; $\mathrm{b}$ ) the EXPADES array telescopes arranged in the reaction chamber. Each telescope is made up of: A) $300 \mu$ m-thick DSSSD ( $E_{\text {res }}$ stage); B) $40 \mu$ m-thick DSSSD $(\Delta E$ stage); C) Ionization chamber ( $\Delta E$ stage in experiments where the ions do not pass through the $40 / 60 \mu \mathrm{m}$ DSSSD stage); D) Low-noise charge-sensitive preamplifier boards for the $\Delta E$ DSSSDs; E) Electronic boards for the $E_{\text {res }}$ DSSSDs; F) Motherboard for the $E_{\text {res }}$ DSSSD electronics. The beam enters in the reaction chamber from the left passing through PPAC A and PPAC B.

137 tectors, radiation hard which can sustain counting rates up to $\sim 10^{6} \mathrm{~Hz}$. They ${ }_{138}$ are placed $909 \mathrm{~mm}(\mathrm{PPAC} \mathrm{A})$ and $365 \mathrm{~mm}$ (PPAC B) upstream the reaction target (see Figure 1). PPAC B is positioned at the entrance of the reaction 140 chamber.

${ }_{141}$ The PPAC has a three-electrode structure: a central cathode and two anodes, ${ }_{142}$ placed symmetrically with respect to the cathode at a distance of $2.4 \mathrm{~mm}$. The 
detector active area is $62 \times 62 \mathrm{~mm}^{2}$. The cathode is made of a $1.5 \mu$ m-thick stretched mylar foil with $30 \mathrm{~nm}$ of aluminum evaporated on both surfaces and it is mounted on a $0.8 \mathrm{~mm}$-thick fiberglass frame. Each anode is a mesh of 60 gold-plated tungsten $20 \mu \mathrm{m}$-thick wires in the $x$ and $y$ directions, with a spacing of $1 \mathrm{~mm}$. The wires of the first anode are oriented horizontally and those of the second one vertically. The position information of a particle crossing the PPAC is extracted from the anode signals by using a delay-line readout. Each wire is electrically connected to discrete LC circuit delay lines of $2.3 \mathrm{~ns} / \mathrm{mm}$ each, with a $50 \Omega$ impedance, resulting in a total delay of $138 \mathrm{~ns}$ in both the $x$ and the $y$ direction. The cathode signal is used as a reference time for TOF measurements and for trigger purposes. Figure 2 shows a schematic diagram of the PPAC electrodes and photographs of the complete detector assembly.

\footnotetext{
The PPAC vessel is made of polycarbonate while the two windows are made of $1.5 \mu \mathrm{m}$-thick mylar foil each, glued on a $1.6 \mathrm{~mm}$-thick fiberglass frame. The mylar foil is supported by an aluminum frame on which stretched aramid (a class of heat-resistant and strong synthetic fibers) wires (0.2 mm-diameter) are mounted in $\mathrm{x}$ and $\mathrm{y}$ direction, resulting in an overall geometrical transparency of $95 \%$ (97.5\% is the geometrical transparency for each window). The vacuum seal is obtained by means of a 1 mm-thick silicone rubber frame sandwiched between the fiberglass frame where the mylar foil is glued and the PPAC vessel.
} 


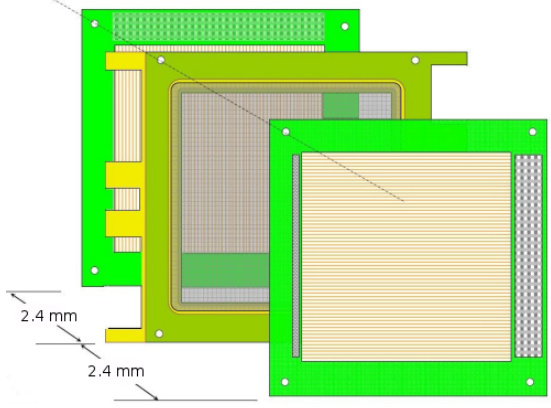

The PPAC is filled with isobutane $\left(\mathrm{C}_{4} \mathrm{H}_{10}\right)$ at a working pressure of $10-20$ mbar. Isobutane has a high gain enabling operation at low pressures [33]. The cathode is biased at a negative potential of 550-970 V, depending on the chosen pressure, while the anodes are kept at ground potential. In this way, reduced electric fields of $200-230 \mathrm{~V} / \mathrm{cm} / \mathrm{mbar}$ are obtained. The gas is continuously flowed to avoid contamination due to outgassing from the detector surfaces. An automatic control system, manufactured by the Bronkhorst High-Tech [34], is used to ensure constant gas flow and pressure in the detector with a stability better than $1 \%$ during the run.

Figure 2. (color online) Left panel: Schematic exploded view of the PPAC's electrodes. Middle panel: Electrode package. Right panel: Final assembly of the PPAC.

\subsection{EXPADES}
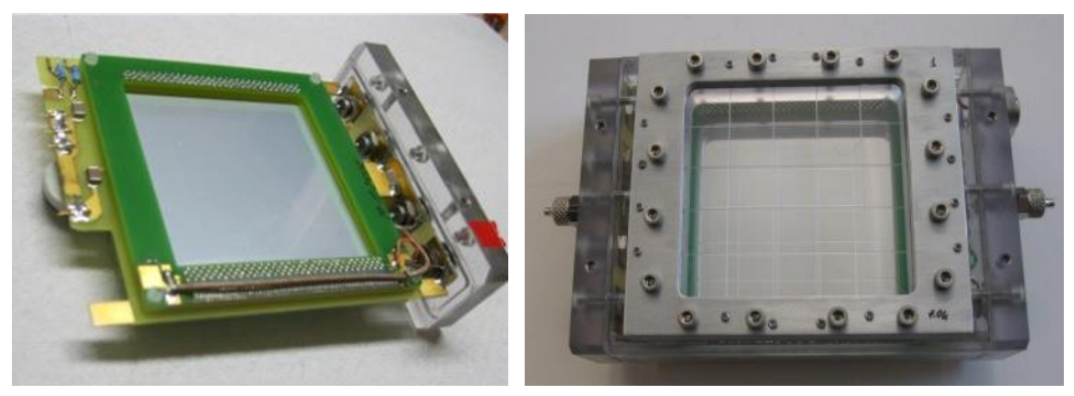

EXPADES is an array of eight telescopes arranged in a cylindrical configu- 


\subsubsection{DSSSD}

We use $40 / 60 \mu \mathrm{m}$-thick DSSSDs for the $\Delta E$ stage (elements B in Figure 1), whereas we adopt $300 \mu \mathrm{m}$-thick DSSSDs for the $E_{\text {res }}$ layer (elements A in Figure 1), manufactured by Micron Semiconductor Ltd. (BB7(DS)-40/60 and BB7(DS)-300, respectively) [35]. Each DSSSD has 32 junction and 32 ohmic elements (strips). The strips are $64-\mathrm{mm}$ long, with $2 \mathrm{~mm}$ pitch size and 40 $\mu \mathrm{m}$ inter-strip separation. The junction strips of the front $(y)$ side are oriented orthogonally to the ohmic strips of the back $(x)$ side, defining thus a $\sim 2 \times 2 \mathrm{~mm}^{2}$ pixel structure. For experiments requiring the detection of more energetic particles than those stopped in the $E_{\text {res }}$ layer, few $1 \mathrm{~mm}$-thick DSSSDs were recently purchased, to substitute the $300 \mu$ m-thick DSSSDs or to be used in addition to the previous stages.

The choice of the electronic front end of the DSSSDs was based on a compromise between the requirement for high granularity, good energy and good time resolution and that to maintain low the overall cost. Application Specific Integrated Circuit (ASIC)-based electronics was employed for the treatment of the $E_{\text {res }}$ signals (see Section 4). ASIC electronics allows us to handle 32 energy signals of each side of the $300 \mu \mathrm{m}$-thick $E_{\text {res }}$ DSSSD, ensuring a high granularity with a very low cost at the expense, however, of the possibility to 
perform TOF measurements with the requested time resolution (due to the lack of a constant fraction discriminator in the chip, see Section 4.1). Moreover, the chosen ASIC chip being optimized for capacitance up to about 70 $\mathrm{pF}$, could not be used for the much higher capacitance strips of the 40/60 $\mu \mathrm{m}$ DSSSD $\Delta E$ stage. For the signal readout of these detectors a compact low-noise electronics with an adequate dynamic range for the considered experiments ( $\sim 100 \mathrm{MeV}$ full range) and good energy and timing characteristics was developed (see Section 3.2 and Section 3.3). To reduce the cost of the $\Delta E$ stage custom electronics, lower than that of commercially available electronics but still much higher than the ASIC one, the 32 strips of each DSSSD side were reduced to 16 by short-circuiting two-by-two adjacent strips.

As can be seen in Figure 1 the charge-sensitive preamplifiers for the $\Delta E$ DSSSDs (element D in the figure) as well as the boards containing the ASIC electronics (elements $\mathrm{E}$ in the figure) for the $E_{\text {res }}$ DSSSDs are placed under vacuum in the proximity of the array. This was done mainly for three reasons:

(1) to have a compact set-up (detectors + electronics);

(2) to minimize the internal and external connections and

(3) to overcome the environmental noise at the EXOTIC beamline. In this way, we manage to keep as low as possible the DSSSDs electronic thresholds, typically 300-500 keV. 
In some experiments, the unambiguous identification by means of the $\Delta E$ $E_{\text {res }}$ technique of reaction products with range in silicon shorter than $40 / 60$ $\mu \mathrm{m}$ might be of crucial relevance. A valid alternative to allow for $\Delta E-E_{\text {res }}$ identification of all the considered ions, is the use of an IC that can be handled easily, presents thickness uniformity, possibility to tune the effective thickness by changing the gas pressure, offers the chance of a large detection surface and does not present radiation damage problems.

In our case, the construction of eight transverse-field ICs [36; 37; 38] was undertaken by INFN-Napoli. The choice of conventional transverse-field instead of axial-field (see for instance $[39 ; 40 ; 41]$ ) detectors was based on the following considerations: typically, an axial-field device presents a more uniform charge collection than a transverse-field IC, that shows some non-uniformities in the fringing fields near the entrance and exit windows, limiting thus its energy and charge resolution. However, axial field devices with a Frisch grid, suffer from the fact that some incident particles scatter off the grid contributing to the background of the telescope and limiting the transparency of the detector. Being the energy and charge resolution of the constructed transverse-field prototype enough for a good identification of the considered ions and comparable with that of an axial-field device (see discussion in Section 9.2 for $\alpha$ particles and in Section 10.2 .1 for $Z=8$ ions), this design was adopted for 239 the EXPADES array. The ICs (elements $\mathrm{C}$ in Figure 1) can be used as an 
alternative $\Delta E$ stage or to build up more complex triple telescopes.
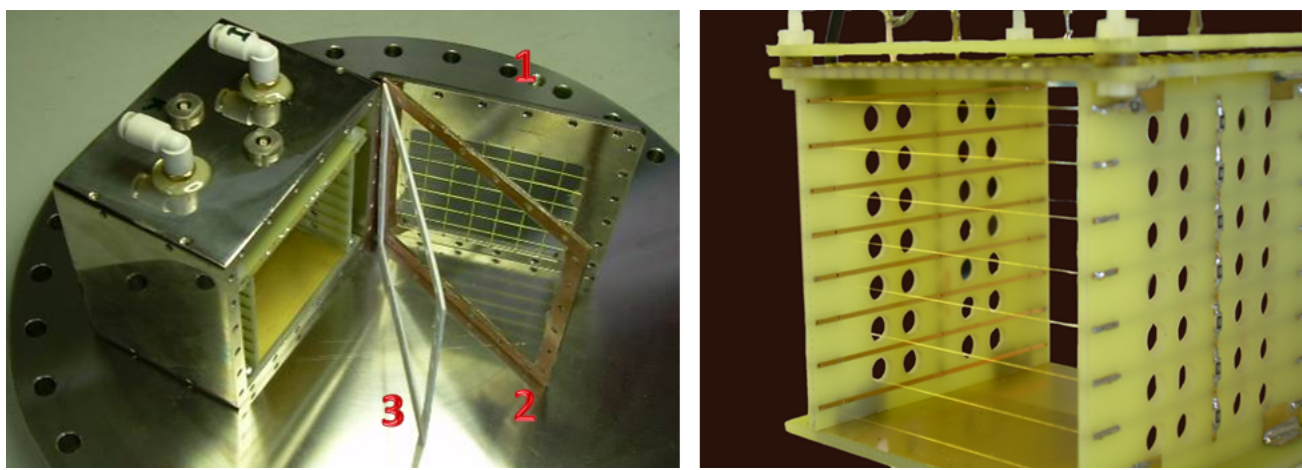

Figure 3. (color online) Left-hand side: IC and exploded view of the entrance window. Right-hand side: Electrodes, Frisch grid, field-shaping guard rings and field-shaping guard strips to ensure uniformity of the electric field.

Each IC is housed in a $100 \times 100 \times 68 \mathrm{~mm}^{3}$ chromium-plated brass vessel (see left panel of Figure 3). A $1.5 \mu$ m-thick mylar foil, glued on a 1.6 mm-thick fiberglass frame (element 2 in Figure 3, left panel), is used for the $65 \times 65 \mathrm{~mm}^{2}$ entrance and exit windows. The mylar foil is supported by a chromium-plated brass frame on which stretched nylon wires $(0.14 \mathrm{~mm}-$ diameter, $10 \mathrm{~mm}$-spacing) are mounted in both the $\mathrm{x}$ and $\mathrm{y}$ direction (element 1 in Figure 3, left panel). The wires define an overall geometrical transparency of about $95 \%$ ( $97.5 \%$ is the geometrical transparency of each window). The vacuum seal of the IC is obtained by means of a $1 \mathrm{~mm}$-thick teflon frame (element 3 in Figure 3, left panel) sandwiched between the fiberglass frame on which the mylar foil is glued and the IC vessel.

The IC active depth along the ion direction is $61.5 \mathrm{~mm}$. The active height, i.e. the distance between the cathode and the anode, is $68 \mathrm{~mm}, 64 \mathrm{~mm}$ being 
the distance between the cathode and the Frisch grid placed in-between (see right-hand side of Figure 3). The electrodes are made of $2 \mathrm{~mm}$-thick goldplated copper-coated $(40 \mu \mathrm{m})$ fiberglass frame and have a surface of $59 \times 61.5$ $\mathrm{mm}^{2}$. The Frisch grid is made of a $50 \mu \mathrm{m}$-thick gold-plated tungsten wire mesh with a $4 \mathrm{~mm}$-spacing (both in $\mathrm{x}$ and $\mathrm{y}$ direction). To have a uniform electric field in the active volume, 8 field-shaping guard rings (made of $50 \mu \mathrm{m}$-thick gold-plated tungsten wire) at a distance of $8 \mathrm{~mm}$ from each other are added while the field uniformity along the incident particle direction is maintained by gold-plated copper-coated strips (with $8 \mathrm{~mm}$ spacing) on fiberglass frame. The guard rings are connected to a voltage divider chain of 5.6 M $\Omega$ resistors. The cathode (anode) bias is $-300(+100)$ V, while the Frisch grid is at ground.

The IC is filled with carbon tetrafluoride $\left(\mathrm{CF}_{4}\right)$, chosen for its high electronic stopping power, because it can work with a relatively low gas pressure and for the high electron drift velocity [42]. The operational gas pressure can be varied up to 100 mbar, depending on the incident ion energy and on the species to be detected. The gas is transported in the internal part of the IC by a 6 mm-diameter tube, thus ensuring a good circulation everywhere. The gas is continuously flowed to avoid contamination due to outgassing from the detector surfaces. Also in this case we use the automatic control system manufactured by the Bronkhorst High-Tech [34].

274 To increase the IC anode signal to noise ratio, the employed charge-sensitive 25 preamplifier, described in Section 3.1, is mounted on the IC vessel (by using 
the two LEMO connectors, see left panel of Figure 3).

\subsection{Mechanical structure and cooling system of the telescopes}
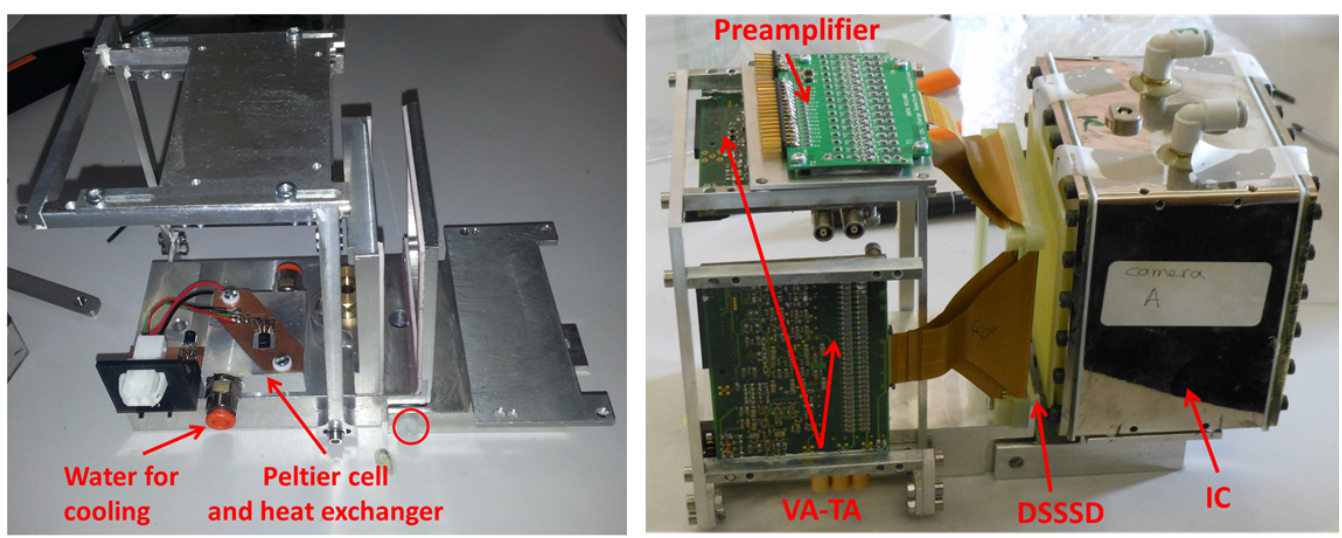

Figure 4. (color online) Left-hand side: Aluminum mechanical structure of a telescope, Peltier cell, heat exchanger and water-flow system for the cooling of the DSSSDs and of the electronic boards. The red circle indicates the plastic screw that isolates thermally the two parts of the mechanical structure: that of the IC and that of the DSSSDs. Right-hand side: a three-stage telescope, composed of an IC and two DSSSDs, and the DSSSD electronic boards. Preamplifier stands for the charge-sensitive preamplifier of the $\Delta E$ DSSSD stage and VA-TA stands for the electronic board of the $E_{\text {res }}$ stage (for details see Sections 3 and 4).

The telescopes of the EXPADES array and the associated electronics are mounted on an aluminum mechanical structure, shown in the left-hand side of Figure 4. The structure is cooled down to about $-20^{\circ} \mathrm{C}$ with the aid of to dissipate the heat produced by the electronics and to improve the detector 
$303(\sim 5 \%$ of $4 \pi \mathrm{sr})$.

performance. A plastic screw (red circle in Figure 4, left panel) isolates the aluminum structure of the IC from that of the DSSSDs. The picture in the right-hand side of Figure 4 displays a three-stage telescope, composed of an IC and two DSSSDs and the assembly of the DSSSD electronic boards.

The telescope structures are fixed on a plastic (Derlin) platform, to guarantee thermal isolation, and then on a rotating aluminum table as can be seen in Figure 5. In the original configuration of the detection array, the eight telescopes are located at the following mean polar angles (with respect to the beam direction): $\theta_{l a b}= \pm 27^{\circ}, \pm 69^{\circ}, \pm 111^{\circ}$ and $\pm 153^{\circ}$. However, different configurations can easily be achieved by properly turning the table to meet the requirements of the considered experiment. The distance of the detectors from the target can be varied continuously from a minimum value of $105 \mathrm{~mm}$ to a maximum of $225 \mathrm{~mm}$, which corresponds to an angular resolution for a pixel from $\Delta \theta=1^{\circ}$ to $0.5^{\circ}$. Figure 6 depicts the array solid angle coverage in the original configuration for five distances of the DSSSDs from the target and Table 1 summarizes the ranges of polar angles $\theta_{\text {lab }}$ spanned by each telescope. The maximum solid angle coverage (achieved in the configuration with only DSSSDs in use) is $2.72 \mathrm{sr}(\sim 22 \%$ of $4 \pi \mathrm{sr})$. When all eight ICs are employed, the DSSSDs have to be placed at a minimum distance of $225 \mathrm{~mm}$ from the target position and the maximum solid angle coverage decreases to $0.64 \mathrm{sr}$ 


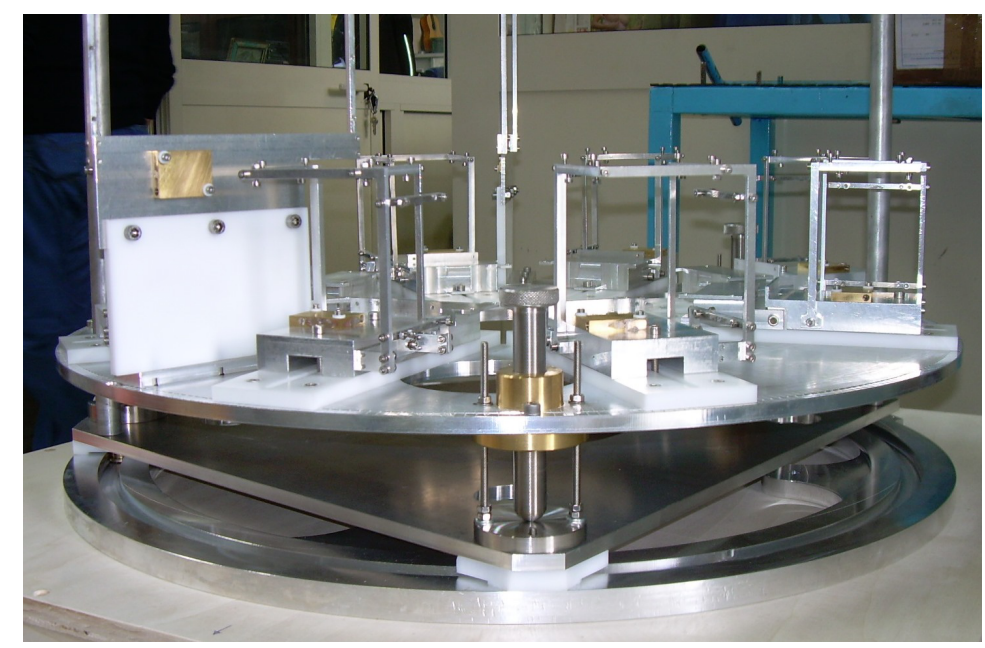

Figure 5. (color online) The supports of the telescopes are mounted on Derlin platforms (in white colour) placed on a rotating aluminum table. This table separates horizontally the reaction chamber in two volumes: the upper volume and the lower one (see also Figure 7 that illustrates a schematic view of the reaction chamber).

\subsection{Reaction chamber}

The reaction chamber, placed at the final focal plane of the EXOTIC facility, houses the PPAC $\mathrm{B}$ and the detection array EXPADES. It is a $778 \mathrm{~mm}$ diameter, $4 \mathrm{~mm}$-thick stainless steel cylindrical chamber that was designed for an optimal use of EXPADES in different configurations. The presence of gas detectors (ICs and PPAC), working with different gases and at different pressures, required the use of an internal system for the distribution of gas so as to ensure the same flowing and the same pressure in all the detectors of the same type (groups of ICs or PPAC). Since the electronic boards and the DSSSDs are cooled with Peltier cells and heat exchangers which must extract heat by circulation of water, it was necessary to foresee a distribution 


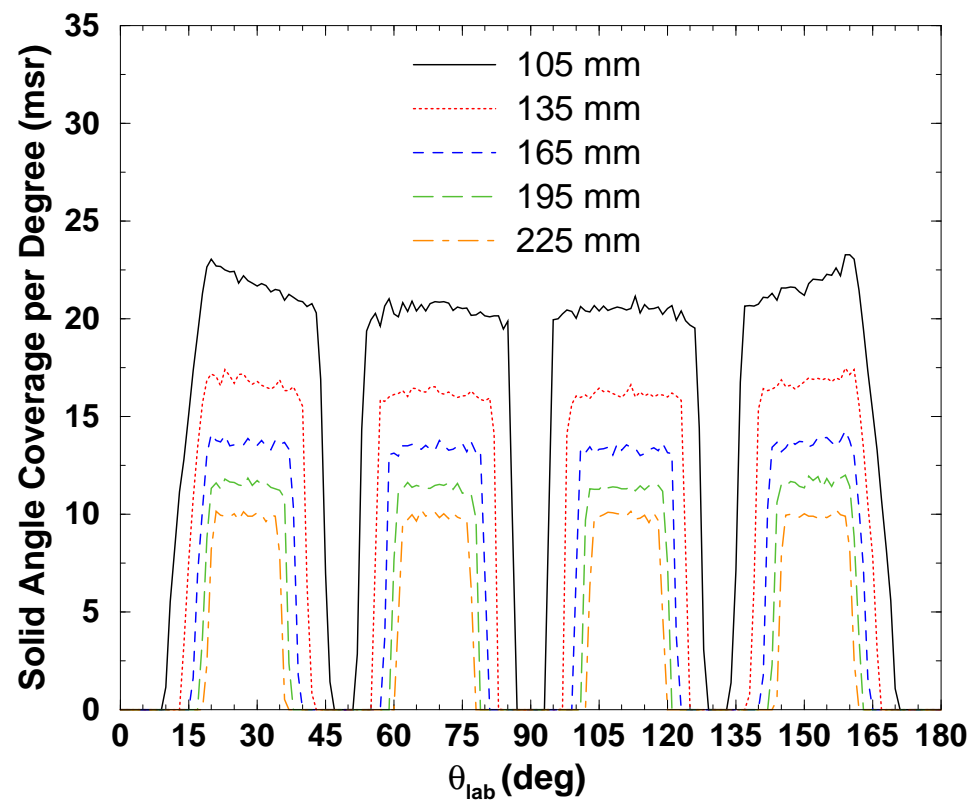

Figure 6. (color online) Solid angle coverage for five detector-target distances in the interval from 105 to $225 \mathrm{~mm}$ in the original configuration of the array. The reported values are the results of Monte-Carlo simulations for a point-like source.

system inside the reaction chamber for both the cooling liquid and for the required electrical power necessary for the Peltier cell operation. For the above 
backing and sensor systems for the activation of the pneumatic valves) and for handling the target ladder system. The target ladder has five positions, to fix up to four targets and a silicon detector for monitoring purposes.

To allow the realization of experiments with RIBs impinging on both solid and gas reaction targets, a small chamber housing the PPAC B was built. When requested, this small chamber isolates, through a $2 \mu$ m-thick Havar window, the two PPACs and the beam line (held at vacuum) from the reaction chamber that is filled with gas at pressures ranging from 0.4 to 1 bar. In this case, the reaction between the RIB and the gas target can occur at any point along the RIB trajectory inside the reaction chamber.

The mechanical supports of the detectors, the PPAC B chamber and the 7 reaction chamber were designed and built at INFN-Napoli.

The complexity of the whole experimental set-up, including ten gas detectors, eight ICs and two PPACs along the EXOTIC beamline, operating with different gases at different pressures, required the design and the construction (by INFN-LNL and INFN-Napoli) of an automatic control system to perform safely operations of venting, vacuum and flowing gas in the detectors avoiding user mistakes and/or preventing hardware failures which could cause serious damage to the system such as the breaking of thin mylar windows and of the wire electrodes. The control system is based on the CJ1M-CPU13 Programmable Logic Controller (PLC), supplied by OMRON, and an archi- 
tecture that allows to control the system both locally and remotely. The PLC is equipped with several I/O units and a board for direct link with the PLC of vacuum and flow control system.

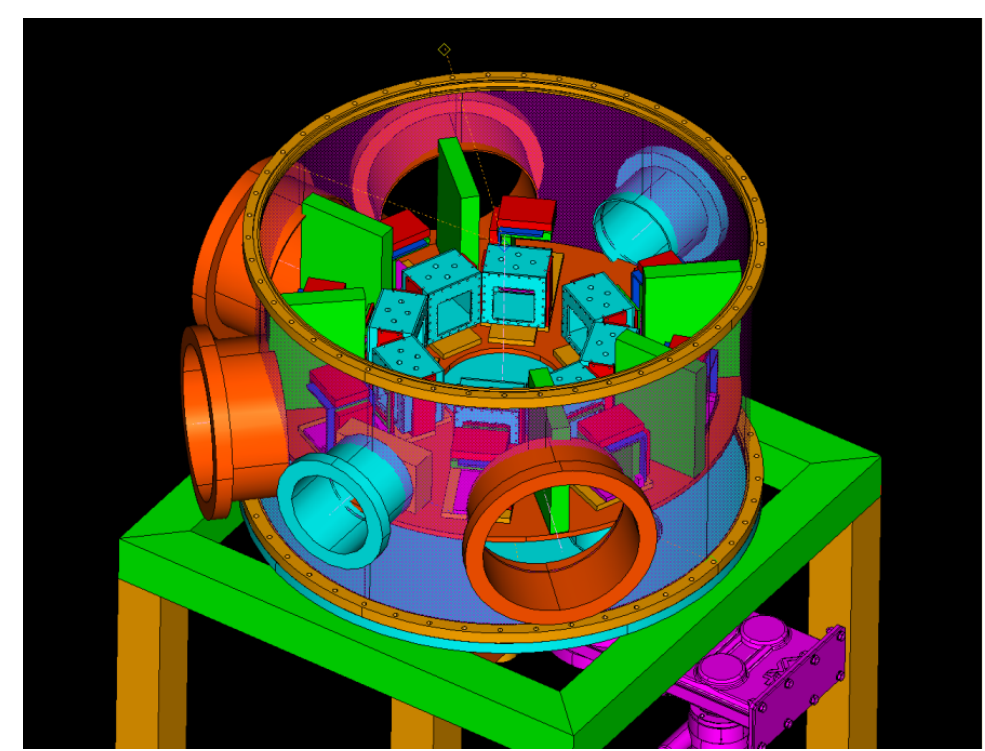

Figure 7. (color online) Schematic view of the reaction chamber installed at the final focal plane of the EXOTIC facility.

\section{$3 \quad \Delta E$ readout electronics}

\subsection{IC low-noise charge-sensitive preamplifier}

The main features of the preamplifier are the following: 
Table 1

(color online) Ranges of polar angles $\theta_{l a b}$ spanned by the telescopes of the array for five DSSSD-target distances in the interval from $105 \mathrm{~mm}$ to $225 \mathrm{~mm}$. The last entry indicates the overall solid angle coverage of the array at the corresponding distance. The reported values are the results of Monte-Carlo simulations for a pointlike source.

\begin{tabular}{|l|c|c|c|c|c|}
\hline d (mm) & 105 & 135 & 165 & 195 & 225 \\
\hline Tel. 1 & {$\left[13^{\circ}, 44^{\circ}\right]$} & {$\left[15^{\circ}, 41^{\circ}\right]$} & {$\left[17^{\circ}, 38^{\circ}\right]$} & {$\left[19^{\circ}, 36^{\circ}\right]$} & {$\left[20^{\circ}, 35^{\circ}\right]$} \\
Tel. 2 & {$\left[53^{\circ}, 86^{\circ}\right]$} & {$\left[56^{\circ}, 82^{\circ}\right]$} & {$\left[59^{\circ}, 80^{\circ}\right]$} & {$\left[60^{\circ}, 78^{\circ}\right]$} & {$\left[61^{\circ}, 77^{\circ}\right]$} \\
Tel. 3 & {$\left[94^{\circ}, 127^{\circ}\right]$} & {$\left[98^{\circ}, 124^{\circ}\right]$} & {$\left[100^{\circ}, 121^{\circ}\right]$} & {$\left[102^{\circ}, 120^{\circ}\right]$} & {$\left[103^{\circ}, 119^{\circ}\right]$} \\
Tel. 4 & {$\left[136^{\circ}, 167^{\circ}\right]$} & {$\left[139^{\circ}, 165^{\circ}\right]$} & {$\left[142^{\circ}, 163^{\circ}\right]$} & {$\left[144^{\circ}, 161^{\circ}\right]$} & {$\left[145^{\circ}, 160^{\circ}\right]$} \\
\hline$\Delta \Omega(\mathrm{sr})$ & 2.72 & 1.70 & 1.16 & 0.84 & 0.64 \\
\hline
\end{tabular}

361 slope;

362

363

364

- Energy sensitivity for silicon detector: $90 \mathrm{mV} / \mathrm{MeV}$;

- Output Voltage: $8 \mathrm{~V} \max (4 \mathrm{~V}$ on $50 \Omega$ termination);

- Decay Time: $600 \mu$ s;

- FWHM noise measured at $3 \mu$ s shaping time: $<1.5 \mathrm{keV}(0 \mathrm{pF}) 12 \mathrm{eV} / \mathrm{pF}$

- HV to input resistance: $100 \mathrm{M} \Omega$;

- Max HV input: $200 \mathrm{~V}$;

- Test capacitance: $1 \mathrm{pF}$; 
- Power consumption: $<250 \mathrm{~mW}$.

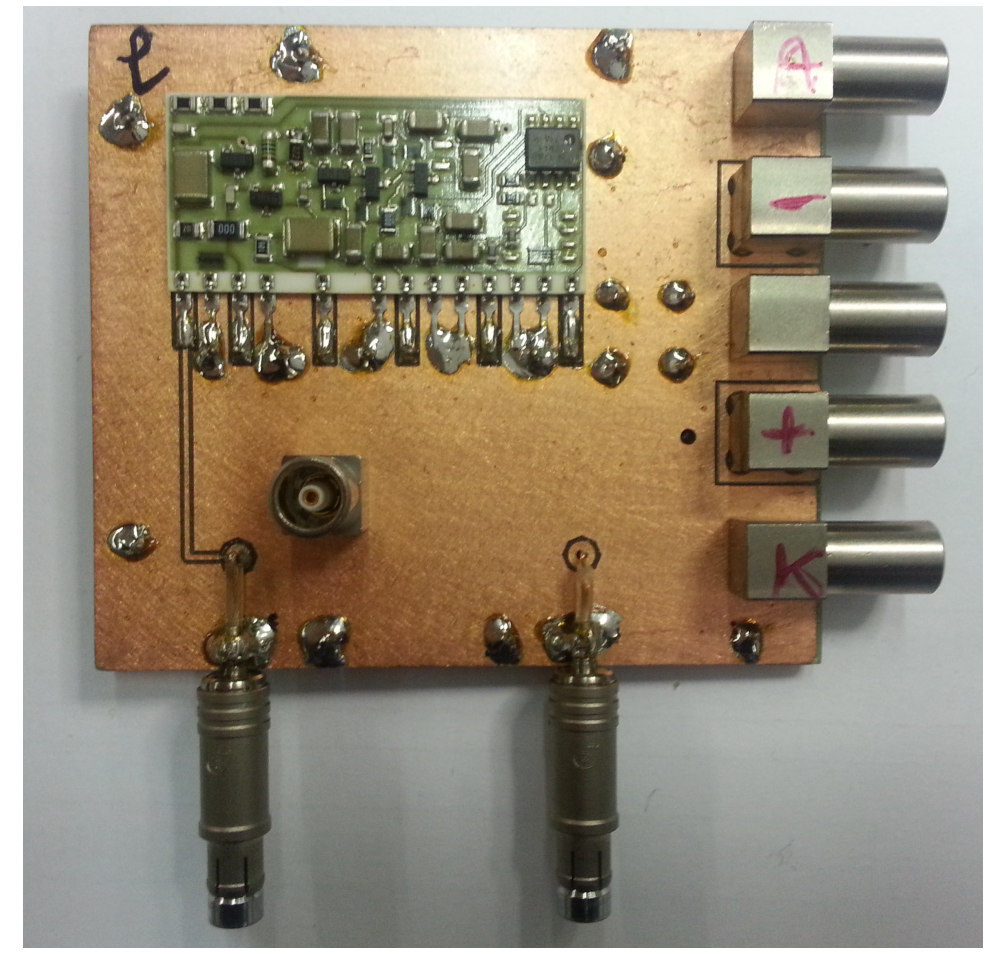

Figure 8. (color online) PCB of the IC charge-sensitive preamplifier that is mounted on the IC vessel.

\subsection{DSSSD low-noise charge-sensitive preamplifier}

367

A 16-channel custom low-noise ac-coupled charge-sensitive preamplifier (Figure 9) was specifically designed by INFN-Milano to match the high capacitance of the large-area $40 / 60 \mu \mathrm{m}$-thick DSSSDs, used as $\Delta E$ stage. The main features of the preamplifier are the following:

- Number of channels: 16 ;

- Board size: $78 \mathrm{~mm} \times 47 \mathrm{~mm}$;

- FWHM noise measured at $3 \mu$ s shaping time: $<3.3 \mathrm{keV}$ at $0 \mathrm{pF} ; 18 \mathrm{eV} / \mathrm{pF}$ 
slope;

375

376

377

378

379 $389 \quad 12 \mathrm{pF}$.

- Rise Time: $<3.3 \mathrm{~ns}$ at $0 \mathrm{pF} ; 28 \mathrm{~ns}$ at $600 \mathrm{pF}$;

- Sensitivity for silicon detector: $45 \mathrm{mV} / \mathrm{MeV}$;

- Pseudo Differential Output;

- Power Consumption: $<900 \mathrm{~mW}$.

The detector front and back sides are connected to the electronic boards with Kapton cables that are flexible and properly designed with low capacitance and ground shielding. These cables are obtained with a 4-layer (25 $\mu$ m-thick each) Kapton circuit, using galvanic gold deposition with no nickel backing, have a reduced length $(54 \mathrm{~mm})$ to render compact the array and a direct connection (without cable connectors) to the detector-chip interface card. Moreover, the signal lines (0.5 mm-pitch) are shielded both internally (separated each other with ground lines) and externally to reduce noise and cross talk between adjacent strips. To lower the capacity of this cable the ground shielding was not build as unique plan but as a grid. The capacity of the Kapton cable is about 391 on the preamplifier PCB. As mentioned previously, to maintain low the cost 392 of the $\Delta E$ readout electronics, the 32 strips of each DSSSD side were reduced 393 to 16 by short-circuiting two-by-two adjacent strips. 


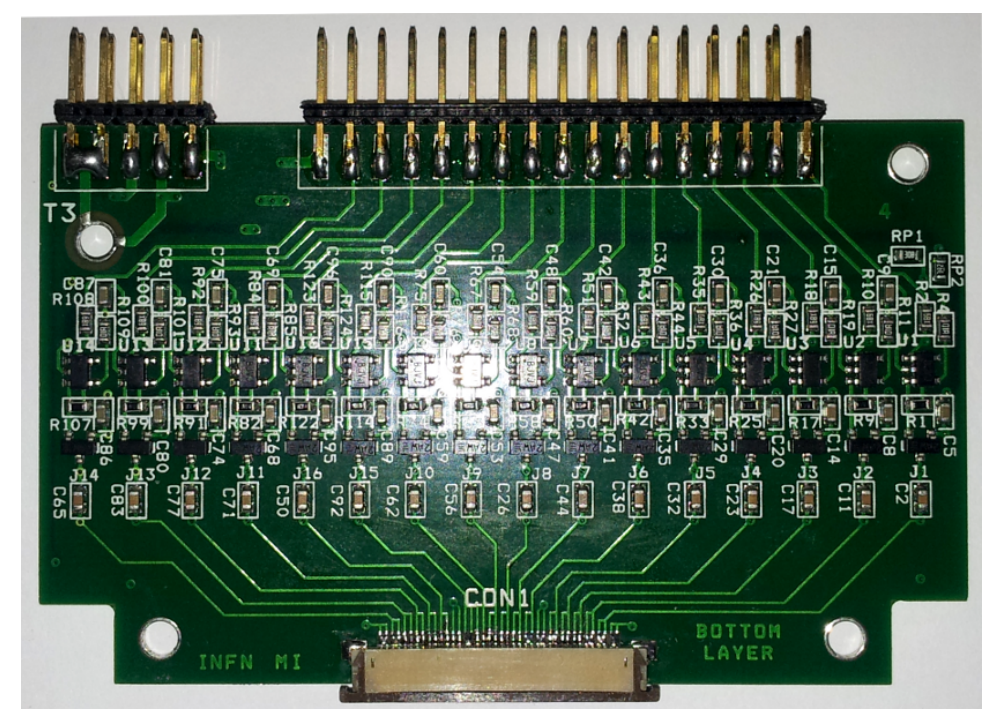

Figure 9. (color online) PCB housing 16 charge-sensitive preamplifiers for the electronic readout of signals originating from one side of the $40 / 60 \mu$ m-thick DSSSD detector (16 channels/side). The ERNI 36-pin connector, where the input Kapton cable is inserted into the PCB, is located on the PCB bottom part. The board power supply connector (left) and the output signal connector (center) are placed on the PCB upper part. 
Figure 10. (color online) Schematic representation of the connection of a DSSSD to the charge-sensitive preamplifiers of the front and back sides.

to a preamplifier on both sides (front and back) as schematically shown in Figure 10. Thus, in the DSSSD case, the reference of the preamplifier input of one detector side is the virtual ground of the preamplifier of the other detector side and not its own ground.

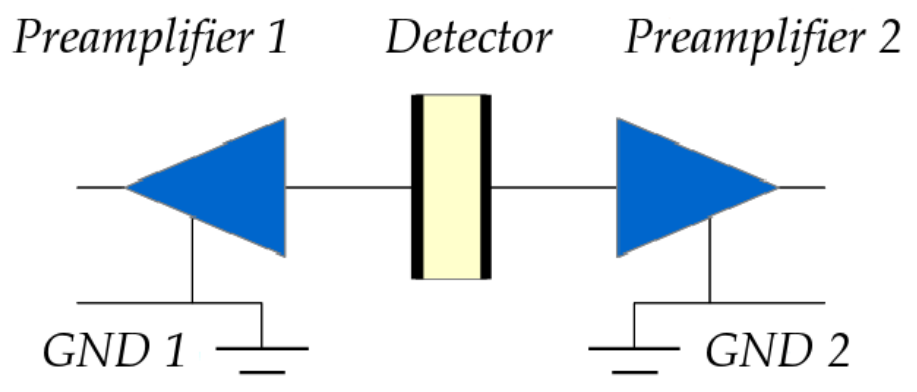

In these conditions, the noise on the ground of one preamplifier, due to the reflected signals, induces a signal at the input of the other preamplifier, causing instability and oscillations for both preamplifier signals. To overcome this problem, a 32-channel differential driver board (see left-hand side of Figure 11) was developed by INFN-Napoli. This board is placed immediately next to the preamplifier outputs and it is firmly connected to their grounds. The board receives the signals from the DSSSD front- and back-side preamplifiers, transforms them into true differential outputs and drives cables with a characteristic impedance of $110 \Omega$.

To reduce the number of connections, in the driver board were added two drivers of opposite polarity for the test signal sent to the preamplifiers and 

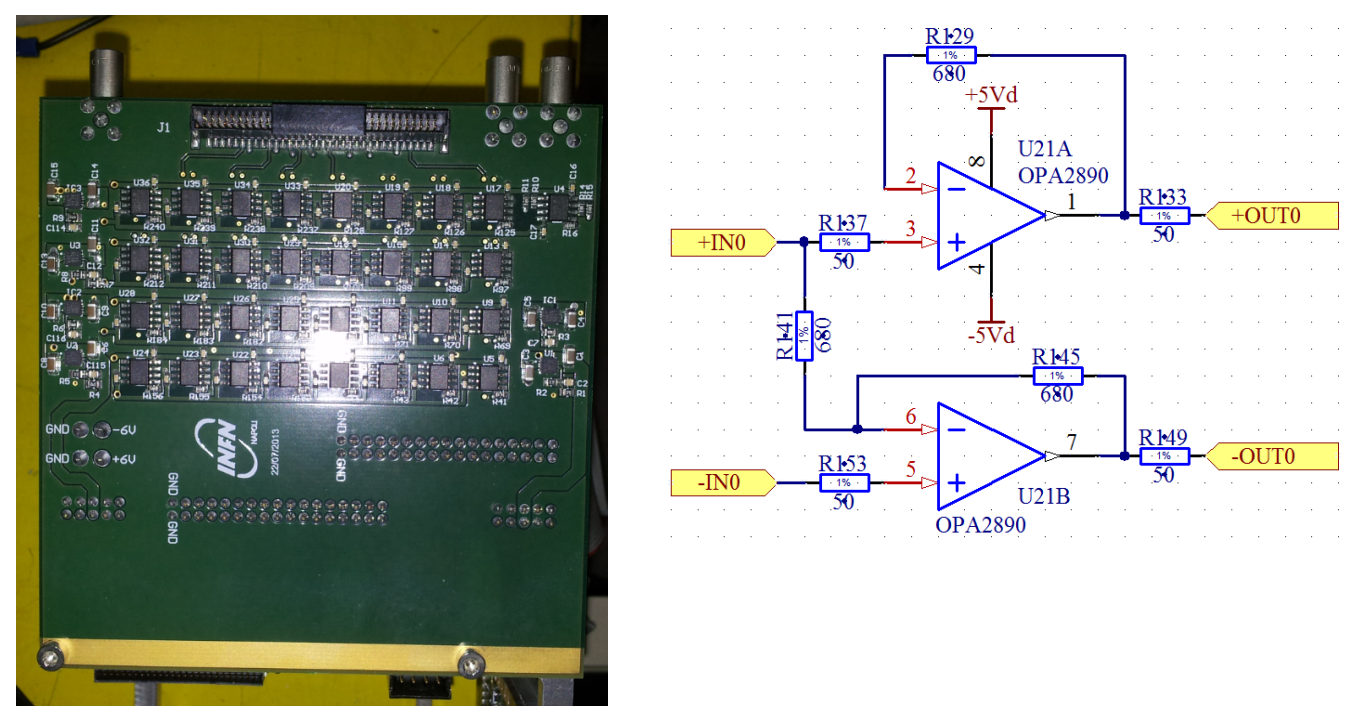

Figure 11. (color online) Left-hand side: PCB of the driver board for the DSSSD charge-sensitive preamplifiers. Right-hand side: schematic diagram of a single channel.

power supply low-noise regulators for each preamplifier board to prevent low-

423 in the driver board.

424 
hand side of Figure 11 we display the schematic diagram of a single channel.

\subsection{MEGAMP}

The 16 differential output signals coming from the preamplifier driver board and the outputs of the IC preamplifiers are processed by a specifically designed (by INFN-Milano) amplifier module called MEGAMP [44]. This module provides all the major information required by typical nuclear physics experiments: Energy, Timing and Pulse Shape Analysis. The MEGAMP is a single NIM module where 16 channels are housed. Each channel consists of two main sections related to energy and time parameters. The energy section consists essentially of a spectroscopy amplifier that accepts differential input signals. A linear gate and a stretcher section provides peak detection and hold during the readout sequence. The timing section provides both Time and Pulse Shape information. It is composed of two Constant Fraction Discriminators (CFDs) that are set to give an output signal at $30 \%$ and $80 \%$ of the signal leading edge and a Time to Amplitude Converter (TAC) circuit. An important feature of the module is the possibility to have a sequential readout of both energy and time information by means of a fast multiplexer circuit. With few external logic commands originating from the custom ADC (described in Section $6)$, the 32 (16 Energy +16 TAC) analog parameters can be readily acquired reducing the complexity and the cost of the acquisition system. The two-CFD part of the MEGAMP module is based on an older project [45] developed for 
the pulse shape discrimination technique with the detector CHIMERA (LNS-

INFN, Italy) in an incident energy regime of 20-30 MeV/nucleon [46]. The new elements of the MEGAMP module, specifically designed for our collaboration, concern the readout from the custom ADC, the peak-and-hold and TAC circuits and the Multiplexer circuit for the ADC.
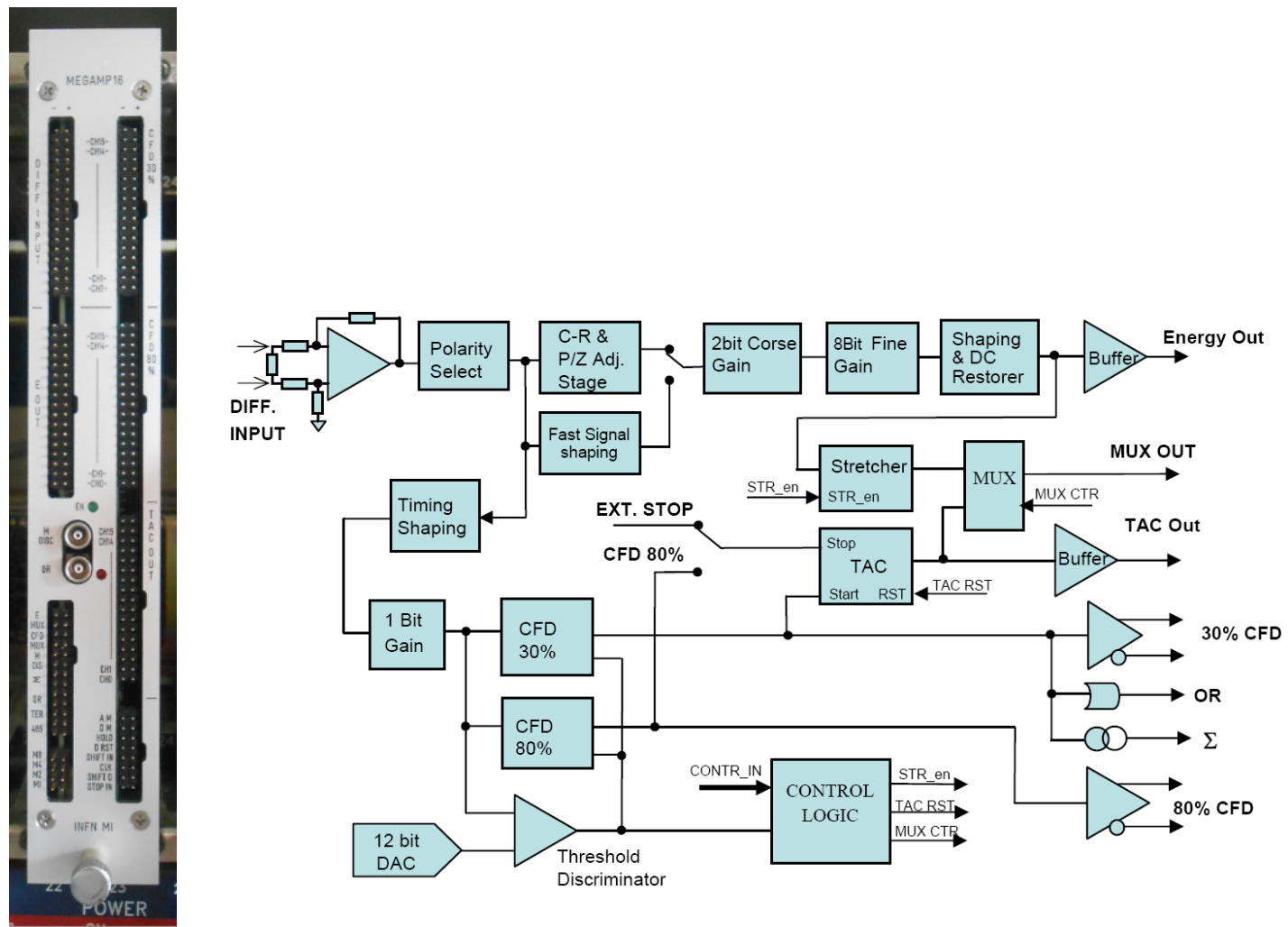

Figure 12. (color online) MEGAMP (left) and block diagram of a single channel of the MEGAMP (right).

Figure 12 shows a MEGAMP and the block diagram of one channel of the module. The first stage of the amplifier circuit is a differential receiver. It transforms the differential input signal into a single ended one. The high comsignal avoiding pickup noise problems. The following stage is the input polar- 
ity selection. The user must select the inversion or non-inversion of this stage in order to send the right signal polarity to the following Energy and Timing stage.

The Timing stage is composed of a circuit for the time shaping and of a fast amplifier with two selectable gains. The shaped signal then reaches the discriminator circuits. A comparator with a threshold set by a 12-bit Digital to Analog Converter (DAC) gives the enable signal to the rest of the logic circuits when the input signal exceeds the threshold value. The $30 \%$ CFD is used to provide a good timing information. The amplitude ratio is set to about $30 \%$ and the shaping delay can be selected according to the type of the detector signal. The $80 \%$ CFD is used for the pulse shape discrimination. It is set to about $80 \%$ of the amplitude ratio and the shaping delay is optimized for obtaining maximum sensitivity to the variation of the signal leading edge. Both CFD circuits have an automatic walk compensation and their outputs are present in differential ECL logic on the module front panel. The $30 \%$ CFD output gives the Start signal to the TAC circuit while the Stop signal can be hardware selected between the internal $80 \%$ CFD output and an external Common Stop. When the TAC stop signal is the $80 \%$ CFD output, the TAC gives a pulse shape information by means of the input signal rise time measurement. Figure 13 illustrates the working principle of the MEGAMP time analysis unit for obtaining the pulse shape information. When the TAC is set to the external Common Stop modality, it can be used for TOF mea- 
surements. The TAC reset control is generated from a control logic enabled from the threshold discriminator. The $30 \%$ CFD output is also involved in the logic OR and Multiplicity of the module. We notice here, that the pulse shape discrimination technique is useful mainly for incident energies higher than those of the EXOTIC RIBs due to the high thresholds above which such a discrimination becomes possible. As can be seen in [45], charge identification for ions with atomic number up to $Z=12$ was achieved with energy threshold ranging from $4.5 \mathrm{MeV} /$ nucleon $(Z=6)$ to $6.5 \mathrm{MeV} /$ nucleon $(Z=10)$. Although at low incident energies we cannot fully exploit this feature, at higher energies the pulse shape technique becomes a powerful method to discriminate ions that stop in the DSSSD $\Delta E$ stage.

The Energy Stage starts with two circuits able to handle the input signals coming from different types of detectors. The first circuit, optimized for signals coming from charge-sensitive preamplifiers, is composed of a set of two CR circuits (with time constant of 0.5 and $3 \mu \mathrm{s}$ ) giving the signal derivative with a pole zero compensation. The second circuit, for fast unipolar signals (like those originating from $\mathrm{BaF}_{2}$ scintillators, PPACs, etc), consists essentially of an approximate integrator with a time constant of $0.5 \mu$ s without pole zero compensation. Either circuit can be remotely selected by the user and connected to the amplifier section composed of a 2-bit coarse gain stage and a 8-bit fine gain stage. The amplified signal is sent to a shaping circuit with two selectable time constants $(0.5 \mu \mathrm{s}, 3 \mu \mathrm{s})$. At the end, a DC restorer stabilizes 

Gain 1 to $4 \mathrm{X}$; $0.02 \%$

the output dc level and reduces low-frequency fluctuations. In order to have a sequential readout, a stretcher circuit is also present. A Control Logic circuit enables the stretcher to capture the peak of the Gaussian output signal and hold it during the entire period of the readout. The stretcher and the TAC output are alternately switched on a fast analog multiplexer output and can be read from a single ADC channel.

The main specifications and performance of the MEGAMP are the following:

- Differential input: $\pm 4 \mathrm{~V} \max$ and $120 \Omega$;

- Energy output: $8 \mathrm{~V} \max -50 \Omega$ back termination;

- TAC output: $8 \mathrm{~V} \max$ - $50 \Omega$ back termination;

- Spectroscopic amplifier Shaping time: $0.5 \mu \mathrm{s}, 3 \mu \mathrm{s}$;

- Long tail or Fast unipolar input signal selection;

- Pole zero compensation: 50 to $1000 \mu$ s with 8-bit resolution (only for long

- Spectroscopic amplifier Gain: 2-bit Coarse Gain 1, 4, 16, 64 X, 8-bit Fine

- Timing Amplifier Gain: 1, 4 X (1 bit);

- Equivalent input noise (FWHM): $<10 \mu \mathrm{V}$ at $3 \mu$ s shaping time;

- Integral energy non-linearity for 10-90\% of the Full Scale Range (FSR): \pm

- Minimum Stretcher amplitude output: $<4 \mathrm{mV}$;

- DC restore Counting Rate Stability (shift of the peak): $0.08 \%$ from 0.1 to 
$10 \mathrm{kHz}$;

526

527

528

529

536

$539 \quad$ NIM output;

540

541

542

543

544

545

546

- CFD FWHM jitter time: $<150$ ps $(-500 \mathrm{mV}$, rise time $10 \mathrm{~ns})$;

- CFD walk: $<350 \mathrm{ps}(-80 \mathrm{mV}$ to $-4 \mathrm{~V}, 10 \mathrm{~ns}$ rise time);

- CFD 30\% Delay Setting: 7, 15, 30, 100 ns;

- Integral TAC non-linearity for 10-90\% of FSR: $< \pm 0.05 \%$;

- TAC Range from 600 ns to $2 \mu \mathrm{s}$;

- TAC Stop from CFD 80\% or External Common Stop;

- CFD 30\% Multiplicity Output = $1 \mathrm{~mA}$ for each channel;

- CFD 30\% OR output: fast NIM (with a Logic circuit allowing to disable a specific channel);

- Fast Multiplexed Read-Out (to ADC): Energy Stretched, TAC Out, THR Disc (CFD 30\%);

- Max Frequency Multiplexer Read-Out: 5 MHz;

- Multiplexed monitor output: spectroscopic amplifier output and 30\% CFD

- All parameters programmable through an RS 485 serial interface;

- CFD Threshold 1 to $4096 \mathrm{mV}$ 12-bit resolution;

- OR LED, MUX ENABLE LED.

54 In summary, a MEGAMP module provides the following output signals:

- 16 differential inputs (34-pin $100 \mathrm{mil}$ (2.54 mm) pitch connectors);

- 16 Energy outputs (34-pin $100 \mathrm{mil}$ (2.54 mm) pitch connectors);

- 16 TAC outputs (34-pin 100 mil (2.54 mm) pitch connectors); 
- $1630 \%$ CFD ECL differential outputs (34-pin 100 mil (2.54 mm) pitch

548 connectors);

- 16 80\% CFD ECL differential outputs (34-pin 100 mil (2.54 mm) pitch $550 \quad$ connectors);

551

- 2 OR outputs: one NIM (lemo connector) and one ECL;

- 2 Discriminator Multiplicity outputs $\left(\mathrm{M}_{d i s c}\right)$ : one NIM (lemo connector) and 553 one ECL;

554

- 1 Monitor Energy Multiplexer output $\left(\mathrm{E}_{M U X}\right)$;

555

- 1 Monitor 30\% CFD Multiplexer output;

556

- 1 analog output $\Sigma$;

557

558

- 2 RS485 connections;

- connection to ADC (16-pin $100 \mathrm{mil}$ (2.54 mm) pitch connectors) for control and fast multiplexer.

A MICROCHIP PIC18F series microcontroller handles the MEGAMP module. The parameters for each channel, that can be controlled via a SPI interface 52 from the microcontroller, are the following: inversion of the input signal polarity, shaping time of the spectroscopy amplifier, pole zero adjustment, coarse gain, fine gain, fast amplifier shaping time, fast amplifier gain, CFD threshold. Common parameters for all the channels that can be controlled are: TAC full scale and multiplicity threshold.

The MEGAMP module is remotely controlled via the serial RS485 standard. Each module has an address that can be set with a front panel jumper. When 


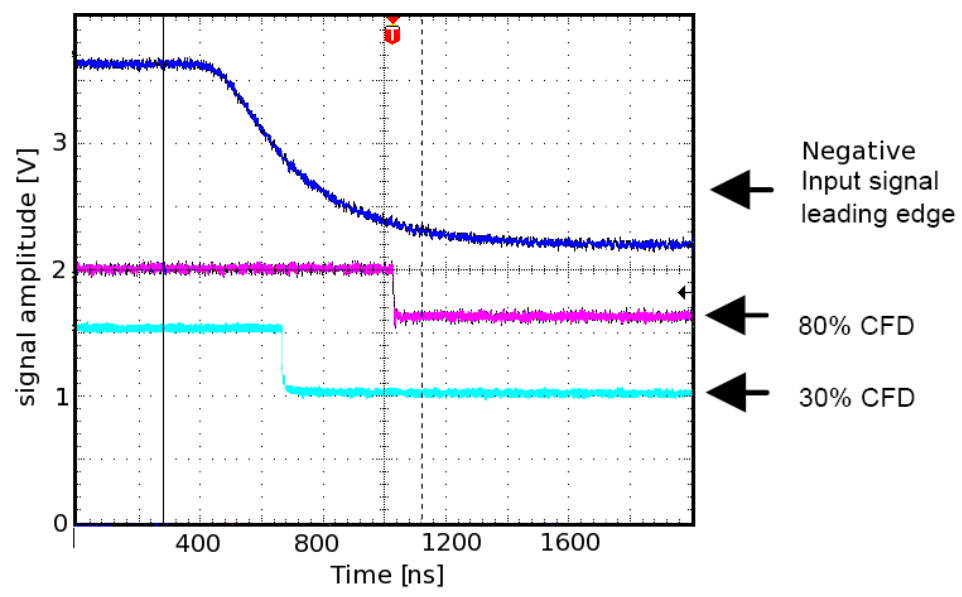

Figure 13. (color online) Example of the working principle of the MEGAMP time analysis section. The negative input signal is processed by two CFDs, respectively, at $30 \%$ and $80 \%$ of the signal leading-edge. The time difference between the outputs of the two CFDs is converted into an analog signal by an internal TAC. the MEGAMP module is turned on, the microcontroller restores the last setup values of the module reading the data stored in an internal EEPROM memory. To have a single RS485 control line, various MEGAMP modules are connected in daisy chain. The protocol employs only printed ascii characters allowing the use of a simple terminal emulator program to handle the whole chain of MEGAMP modules avoiding development of ad-hoc software.

To monitor the correct set-up of the channel parameters, two multiplexed monitor output signals are provided: one is the specroscopy amplifier output signal and the second one is the NIM $30 \%$ CFD output signal of the relative channel. When setting the parameters relative to a specific channel of a MEGAMP module, the microcontroller activates the multiplexer related to this channel. When a MEGAMP module is not selected, the two multiplexed 
594

595

596

${ }_{597} \cdot$ thickness: $725 \mu \mathrm{m}$

${ }_{598} \cdot$ power consumption: $3 \mathrm{~mW} /$ channel

${ }_{599} \quad \cdot$ shaping time: $1.9 \mu \mathrm{s}$

600 MEGAMP modules.

\section{$4 \quad E_{\text {res }}$ readout electronics} ergy signals, with the following specifications:

- technology: $0.35 \mu \mathrm{m}$ CMOS, epitaxial layer

. size: $4.4 \mathrm{~mm} \times 3.4 \mathrm{~mm}$

- gain: $150 \mu \mathrm{A} / \mathrm{pC}$

monitor outputs are in three state (high impedance). The above function allows a parallel connection of the multiplexed outputs of all the channels of all

As explained in Section 2.2.1, a completely different approach was followed in the design of the electronics for the DSSSD $E_{\text {res }}$ stage of the EXPADES telescopes. In this case the development of ASIC-based electronics, similar to the work described in Refs. [22; 47], was undertaken. The front and back DSSSD sides are connected to VA-TA boards (developed by INFN-Padova) through the already mentioned 54-mm long custom Kapton cables. Each VA-TA board houses two 32-channel chips, both manufactured by the company Gamma

- the linear chip VA32HDR14.2 (VA), for the analog treatment of the en- 
601 bias voltage: $\pm 2.5 \mathrm{~V}$

602

603 following specifications:

${ }_{604} \cdot$ technology: $0.8 \mu \mathrm{m} \mathrm{N}$-well CMOS, double-poly, double metal

605 size: $4.0 \mathrm{~mm} \times 3.4 \mathrm{~mm}$

$606 \quad \cdot$ thickness: $600 \mu \mathrm{m}$

$607 \cdot$ shaping time: $75 \mathrm{~ns}$

608 bias voltage: $\pm 2 \mathrm{~V}$

${ }_{609}$ The use of these two 32-channel chips allows an individual treatment of the 61032 strips of each detector side, thus achieving a position resolution of $\sim$ ${ }_{611} 2 \mathrm{~mm} \times 2 \mathrm{~mm}$ for the $E_{\text {res }}$ stage.

$612 \quad 4.1 \quad V A-T A$ board

61 614

615 616 617 618 620 619 signals (from top to bottom): detector bias (label "D"), VA test input signal

Figure 14 shows a picture of a VA-TA board. In the upper part of the figure, label "A" indicates the ERNI 36-pin connector (32 signal lines and 4 ground connections), where the Kapton cable is inserted into the board. The two 6 chips are located approximately at the center of the board and the letters 17 "B" and "C" label the chip VA and TA, respectively. On the left-hand side 618 of the VA-TA board we have three LEMO connectors used for the following (label "E") and TA trigger threshold signal (label "F"). Finally, in the lowest 

${ }_{623}$ (label "H").

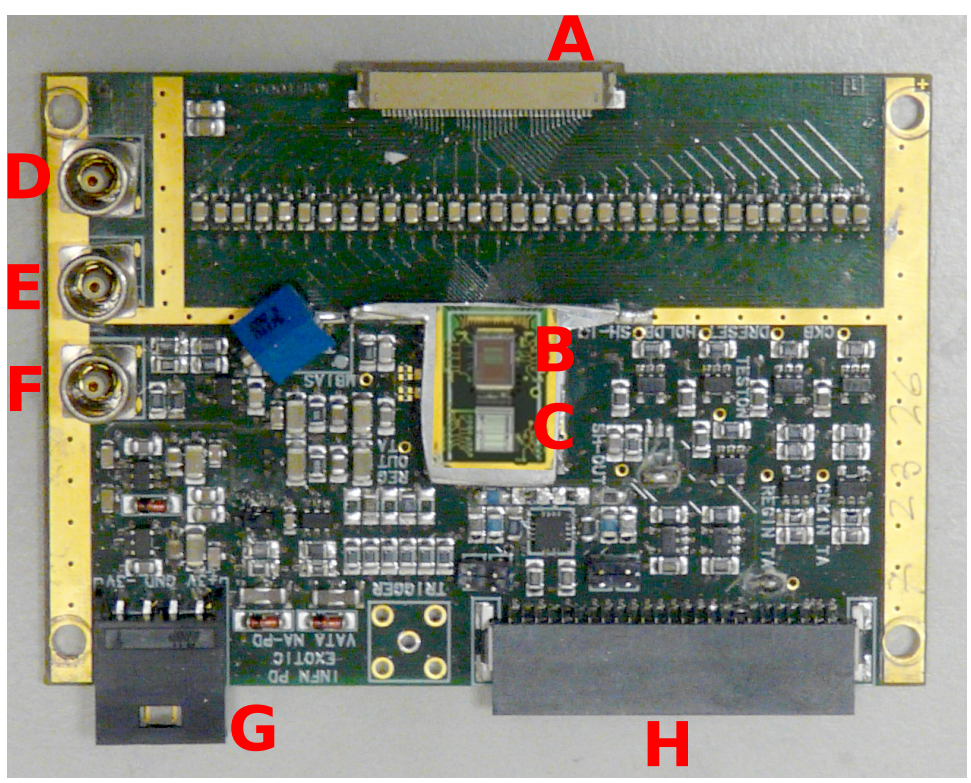

Figure 14. (color online) Image of a VA-TA board. The various labels indicate: A) ERNI 36-pin connector for the Kapton cable, B) VA chip, C) TA chip, D) LEMO connector for the detector bias, E) LEMO connector for VA test input signal, F) LEMO connector for the TA trigger threshold signal, $\mathbf{G}$ ) 4-pin connector for the VA-TA power supply and $\mathbf{H}$ ) 48-pin communication port with the motherboard.

The LEMO connector "D" is connected, through an output flange, to an external NIM power supply module. Connectors "E", "F", "G" and the port "H" of each VA-TA board are instead connected to a motherboard, also located under vacuum inside the reaction chamber. Figure 15 shows an example of connection between one DSSSD with two VA-TA boards and a motherboard. One motherboard can handle up to 8 VA-TA boards (i.e. $4 E_{\text {res }}$ detectors), 


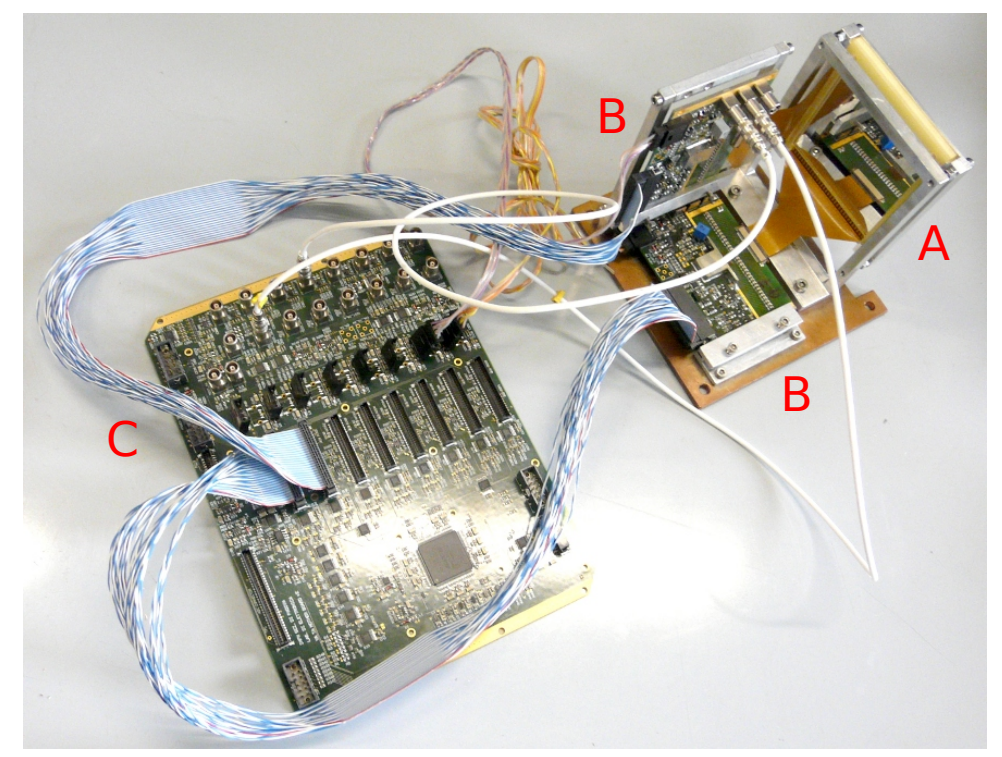

Figure 15. (color online) Example of connection between one detector (label "A"), two VA-TA boards (labels "B") and one motherboard (label "C").

thus heavily reducing the number of connections from inside to outside the reaction chamber.

Figure 16 illustrates the block diagram of a single channel of the VA-TA board. The chip VA essentially consists of a charge-sensitive preamplifier followed by a slow amplifier ( $2 \mu$ s peaking time) and by a sample-and-hold circuit. The amplification gain can be set, by the configuration of four jumpers on the VA-TA board itself, to match approximately the following full-scale ranges: 30, 52, 90 and $113 \mathrm{MeV}$. The chip TA schematically consists of a fast shaper (75 ns peaking time) and a leading-edge discriminator.

Figure 17 shows the generation of the output data stream for a single detector strip. Panel (a) displays the Fast Shaper output signal (see Figure 16) together with the threshold for the TA leading-edge discriminator (horizontal red line). 


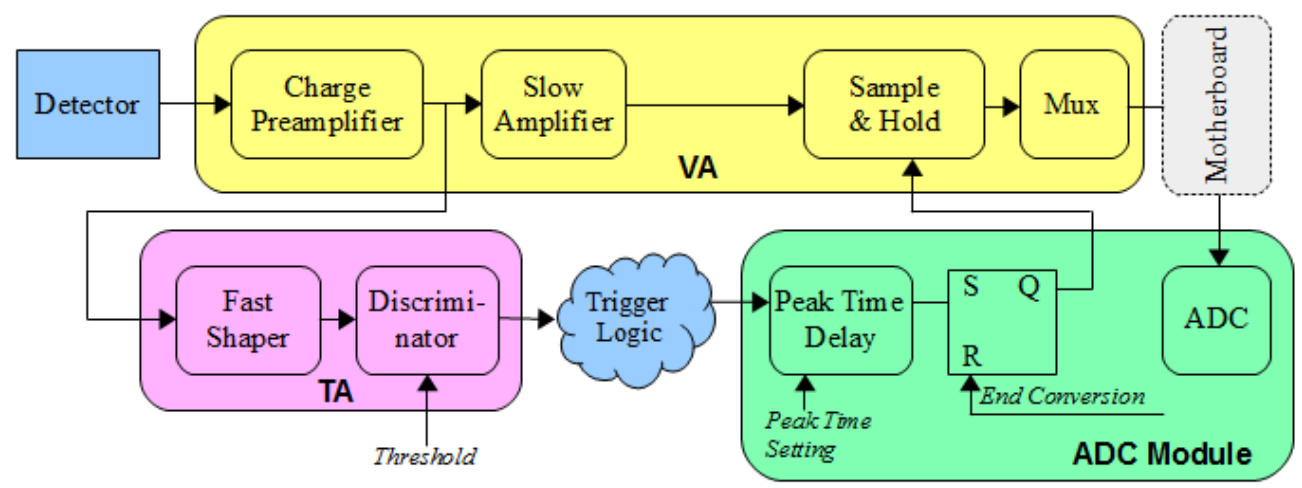

Figure 16. (color online) Schematic block diagram of a single channel of the VA-TA board. The chip VA is essentially used as charge-sensitive preamplifier and slow-shaping amplifier, while the chip TA is used as leading-edge discriminator for the fast-shaped output signal of the VA charge-sensitive preamplifier. A programmable delay unit included in the ADC board, activates a sample-and-hold circuit by a SR-latch (see text for additional details).

This threshold (common to all 32 strips of the same VA-TA board) is externally settable through an Inter-Integrated-Circuit $\left(\mathrm{I}^{2} \mathrm{C}\right)$ module, connected to board with a LEMO cable. A 32-bit mask can be set in order to enable or disable the discriminators of each strip individually. The discriminator output signal (shown in panel (b)) is sent to the TSB that handles the trigger logic and generates the master trigger signal (see Section 7). The master trigger signal then is sent to the "peak-time delay" circuit (see Figure 16), included in a custom ADC (described in Section 6). After a digitally programmable 
Figure 17. (color online) Schematic description of the output data stream generation for a single $E_{\text {res }}$ detector strip. Panel (a) sketches the output signal of the Fast Shaper, while the corresponding output signal of the TA leading-edge discriminator is illustrated in panel (b). At the arrival of a trigger and after a digitally programmable delay, a SR-latch is activated (panel (d)). Panel (c) and (e) represent the output of the VA slow amplifier and the output of the VA sample-and-hold circuit, respectively. Ordinate scales are in arbitrary units. See text for additional details.

delay (usually $2 \mu \mathrm{s}$ ), a Set-Reset (SR)-latch (panel (d)) is enabled and the VA "sample-and-hold" circuit is active until the readout sequence is ended (typically after $\sim 11 \mu \mathrm{s}$ ). Panels (c) and (e) finally display the output signal
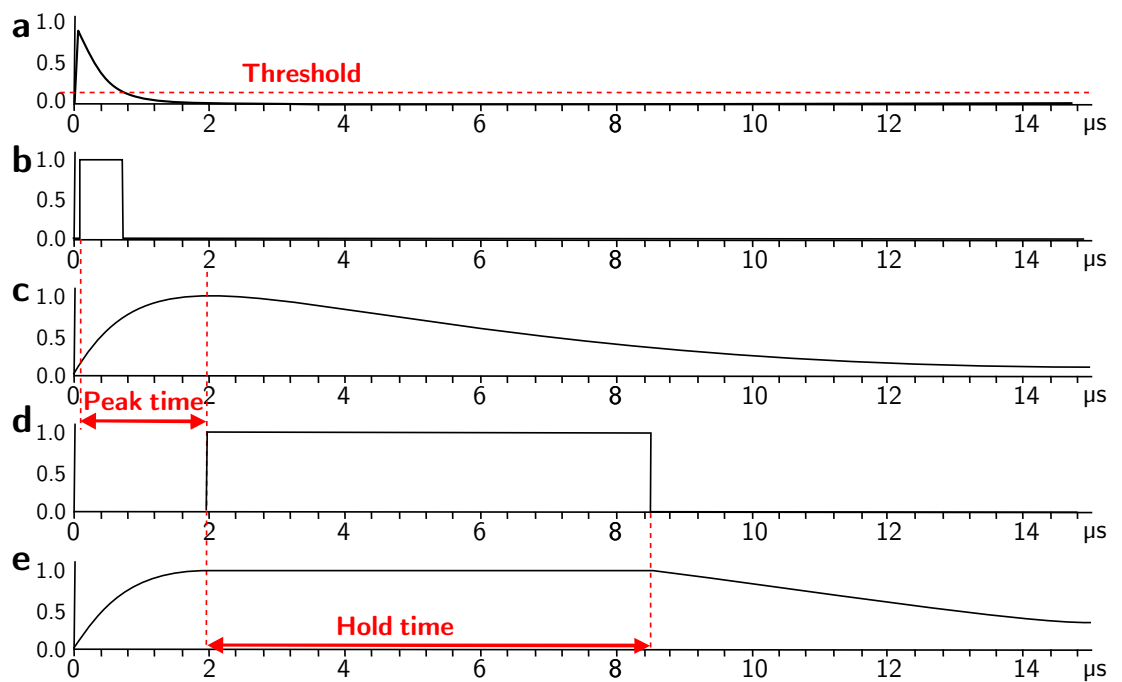

The outputs of the 32 detector strips are then multiplexed in one single signal stream, as in the example shown in Figure 18, and delivered (through the motherboard) to the custom ADC. The ADC samples the input data stream 

strip are gathered.
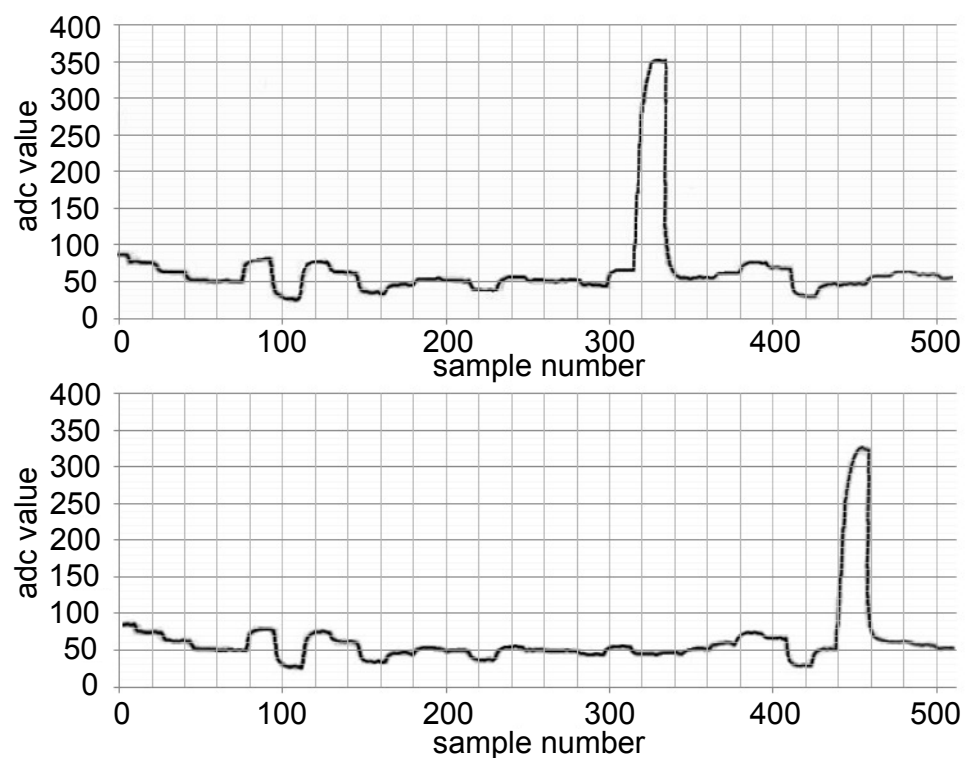

Figure 18. (color online) Examples of two VA multiplexed output signals, corresponding to the detection of two $\sim 45 \mathrm{MeV}{ }^{17} \mathrm{O}$ ions in two different detector strips. Ordinate scales are in arbitrary units.

\subsection{Motherboard}

661

Each detector side requires the use of one VA-TA board, thus the treatment of all electronic signals coming from the eight DSSSDs of the $E_{\text {res }}$ layer needs 16 boards. An additional PCB, called the motherboard (shown in Figure 19), was designed by INFN-Padova to handle simultaneously 8 VA-TA boards. The motherboard contains a Field Programmable Gate Array (FPGA) and superintends several different functions, such as VA and TA chip configuration, temperature monitoring, input/output communication with the chips, the $\mathrm{I}^{2} \mathrm{C}$ 
module, communication with the ADC and the TSB. The motherboard also powers the VA-TA boards and contains a DAC unit for the conversion of the (externally settable) TA thresholds and their subsequent delivery to the boards. Moreover, it has a splitting unit, which accepts a test/pulser input signal, splits it into 8 output signals and sends them individually to the VA-TA boards. The 41 connectors located on a motherboard and their function are presented in Figure 19.

The power consumption of the motherboard and of each VA-TA board are $1.4 \mathrm{~W}$ and $0.8 \mathrm{~W}$, respectively.

\section{$5 \quad$ PPAC readout electronics}

A very important issue for handling the PPAC signals, in order to have a good position resolution and a high detection efficiency, is the noise reduction that can be reached by performing a careful grounding and by using a low-noise fast preamplifier, like the 5-channel fast preamplifier Mod. 3356 [48], placed very close to the PPAC (in vacuum). The major characteristics of this preamplifier are the following: a gain factor $=12$, a noise figure (the ratio of input signalto-noise ratio to output signal-to-noise ratio expressed in decibels $)=1.1 \mathrm{~dB}$, a rise time $=1.2 \mathrm{~ns}$, and input/output impedance $=50 \Omega$.

The cathode signal and the anode signals $x_{1}, x_{2}, y_{1}, y_{2}$, extracted from each end of the delay lines are sent to a Timing Filter Amplifier EG\&G ORTEC Mod. 


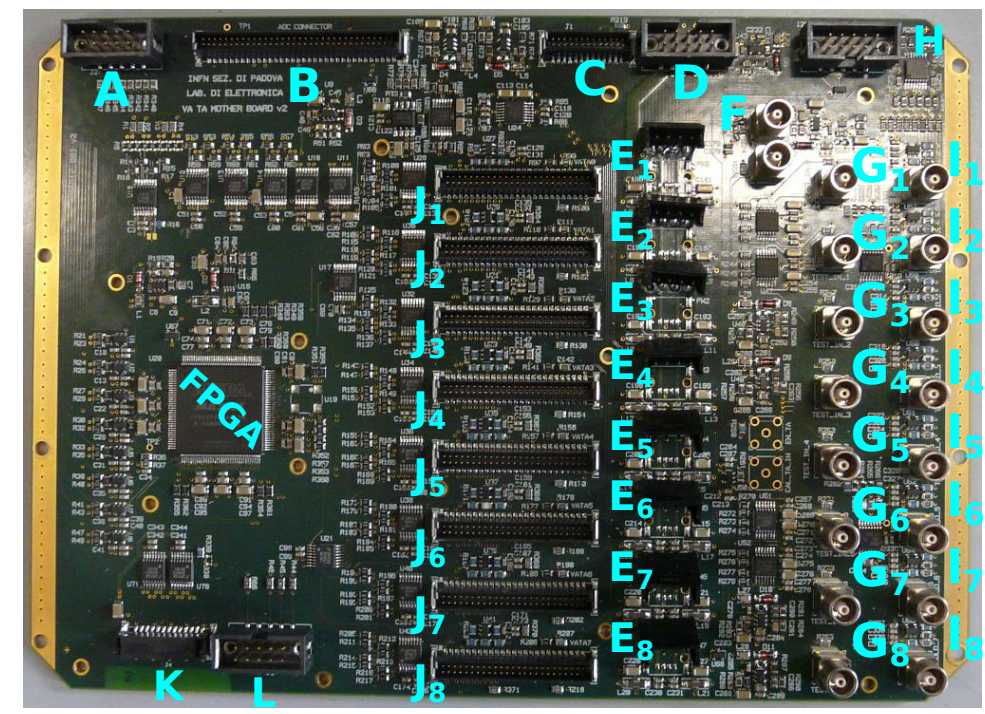

Figure 19. (color online) Image of a Motherboard. Letters label the connectors located on the PCB: A) 10-pin connector for the FPGA configuration; B) 68-pin connector for the input/output digital communication with the ADC; C) 28-pin connector for the analog output signal to the ADC; D) 10-pin connector for the motherboard power supply; $\left.\mathbf{E}_{1}-\mathbf{E}_{8}\right) 8$ 4-pin connectors for powering the VA-TA boards; F) LEMO connector for the test input signal; $\left.\mathbf{G}_{1}-\mathbf{G}_{8}\right) 8$ LEMO connectors for delivering the test signals to the VA-TA boards; H) 10-pin connector for the input/output communication with the $\mathrm{I}^{2} \mathrm{C}$ module; $\left.\mathbf{I}_{1}-\mathbf{I}_{8}\right) 8$ LEMO connectors for delivering the threshold signals to the VA-TA boards; $\left.\mathbf{J}_{1}-\mathbf{J}_{8}\right) 8$ 48-pin connectors for input/output communication with the VA-TA boards; K) 28-pin connector for the communication with the TSB board; L) 10-pin connector for TSB testing purposes. ${ }_{688}$ TFA 474 (cathode) and to fast filter amplifiers EG\&G ORTEC Mod. FTA810 ${ }_{689}$ (anode). The amplified PPAC signals are sent to a MEGAMP constant fraction ${ }_{690}$ discriminator to set a threshold and extract logic signals and, finally, to a ${ }_{691}$ Time to Digital Converter (TDC) Mod. CAEN V775. For handling the high${ }_{692}$ rate fast signals of the PPACs, the $30 \%$ CFD of the MEGAMP module was 
where $k=0.435 \mathrm{~mm} / \mathrm{ns}$ is the slope for the $x$ and $y$ delay lines.

$$
P_{x}=k \frac{t_{x 1}-t_{x 2}}{2}(m m)
$$

$$
P_{y}=k \frac{t_{y 1}-t_{y 2}}{2}(m m)
$$


A custom single-slot VME card (Figure 20) has been developed by INFNMilano [49] to digitize the multiplexed analog signals coming out from the VA-TA boards and from the MEGAMP modules. The ADC card consists of 8 analog differential signal receivers and of 8 12-bit ADC-chip converters, type AD9236, that sample the input signals with a $50 \mathrm{MHz}$ frequency. The use of a high sampling rate enables the determination of when the multiplexed signal is stable enough to be acquired.

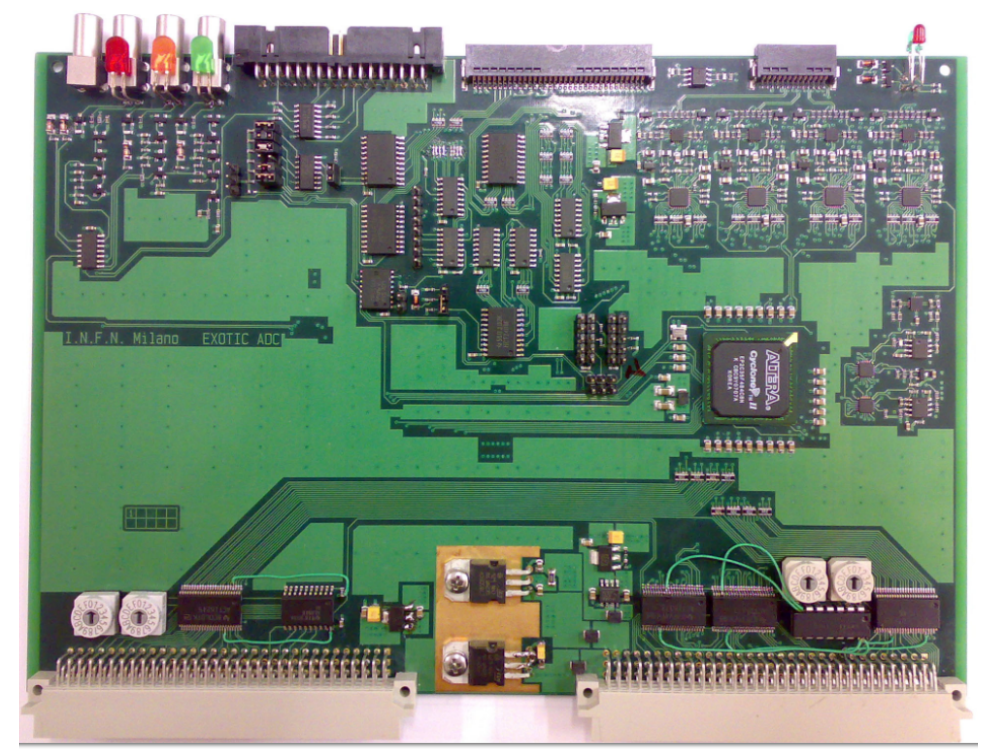

Figure 20. (color online) Picture of the ADC VME module.

The main ADC features are the following:

- 1 unit VME Board;

- 8 differential analog inputs; 
- $\pm 1 \mathrm{~V} \max$ input voltage;

717 - $\pm 1 \mathrm{~V}$ max settable input offset voltage;

- 12-bit resolution;

- Noise $<0.5$ LSB RMS;

720

- Integral non-linearity (for 10\%-90\% of FSR) $< \pm 0.025 \%$;

- Differential non-linearity (for 10\%-90\% of FSR) $< \pm 2 \%$;

- $50 \mathrm{MHz}$ sampling frequency;

- Zero suppression with individual threshold;

\subsection{Functional description}




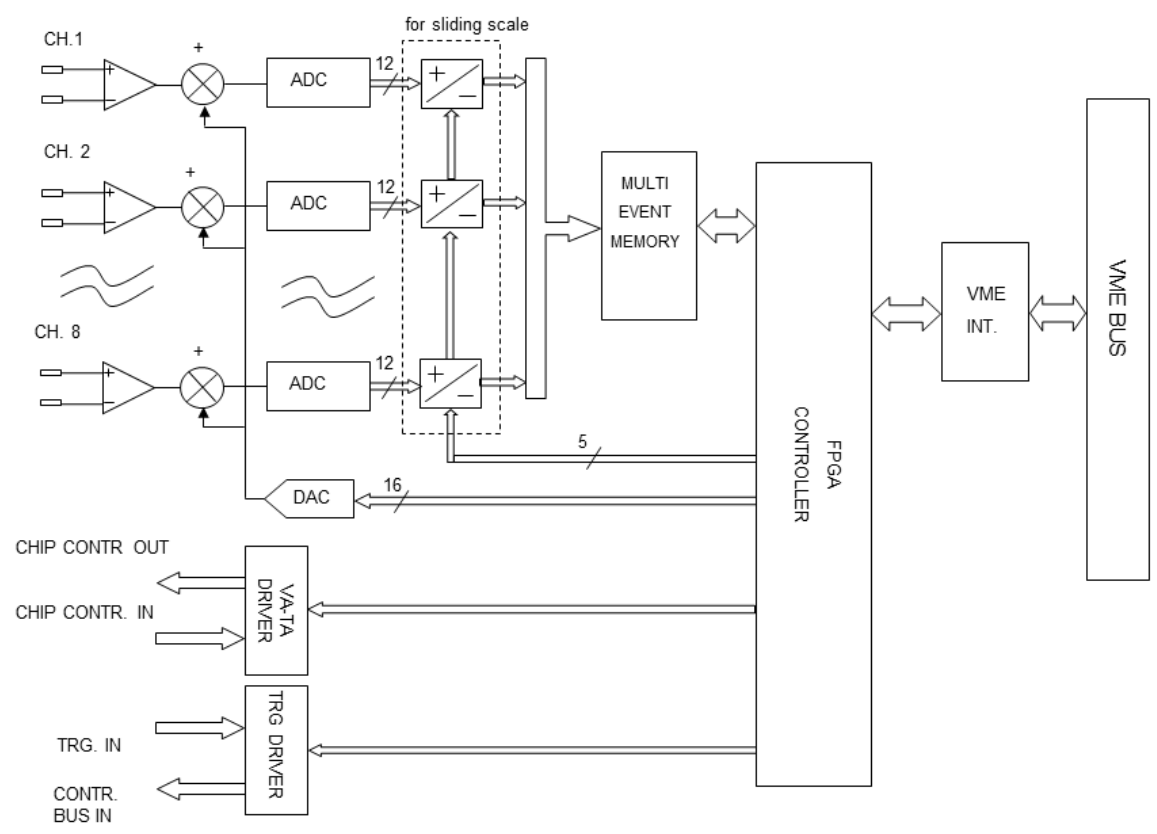

Figure 21. (color online) ADC block diagram.

(see Figure 21 that illustrates the ADC block diagram). The VA Clock (CLK) signal generated from the ADC card controls the multiplexer increment and can reach the maximum frequency of $5 \mathrm{MHz}$ that is programmable so that the analog output is sampled by the ADC with a frequency 10 times higher. In this way the ADC data can be easily processed to reduce noise. During our measurements this modality allows us to find the sample where the signal becomes stable and adjust the sample delay to detect the maximum of the signal. Then, only two or three samples are acquired to reduce the dead time and from the acquired samples only one is used for the data analysis in the end (the other are taken for safety).

To improve the ADC linearity a sliding scale technique circuit was inserted using a 16-bit AD768 DAC. The same DAC is used to set the baseline of 
the ADC-chips. This function is useful because the output signal polarity of the MEGAMP modules is positive while that of the the VA chips can be both positive and negative depending on the processed DSSSD side. For MEGAMP modules the baseline is set near zero channel while for the VA chips the baseline is set at the middle of the range.

The data of all channels are then sequentially stored into an EVENT FIFO memory. Through the VME bus the data can be read in block transfer mode and, at end of the data event, the VME "BERR" (Bus ERRor) signal is asserted.

The board can also handle the test mode of the VA chip. In this modality, an analog pulse with variable amplitude can be addressed to one of the 32 channels of the chip. A monitoring output on the front panel allows an inspection of one of the 8 analog inputs. Moreover, a BUSY output signal is generated by the ADC at the arrival of the external trigger and it is reset at the end of the conversion. The board provides also all the logic signals necessary to the VA-TA chips for the complete event processing and acquisition. Furthemore, it sends a 165-bit initialization stream for the set-up of the TA chip.

An ALTERA CYCLONE II series FPGA handles the card with the 8 ADCchips AD9236, the EVENT FIFO memory, the VME interface and the other logic functions like the BUSY signal generation, the programmable delay and the set-up of the VA-TA chips. 
77

772

The TSB is a general purpose custom VME-standard card, developed by INFN-Napoli. Figure 22 illustrates the TSB PCB. The TSB accepts up to 64 differential TTL input channels for the proposed trigger signals originating from the different detectors and handles the trigger logic of the whole experimental set-up.

The trigger logic is fully programmable via VME. The width and delay of each proposed trigger signal in the input can be modified to compensate for the different TOF of the detected particles, the different time response of the detectors and the different cable delays. Selected input signals can be sent to 16-bit divider units. This function is very useful for normalization purposes when we want to acquire events in "single" modality (multiplicity 1) without increasing the acquisition dead time, for example ions that are elastically scattered from the target and detected with the monitor detectors at forward angles, or PPAC events for monitoring the beam rate and profile. In order to monitor the rate of the different trigger signals, ratemeters are also included in the board. A 16-bit time counter (1 s/bit resolution) is foreseen for measuring the run duration and 16-bit counters for the number of proposed and accepted triggers, allowing us to measure the dead time. For testing purposes, an $\mathrm{ON} / \mathrm{OFF}$ mask of the input channels and a forced-trigger mask can be set by the user. 
811 by an external signal.

Figure 23 illustrates the TSB block diagram. For all the logic functions and for the VME interface, the TSB makes use of 5 FPGAs XILINX series SPARTAN $\left(\mathrm{X}_{a}, \mathrm{X}_{b}, \mathrm{X}_{c}, \mathrm{X}_{4}, \mathrm{X}_{f}\right.$ in Figure 23). Four among the five FPGAs $\left(\mathrm{X}_{a}, \mathrm{X}_{b}, \mathrm{X}_{c}, \mathrm{X}_{4}\right)$ have 16 independent inputs and 4 outputs for monitor signals and diagnostic purposes. The monitor outputs can be connected to 190 internal (total) test points through a multiplexer (denoted Mux in Figure 23) allowing monitoring of the logic generating the trigger along the whole chain. The first three FPGAs $\left(\mathrm{X}_{a}, \mathrm{X}_{b}, \mathrm{X}_{c}\right)$ are related to DSSSD signals, for both the $\Delta E$ and the $E_{\text {res }}$ stage. Each FPGA accepts the OR signal from the strips of the $\mathrm{x}$ (back) side and the OR signal from the strips of the $y$ (front) side, creating an OR/AND logic between the $\mathrm{x}$ and $\mathrm{y}$ sides for eight DSSSDs. The fourth FPGA $\left(\mathrm{X}_{4}\right)$ is related to other detectors (e.g. PPACs, monitor detectors, plastic scintillators, etc).

The trigger philosophy is based on four different levels: the first level handles the DSSSD signals. The second level receives the output signals of the first level and handles the signals of the other detectors (PPACs, monitor detectors, plastic scintillators, etc). The third level receives the second level output signals and can generate further logic. The fourth level $\left(\mathrm{FPGA} \mathrm{X}_{f}\right)$ generates the master trigger signal combining the third level output signals and the detector signals in single modality originating from the dividers (see the divider unit of $\mathrm{X}_{4}$ in Figure 23 ). At this level, the master trigger signal can be vetoed 
signals into TTL ones for the TSB inputs.

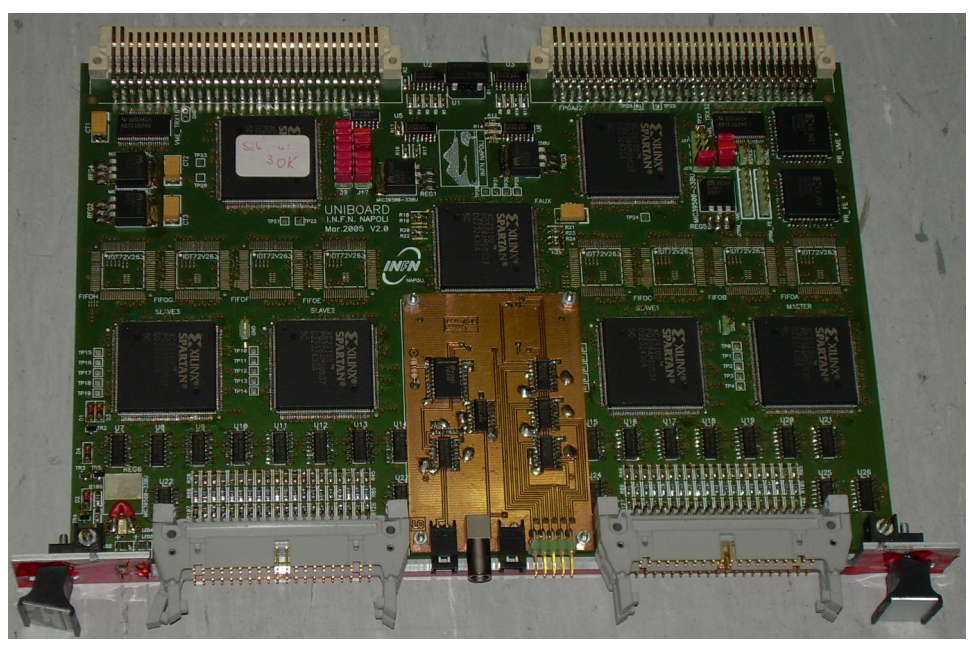

Figure 22. (color online) TSB module

${ }_{814}$ The main features of the TSB are the following:

815

816

817

818

819

820

821

822

823

- 64 differential TTL input signals;

- 16 differential TTL monitor outputs;

- 1 VETO input signal (NIM);

- 1 Master trigger output (NIM);

- Delays of the input signals: $\max 225$ ns step of 12.5 ns;

- Signal width $\max 225$ ns step of 12.5 ns;

- 16-bit counter for the proposed triggers;

- 16-bit counter for the accepted triggers;

- 16-bit time counter for the run duration, step of $1 \mathrm{~s}$. 


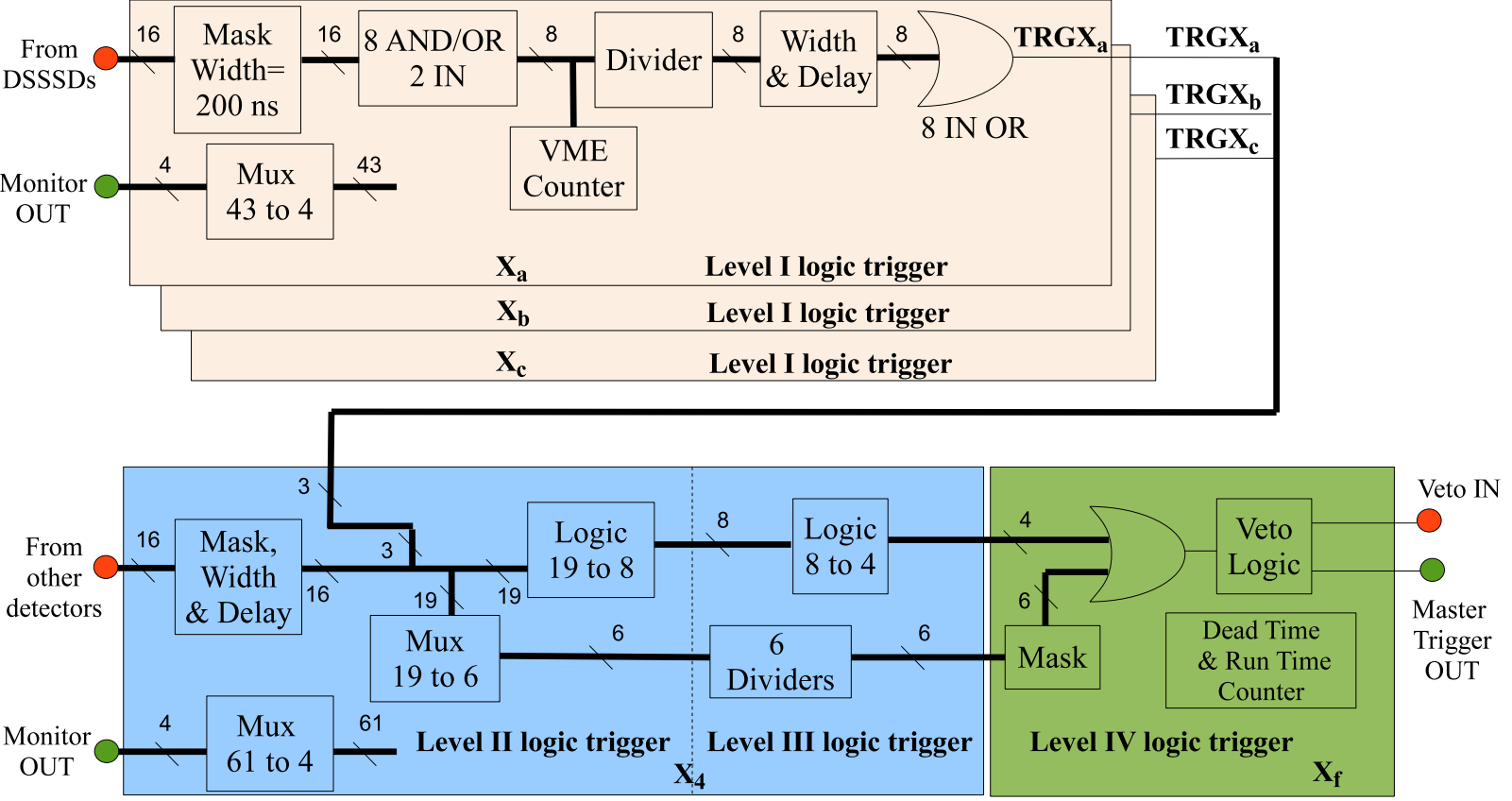

Figure 23. (color online) TSB block diagram. Mux stands for Multiplexer. For details see the text.

The TSB GUI is organized as follows: 


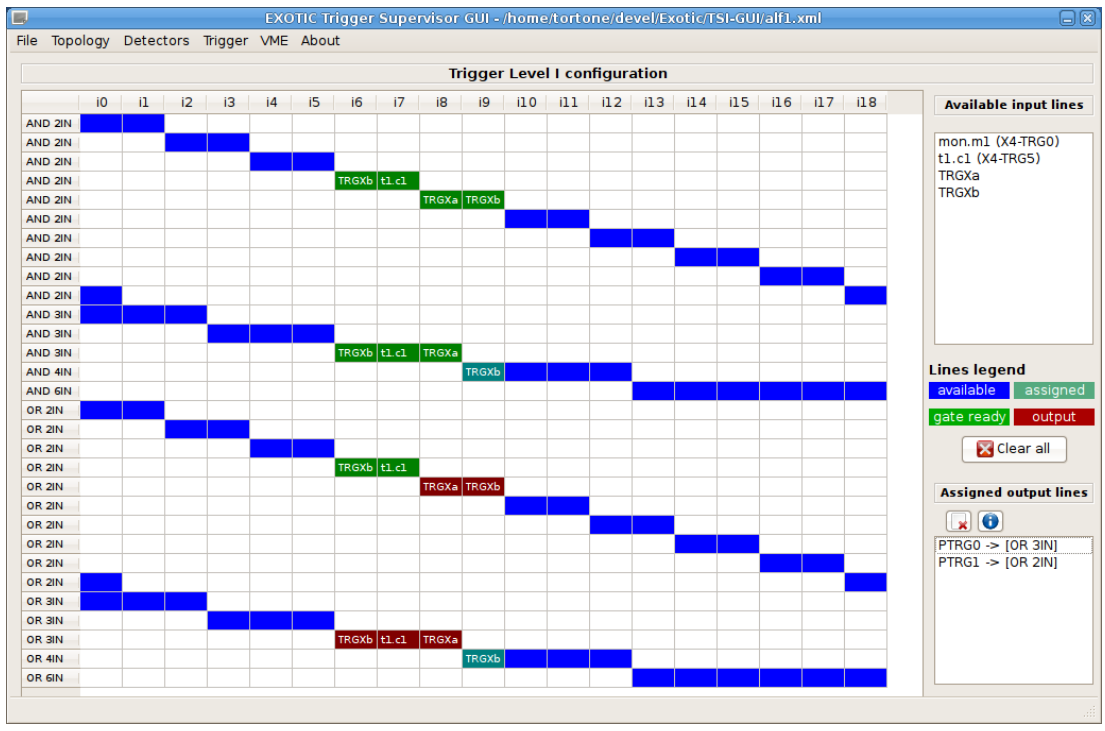

Figure 24. (color online) Trigger Logic definition dialog of the TSB GUI.

Topology: due to the large flexibility of the detection array, the first step required in order to impose a trigger logic is the definition of the topology. In this step the user defines all the detector available in the set-up with a name, a type (DSSSD, PPAC, monitor detectors, etc) and the related telescope.

Wiring and Set-up: this step is required to connect each detector output line with a TSB input line. It is also possible to specify for each DSSSD an AND/OR logic between the signals of the $\mathrm{x}$ and $\mathrm{y}$ sides and a set of parameters like signal width/delay.

Trigger Logic: After topology, wiring and set-up definition, the user can define the trigger logic to apply on the TSB input set. The definition of the trigger logic concerns different levels as explained in the previous paragraph. To simplify this task a "drag and drop" dialog was created. Figure 24 illustrates the window dialog for the definition of the trigger logic. The user can 


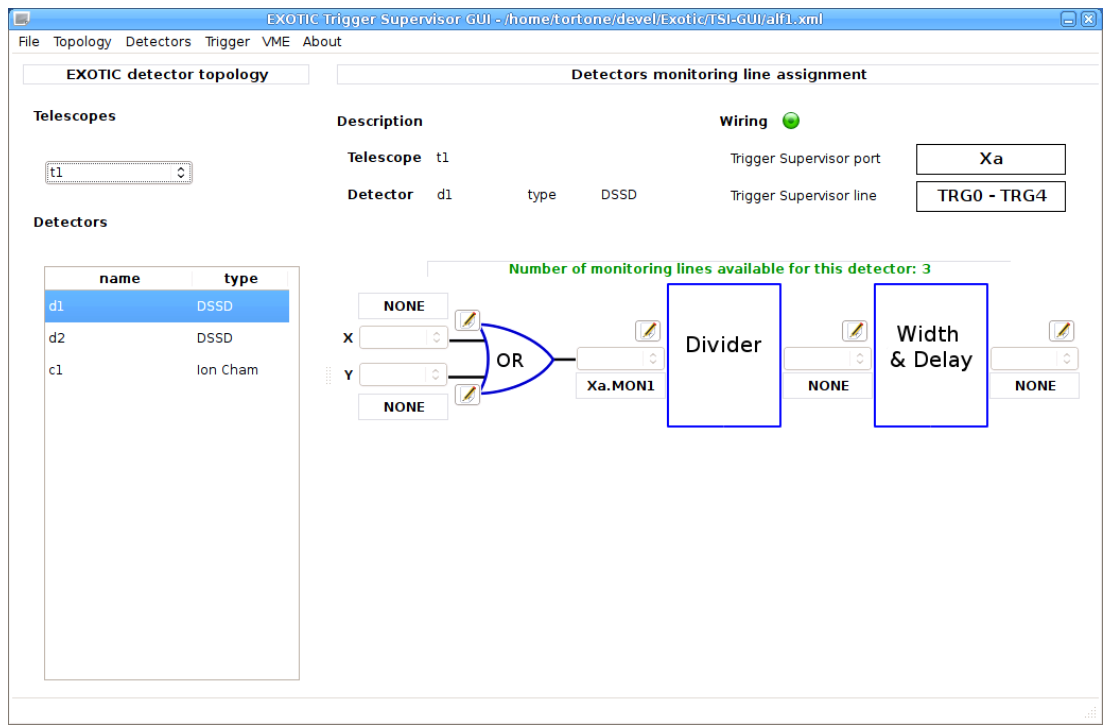

Figure 25. (color online) Monitoring lines can be assigned before or after each detector signal processing step to inspect shape and timing (width, delay).

849 A dedicated dialog was also implemented for the definition of the TSB monitoring lines (see Figure 25). The user can exploit a monitoring line to inspect ${ }_{851}$ a signal within the TSB, analyzing it outside with an oscilloscope or other ${ }_{852}$ equipment. The monitoring line definition is available for signals related to a 853 detector or for various internal logic signals.

${ }_{854}$ The TSB GUI is also equipped with a control panel useful to test and monitor

${ }_{857}$ it before the run. During a run, the control panel is useful to monitor the 


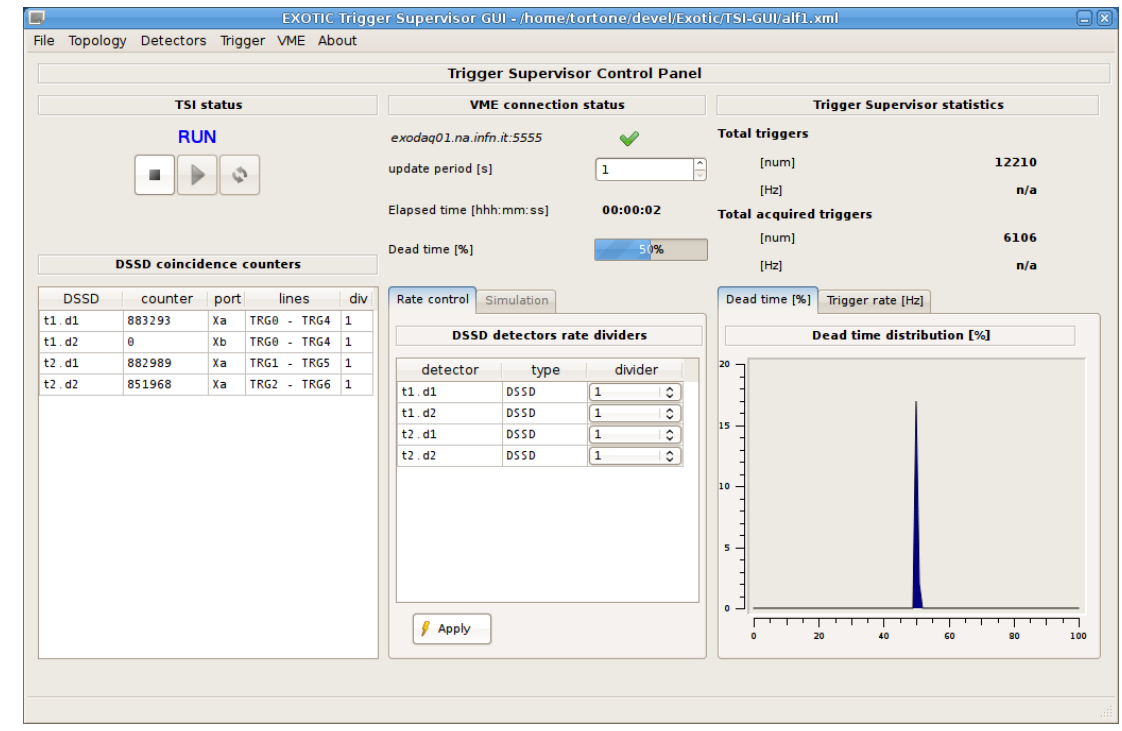

Figure 26. (color online) The TSB GUI control panel is useful to test and monitor the trigger logic. A dialog window displays a test run status.

In Figure 26 a dialog window displays a test run status. Data are polled at a user defined frequency through network from the Vme2Net service, running on the Data Acquisition workstation.

\section{Data Acquisition System (DAQ)}

The custom ADCs and the TSB are housed in a VME crate connected to the acquisition computer through a commercial CAEN VME-bridge Mod. V2718.

Data monitoring and acquisition software consists of a series of applications schematically shown in Figure 27. The XDAQ [50] application handles the 

872 alization.

873

874

communication between the acquisition computer and the VME boards, such as custom ADCs and TSB, CAEN Mod. V775 TDCs and CAEN Mod. V785 ADCs. The XDAQ also executes the readout procedures, by storing data on disk and simultaneously sending them through a TCP socket for on-line visu-

The Run Control and Monitoring System (RCMS [51]) is a net platform that can be remotely handled by the user through an internet connection, since the RCMS GUI is web-browser based. The RCMS application is used for configuring the different VME modules and the programmable registers of the motherboards, for the acquisition run control and for displaying relevant online information (acquisition status, event rate, output data file size, ...). Both RCMS and XDAQ were developed at CERN in collaboration with INFN-LNL and are currently adopted in the CMS experiment $[50 ; 51]$.

On-line spectra are visualized by means of the computer program CRACOW [52]. This software essentially consists of:

- a "spy" program, connected to the XDAQ system through the TCP socket, to store to disk a user-defined list of 1D- and 2D-spectra. Alternatively, CRACOW can also be employed for the off-line analysis, by directly accessing a previously saved data file;

- a GUI program, to display user-defined spectra, to control some spy options, such as deleting spectra, creating new ones, defining 1D- or 2D-gates and, 
finally, to provide some basic analysis tools such as peak integration and calibration.

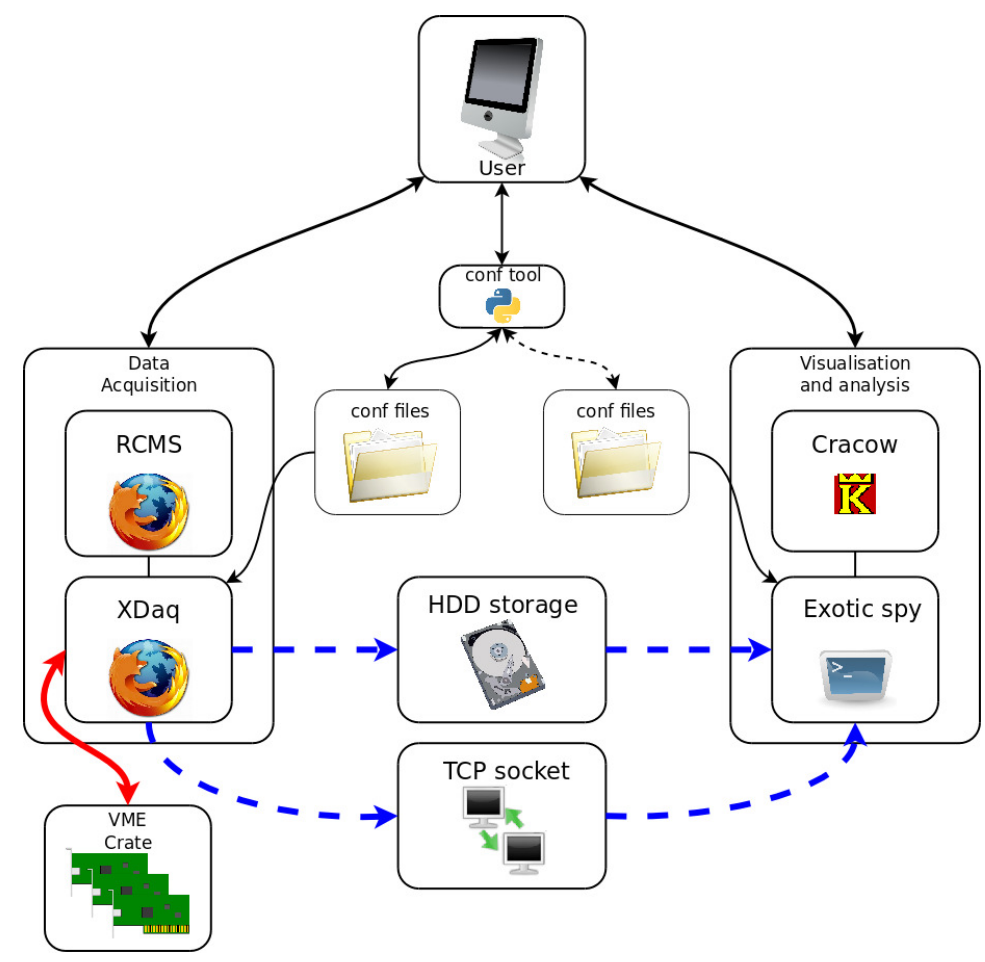

Figure 27. (color online) Schematic view of the acquisition software used for the detection array and interconnections between the computer programs involved.

\section{Offline tests}

The performance of the PPACs, ICs, DSSSDs and the newly developed electronics were at first tested offline employing standard $\alpha$ sources $\left({ }^{239} \mathrm{Pu}^{2}{ }^{241} \mathrm{Am}-\right.$ ${ }^{244} \mathrm{Cm}$ ) with the following energies (intensities): $\mathrm{E}_{\alpha}=5.157 \mathrm{MeV}(73.3 \%), 5.144$ $\mathrm{MeV}(15.1 \%)$ and $5.106 \mathrm{MeV}(11.5 \%)$ for the ${ }^{239} \mathrm{Pu}, \mathrm{E}_{\alpha}=5.486 \mathrm{MeV}(84.5 \%)$, $5.443 \mathrm{MeV}(13.0 \%)$ and $5.388 \mathrm{MeV}$ (1.6\%) for the ${ }^{241} \mathrm{Am}$ and $\mathrm{E}_{\alpha}=5.805 \mathrm{MeV}$ (76.4\%) and 5.763 $\mathrm{MeV}(23.6 \%)$ for the ${ }^{244} \mathrm{Cm}$. 

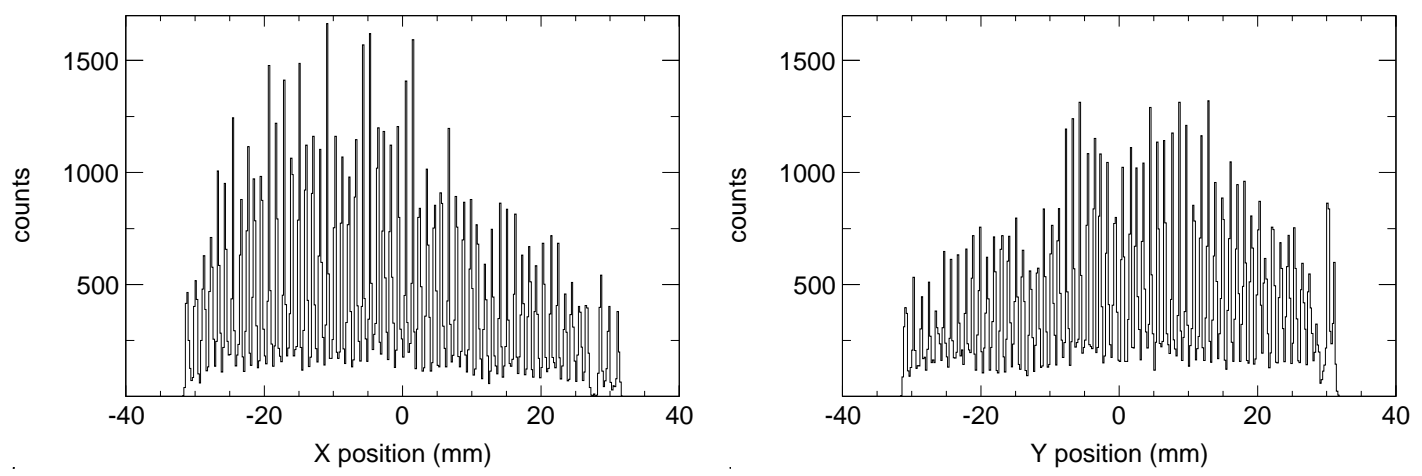

Figure 28. (color online) $x$ and $y$ position obtained with a PPAC for an ${ }^{241} \mathrm{Am} \alpha$ source. The wire spacing is $1 \mathrm{~mm}$ in both $x$ and $y$ direction.

The PPAC was illuminated with an ${ }^{241} \mathrm{Am} \alpha$ source situated at a distance of about $30 \mathrm{~cm}$. The gas used was $\mathrm{C}_{4} \mathrm{H}_{10}$ and the operating pressure 20 mbar.

The IC performance was tested with an ${ }^{241} \mathrm{Am}$ source, positioned at 22.5 $\mathrm{cm}$ from the detector and collimated with a $3 \mathrm{~mm}$-diameter hole. The IC was filled with $\mathrm{CF}_{4}$ gas at 61.5 mbar. The cathode (anode) bias was $-300(+100) \mathrm{V}$ and the measurement was done with $1 \mu$ s shaping time. According to energy loss calculations performed with LISE [53] (parameterization based on [54]), $\alpha$ particles with an average energy of $5.479 \mathrm{MeV}$ are expected to deposit in 


$$
\delta(\Delta E)_{\text {stat }}=2.35 \sqrt{F w \Delta E}=8 \mathrm{keV}
$$
window has been taken into account). expected to contribute to $\delta(\Delta E)_{\text {exp }}$ : $\mathrm{CF}_{4}$ gas: average $0.996 \mathrm{MeV}$ in the IC active volume (the energy loss in the mylar

Figure 29 shows the energy loss $\Delta E$ of the $\alpha$ particles in the gas fitted by a Gaussian curve. The FWHM is $\delta(\Delta E)_{\text {exp }}=73 \mathrm{keV}$ resulting in a FWHM overall resolution $R_{\text {exp }}=\delta(\Delta E)_{\exp } / \Delta E=7.3 \%$. Different terms (FWHM) are

- the statistical fluctuation of the number of created charge carriers in the

with $F=0.2$ the Fano factor and $w=54 \mathrm{eV}$ [55] the mean energy for the creation of an electron-ion pair in the gas.

- electronic noise: the FWHM expected preamplifier noise coupled with the IC (which has a capacitance of $19 \mathrm{pF}$ ) is $\delta(\Delta E)_{\text {th,noise }}<26 \mathrm{keV}$ at $3 \mu \mathrm{s}$ shaping time. In Section 3.1 the expected preamplifier noise is given for a silicon detector, thus, the noise for the IC was obtained by multiplying this value with the ratio of $w$ for the $\mathrm{CF}_{4}$ gas to that for a silicon detector, i.e. $54 \mathrm{eV} / 3.6 \mathrm{eV}$. The FWHM measured noise of the electronic chain, obtained with the signal of a pulser, was found to be $\delta(\Delta E)_{\text {exp }, \text { noise }}=33 \mathrm{keV}$ at $1 \mu \mathrm{s}$ shaping time.

- $\delta(\Delta E)_{s t r}=55 \mathrm{keV}(\mathrm{FWHM})$ due to the energy straggling of the $\alpha$ particle 


$$
\delta(\Delta E)_{s t r}=2.35 k Z_{P} \sqrt{Z_{P} t / A_{T}}(M e V)
$$

933

$$
\delta(\Delta E)_{t o t, t h}=\sqrt{\delta(\Delta E)_{\text {stat }}^{2}+\delta(\Delta E)_{\text {str }}^{2}+\delta(\Delta E)_{\text {exp }, \text { noise }}^{2}}
$$

943

where $Z_{P}$ is the atomic number of the projectile, $Z_{T}$ and $A_{T}$ the atomic and mass numbers of the material, respectively, and the thickness in $\mathrm{g} / \mathrm{cm}^{2}$ : The parameter $k$ increases logarithmically with incident energy, and is parameterized from the experimental data. Its value ranges approximately from 1 (at $1 \mathrm{MeV} /$ nucleon) to 2.5 (at $1 \mathrm{GeV} /$ nucleon);

- The $\Delta E$ variation due to energy straggling of the $\alpha$ particles in the mylar window was found to be negligible.

By taking into account all the above contributions, the FWHM overall expected resolution $\delta(\Delta E)_{t o t, t h}$ can be found with the following formula:

that gives $\delta(\Delta E)_{t o t, t h}=65 \mathrm{keV}$ and a FWHM resolution of $R_{t o t, t h}=6.5 \%$, very close to the experimental value $R_{\text {exp }}=7.3 \%$.

By subtracting the measured electronic noise from the overall experimental 7 resolution we derive the IC FWHM intrinsic resolution: 


$$
\Delta E_{\text {intr }}=\sqrt{\delta(\Delta E)_{\text {exp }}^{2}-\delta(\Delta E)_{\text {exp }, \text { noise }}^{2}}=65 \mathrm{keV}
$$

The overall resolution obtained here, $R_{\text {exp }}=7.3 \% \mathrm{keV}$ is comparable with the 950 $\sim 7 \%$ value of an axial device [40] for similar energy loss of $\alpha$ particles origi951 nating from a ${ }^{241} \mathrm{Am}$ source in a $\mathrm{P} 10\left(90 \% \mathrm{Ar}+10 \% \mathrm{CH}_{4}\right)$ gas.

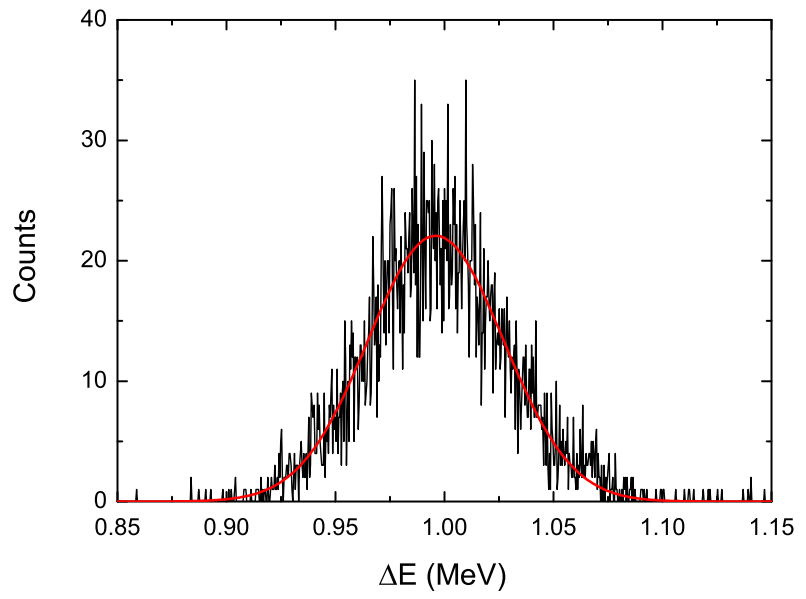

Figure 29. (color online) Energy loss $\Delta E$ of $\alpha$ particles emitted from an ${ }^{241} \mathrm{Am}$ source in the IC. The red line is the result of a Gaussian fit of the experimental data. A FWHM overall resolution of $73 \mathrm{keV}(7.3 \%)$ was achieved for $\Delta E=0.996$ $\mathrm{MeV}$ and a FWHM intrinsic resolution of $65 \mathrm{keV}$ (6.5\%) (for details see the text).

${ }_{953}$ The offline tests of a $\Delta E$ stage $43 \mu$ m-thick DSSSD module were done by 954 placing an ${ }^{241} \mathrm{Am}-{ }^{244} \mathrm{Cm}$ composite $\alpha$ source at about $15 \mathrm{~cm}$ from the detec- 
tor. The DSSSDs were overbiased at $-10 \mathrm{~V}$ (nominal depletion voltage: $-6 \mathrm{~V}$ ) and maximum leakage currents up to $20 \mathrm{nA}$ were measured. Front (junction) and back (ohmic) sides of the detectors were alternately irradiated and no significant differences were observed in the energy spectra.

Figure 30 shows a typical spectrum collected with two short-circuited strips during the tests. A FWHM overall energy resolution $\delta E_{\text {exp }}=38 \mathrm{keV}(0.65 \%)$ was achieved for the detection of $5.805 \mathrm{MeV} \alpha$ particles illuminating the front side of the DSSSD (at $3 \mu$ s shaping time).

The FWHM expected electronic noise of the preamplifier for two short-circuited strips (capacitance of $680 \mathrm{pF}$ ) is $\delta E_{\text {th,noise }}<15.5 \mathrm{keV}$ (at $3 \mu$ s shaping time) while the FWHM measured noise of the electronic chain, obtained with a pulser signal, was found to be $\delta E_{\text {exp,noise }}=16 \mathrm{keV}$ (at $3 \mu$ s shaping time). By subtracting in quadrature the measured electronic noise from $\delta E_{\text {exp }}$ we obtain a FWHM intrinsic resolution equal to $34 \mathrm{keV}$ (0.59\%), compatible with the FWHM intrinsic resolution taken from the datasheet of the MICRON Semiconductor Ltd. (about 36-40 keV for two strips of the considered detector and for $\alpha$ particles of an ${ }^{241} \mathrm{Am}$ source). We remark here that the overall resolution of the chain (detector+electronics) is dominated by the detector intrinsic resolution.

For measuring the time resolution of the whole chain (detector+preamplifier+MEGAMP CFD), an ${ }^{241} \mathrm{Am} \alpha$ source was employed. A preamplifier signal with 28 ns rise 


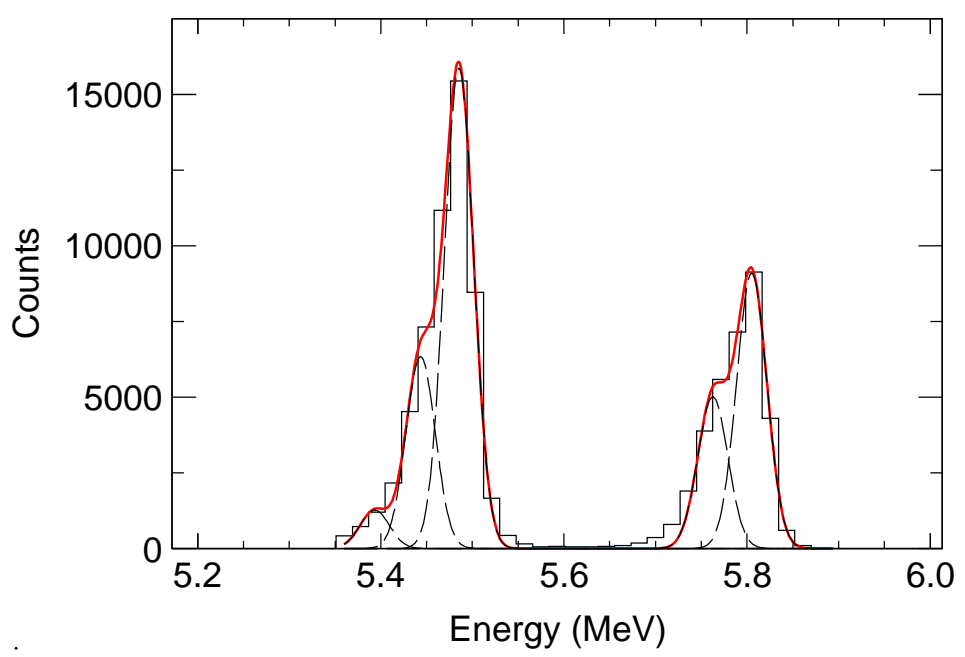

Figure 30. (color online) Energy spectrum of $\alpha$ particles emitted from an ${ }^{241} \mathrm{Am}$ - ${ }^{244} \mathrm{Cm}$ composite source and detected with two short-circuited strips of the $\Delta E$ DSSSD. The $\alpha$ particles impinge on the front side of the detector. The red line is a result of a 5-Gaussian fit of the experimental data. For $\alpha$ particles with $\mathrm{E}_{\alpha}=5.805 \mathrm{MeV}$ a FWHM overall energy resolution of $38 \mathrm{keV}(0.65 \%)$ was achieved. By subtracting the FWHM measured electronic noise, we deduce a FWHM intrinsic resolution of $34 \mathrm{keV}(0.59 \%)$. time was sent to the MEGAMP module, set in this modality: START with 


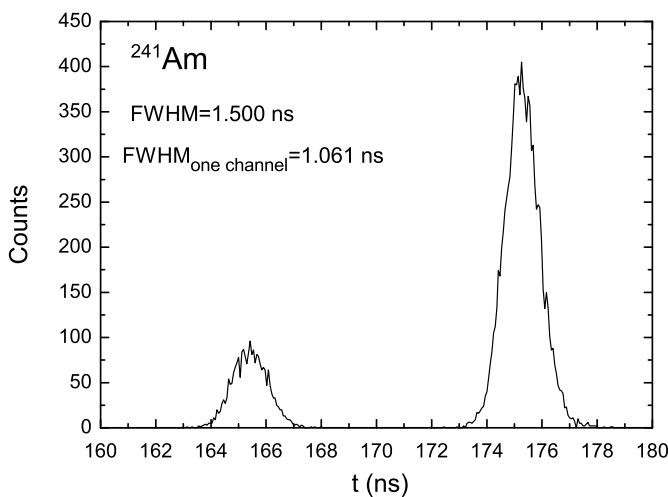

CFD to the 1.061 ns FWHM overall time resolution of the chain, the previous measurement was repeated using an input signal from a Reference Pulse Module Canberra with $20 \mathrm{~ns}$ rise time and $500 \mathrm{mV}$ amplitude. A FWHM equal to 0.100 ns was obtained (right-hand side of Figure 31), corresponding to a FWHM jitter time of 0.071 ns for a single CFD channel, in very good agreement with the module specifications previously presented.

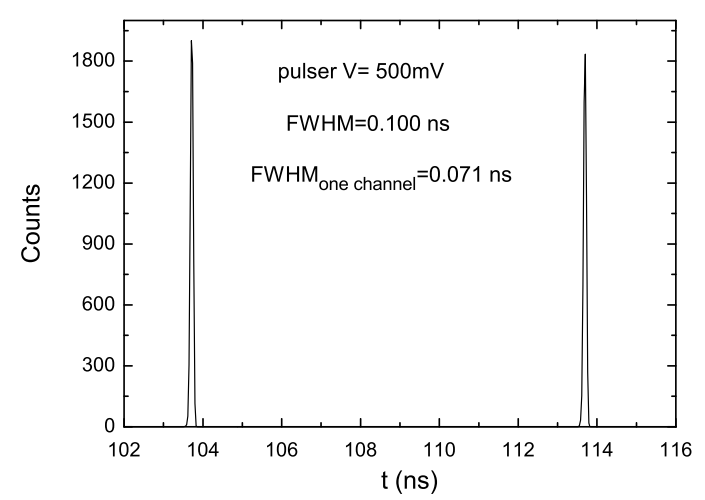

Figure 31. (color online) Left-hand side: TAC spectrum of the chain (43 $\mu$ m-thick DSSSD + preamplifier+MEGAMP CFD) obtained by using an ${ }^{241} \mathrm{Am} \alpha$ source: START signal provided by the $30 \%$ CFD output, STOP signal from the (delayed) 80\% CFD output of the MEGAMP. Right-hand side: Same as in the left panel, but for a pulser signal with $20 \mathrm{~ns}$ rise time and $500 \mathrm{mV}$ amplitude. Relative calibration of the TAC spectra was done by adding a known delay of 10 ns. For additional details see text. For the offline tests of the $E_{\text {res }}$ modules, a ${ }^{239} \mathrm{Pu}-{ }^{241} \mathrm{Am}-{ }^{244} \mathrm{Cm}$ composite $\alpha$ source was placed at about $4-5 \mathrm{~cm}$ from the detector surface. Both the front 
and the back side of the detector were exposed to $\alpha$ particles without observing remarkable differences in the measured spectra, hence all the tests described in the following were performed with $\alpha$ particles impinging on the front side. The detectors were biased at $+50 \mathrm{~V}$ (nominal depletion voltage: $+24 \mathrm{~V}$ ) from the back side, whereas the front side was kept at ground.

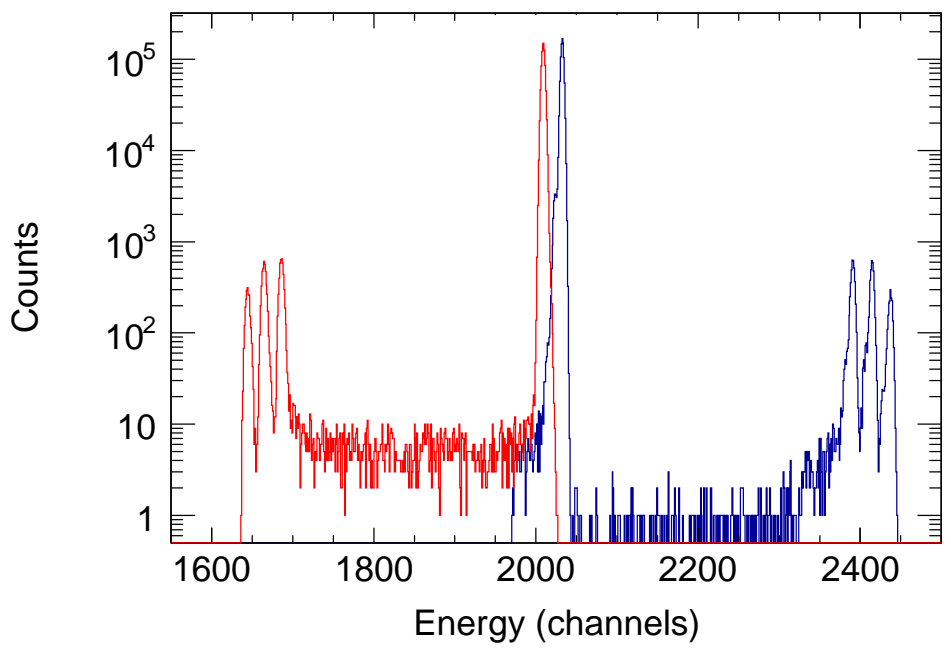

Figure 32. (color online) Tests of the $E_{\text {res }}$ DSSSD with a ${ }^{239} \mathrm{Pu}^{2}{ }^{241} \mathrm{Am}-{ }^{244} \mathrm{Cm}$ composite $\alpha$ source. The $\alpha$ particles impinge on the front side of the detector. Superimposition of the negative $\alpha$ energy spectrum (in red) measured from a single strip on the back side and the positive $\alpha$ energy spectrum (in blue) measured from a strip on the front side of an $E_{\text {res }}$ DSSSD. The ASIC electronics and the ADCs in use made possible the simultaneous measurement of signals with opposite polarities. See text for additional details.

Figure 32 shows the superimposition of the negative $\alpha$ energy spectrum (in 1000 red) measured from a single strip of the detector back side and the positive $\alpha$ 1001 energy spectrum (in blue) collected by a single strip of the detector front side. 

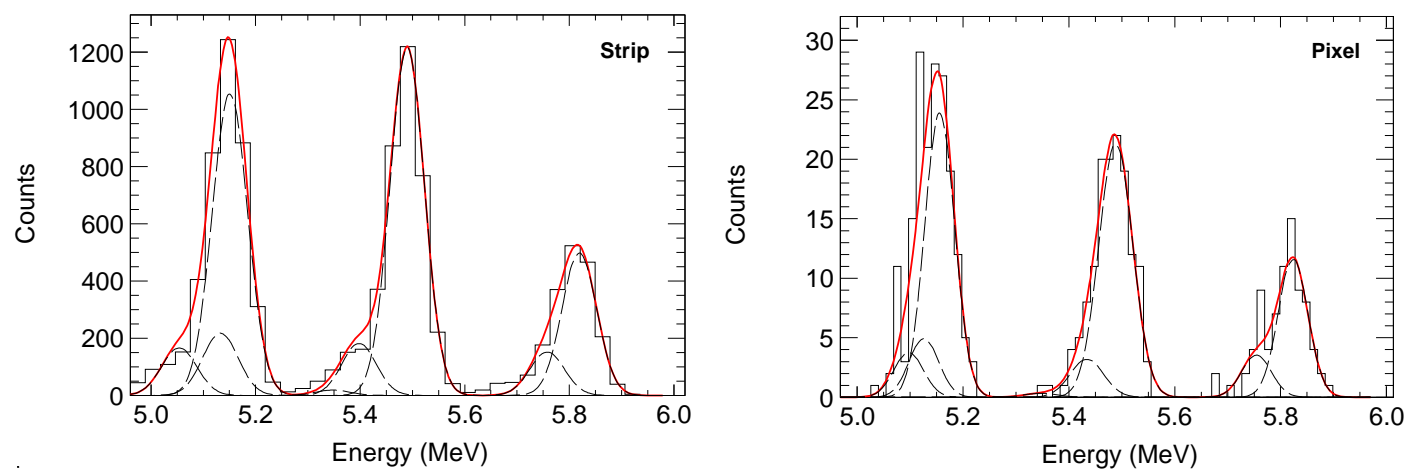

Figure 33. (color online) (a) Enlargement of the positive portion of the spectrum shown in Figure 32, energy calibrated. The red line is a result of a 8-Gaussian fit of the experimental data; (b) As in (a) for a pixel of the detector. For $\alpha$ particles with $\mathrm{E}_{\alpha}=5.805 \mathrm{MeV}$ a FWHM overall energy resolution of $66 \mathrm{keV}(1.14 \%)$ was achieved which, by subtracting the measured electronic noise, corresponds to a FWHM intrinsic resolution of $33 \mathrm{keV}(0.57 \%)$.

energy, was set for this measurement. This figure gives an example of the ADC capabilities (see Section 6) to simultaneously record signals of opposite polarities. Figure 33a displays an enlarged portion of the positive energy spectrum of Figure 32. For $\alpha$ particles with $\mathrm{E}_{\alpha}=5.805 \mathrm{MeV}$ a FWHM overall energy resolution $\delta(\Delta E)_{\exp }=80 \mathrm{keV}(1.38 \%)$ was achieved for the front side and 105 $\mathrm{keV}(1.81 \%)$ for the back side. The better energy resolution for the detector front side is related to the fact that this side (kept at ground potential) is less sensitive to instabilities and fluctuations generated by the power supply. A contribution to the obtained energy resolution for the detector strip, originates from the energy broadening of the $\alpha$ particles crossing the detector dead layer (1 $\mu \mathrm{m}$ silicon equivalent layer) with very different angles (due to the small distance between the source and the DSSSD). To avoid this broadening, 
we consider in Figure 33b the same spectrum as in Figure 33a for a pixel of the front side. The achieved FWHM overall resolution becomes $\delta E_{\text {exp }}=66 \mathrm{keV}$ (1.14\%) for the front side and $85 \mathrm{keV}(1.46 \%)$ for the back side.

The FWHM expected noise of the VA chip for 40-45 pF (the capacitance of 1 strip) is about $14 \mathrm{keV}$. However, this theoretical value was never observed neither during tests in the laboratory nor when the detectors were installed at the scattering chamber of the EXOTIC facility. For the measurement shown in Figure 33, the FWHM measured electronic noise (obtained with a pulser signal sent to the VA chip) was $\delta E_{\text {exp,noise }}=57 \mathrm{keV}$. By subtracting in quadrature $\delta E_{\text {exp,noise }}$ from the $\delta E_{\text {exp }}$ of the DSSSD front side (Figure 33b) we deduce a FWHM intrinsic resolution $\delta E_{\text {intr }}=33 \mathrm{keV}(0.57 \%)$, compatible with the typical values of $25 \mathrm{keV}$ given by the datasheets of the Micron Semiconductor Ltd. for a strip of the $300 \mu \mathrm{m}$-thick detectors.

Figure 34 shows the energy correlation plot of two adjacent strips of the detector back side (left panel) and two adjacent strips of the detector front side (right panel) for $\alpha$ particles coming from a ${ }^{239} \mathrm{Pu}-{ }^{241} \mathrm{Am}-{ }^{244} \mathrm{Cm}$ composite source. The events that lie inside the red circles in the figure are the "full energy events", corresponding to particles entering the detector through the central region of a strip and releasing the entire kinetic energy only in this strip. The small number of events (2-3\% of the total) in which a signal is produced in both adjacent strips, are the interstrip events that correspond to particles entering the detector through the region of separation between two 


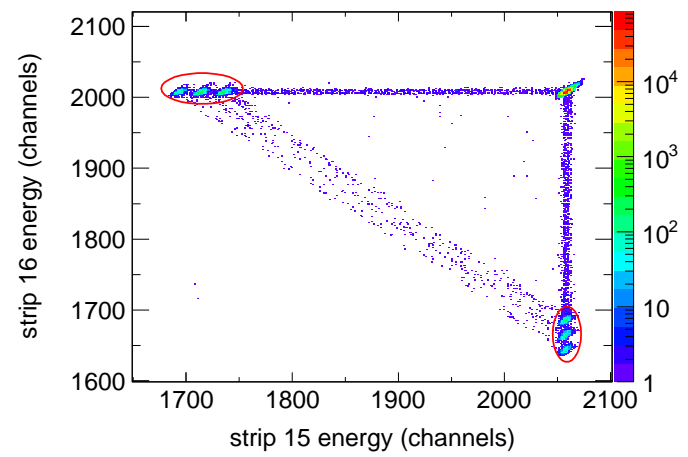

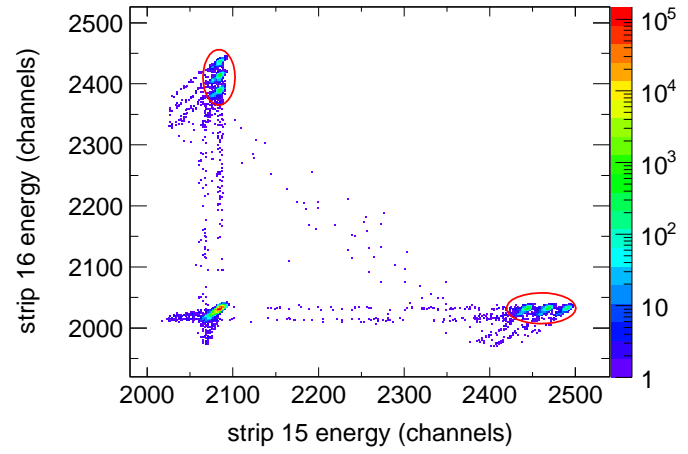

Figure 34. (color online) Correlation plot for the energy deposition of $\alpha$ particles coming from a ${ }^{239} \mathrm{Pu}-{ }^{241} \mathrm{Am}-{ }^{244} \mathrm{Cm}$ composite source in two adjacent strips of the back (left panel) and front (right panel) side of an $E_{\text {res }}$ DSSSD. The $\alpha$ particles impinge on the front side of the detector. The events that lay inside the red circles are the "full energy events". For details see text. 


\section{In-beam performances}

\subsection{PPAC}

The PPAC performance with light ions was obtained in the first experiments with the RIBs delivered by the EXOTIC facility, ranging from ${ }^{8} \mathrm{Li}$ to ${ }^{17} \mathrm{~F}$. In connection with the low RIB intensity, a key feature of the PPAC is the tracking efficiency $\eta_{x y}$, defined as the ratio between the number of particles detected by both the anodes and the number of particles detected by the cathode, that should be as high as possible. This is a quite challenging requirement for the small energy losses of the light ions involved in the considered cases.

The tracking efficiency depends essentially on the signal-to-noise ratio. In test conditions with $\alpha$ sources and with a very low environmental noise is $\eta_{x y} \sim 98 \%$.

However, this is not always the case in real experimental conditions. To obtain $\eta_{x y}$ in the running conditions at the EXOTIC facility, we placed a silicon detector downstream the PPAC at the reaction target position and we triggered the DAQ with the silicon energy signal. $\eta_{x y}$ is given by the ratio: $\eta_{x y}=N_{x y} / N_{\text {silicon }}$, with $N_{\text {silicon }}$ the events acquired in the silicon detector energy spectrum and $N_{x y}$ the events acquired in the PPAC TOF spectrum (START signal provided by the PPAC cathode signal, STOP signal coming from the silicon detector) and simultaneously in the 2D $x-y$ correlation plot. In these conditions, an efficiency $\eta_{x y}=98.5 \%$ was measured for the ${ }^{15} \mathrm{O}$ RIB particles produced at 
$E_{l a b}=31 \mathrm{MeV}$ with a rate of $10^{4} \mathrm{~Hz}$ and $\eta_{x y}=94 \%$ was obtained for a ${ }^{8} \mathrm{~B}$ RIB produced at $E_{l a b}=35 \mathrm{MeV}$ with a rate of $10^{3} \mathrm{~Hz}$ [59]. We should note that $\eta_{x y}$ decreases monotonically as the counting rate increases. This is due to the delay-line employed for the position determination. For a $10^{5} \mathrm{~Hz}$ rate of the produced RIB, the dead time caused by a 138 ns total delay line is $1.4 \%$ while it increases up to $14 \%$ for the maximum expected rate of $10^{6} \mathrm{~Hz}$. However, the use of a multi-hit TDC avoids this loss of efficiency.

Figure 35 shows the profile of a ${ }^{15} \mathrm{O}$ RIB at $E_{l a b}=31 \mathrm{MeV}$ obtained with the PPAC B. The trigger signal was the OR between the signal of a $300 \mu$ m-thick DSSSD placed at $0^{\circ}$ at the end of the EXOTIC reaction chamber and the PPAC B signal divided by a factor $10^{4}$. In the figure the shadow of the $25-\mathrm{mm}-$ diameter collimator located behind the PPAC B can be seen. The FWHMs of the ${ }^{15} \mathrm{O}$ beam spot on PPAC B were 7.3 and $10.2 \mathrm{~mm}$ in the horizontal and vertical planes, respectively. The FWHM $1 \mathrm{~mm}$ resolution of the two PPACs employed in the EXOTIC facility, allow us to reconstruct the position of the event on the reaction target with a FWHM $2.3 \mathrm{~mm}$ position resolution.

Figure 36 shows the TOF between the two PPACs, PPAC A and PPAC B, of a ${ }^{8} \mathrm{~B}$ RIB at $E_{l a b}=35 \mathrm{MeV}$ with a FWHM energy spread of $1 \mathrm{MeV}$ [59]. The FWHM of the TOF peak in the figure is equal to $1.60 \mathrm{~ns}$ and is due to the time resolution of both PPAC A and PPAC B, to the considered RIB energy spread and to the intrinsic resolution of the employed electronics. By taking into account the above contributions, the FWHM time resolution of a PPAC 
was found to be 0.86 ns.

The PPAC is able to sustain high rates up to $\sim 4.5 \times 10^{5} \mathrm{~Hz}$ as was demonstrated with a ${ }^{11} \mathrm{C}$ RIB produced at $E_{l a b}=44 \mathrm{MeV}$ with the EXOTIC facility, though with a lower efficiency (by $\sim 7 \%$ ) at this rate because of the delay lines. As mentioned previously, this efficiency loss can be removed by using a multi-hit TDC.

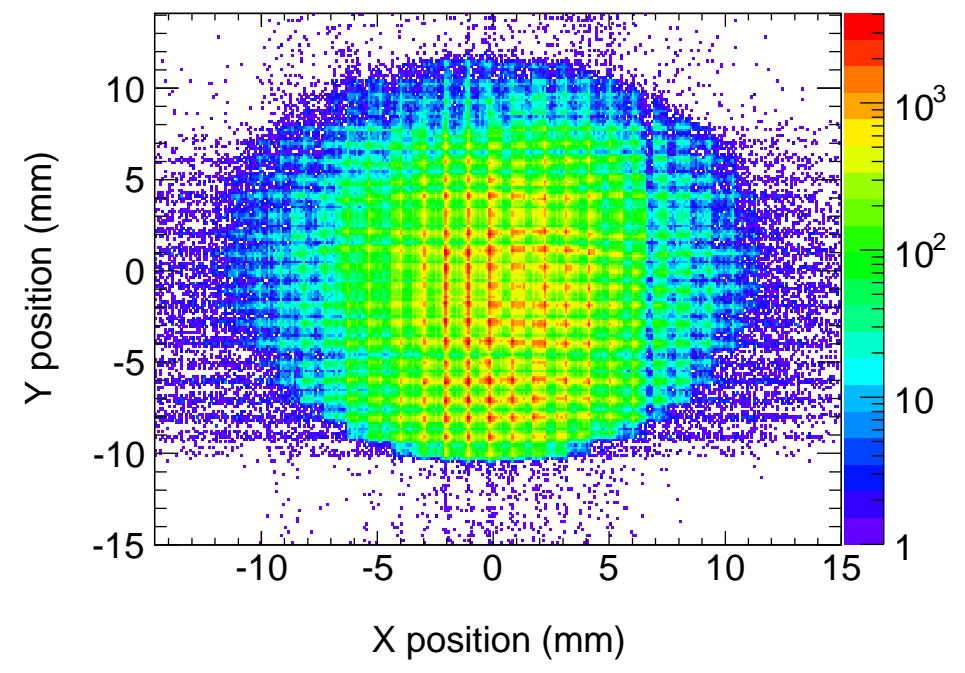

Figure 35. (color online) Profile on PPAC B of a ${ }^{15} \mathrm{O}$ RIB produced with the EXOTIC facility at $E_{l a b}=31 \mathrm{MeV}$.

\subsection{EXPADES}

The performance of the EXPADES telescopes was tested in a true experimental environment at INFN-LNL (Italy). The experimental set-up is sketched in Figure 37. Two 300- $\mu \mathrm{m}$ thick DSSSDs were located in the forward hemisphere. Detector A (B) was placed at a mean polar angle $\theta_{2 L}=49.4^{\circ}\left(\theta_{2 R}=-49.5^{\circ}\right)$ and at a distance of $119.9 \mathrm{~mm}(118.4 \mathrm{~mm})$ from the target. In the backward 


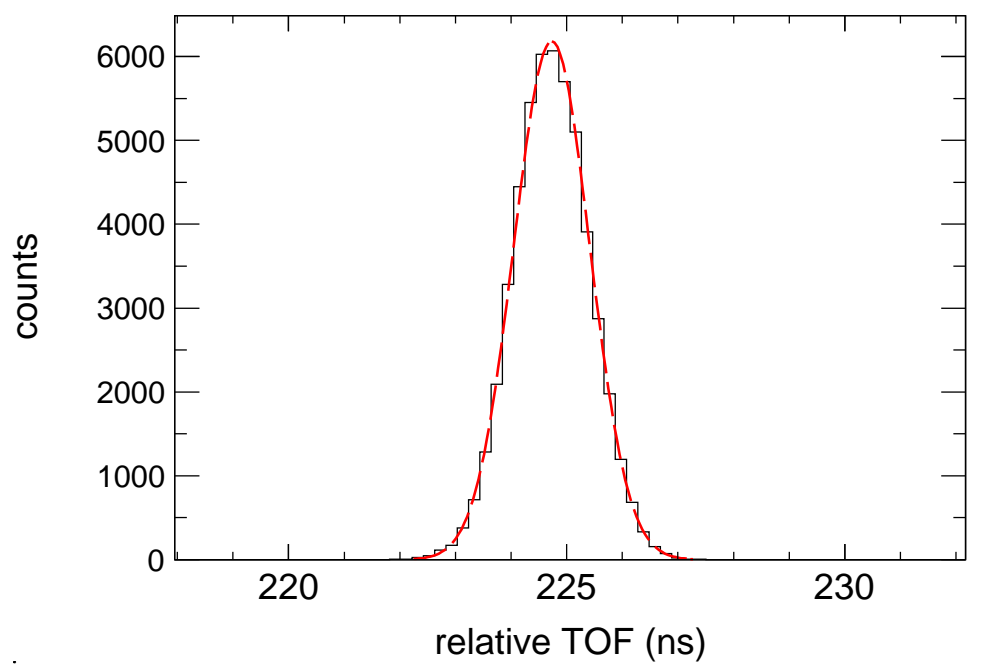

Figure 36. (color online) TOF between PPAC A and PPAC B of a ${ }^{8} \mathrm{~B}$ RIB produced at $E_{l a b}=35 \mathrm{MeV}$ with the EXOTIC facility. The time calibration is not in absolute values. The line represents the Gaussian curve that fits the data. The FWHM of the peak is equal to $1.60 \mathrm{~ns}$ resulting in a PPAC FWHM time resolution of $0.86 \mathrm{~ns}$. For details see text.

hemisphere, a $43 \mu \mathrm{m}$-thick $\Delta E(\mathrm{C})+300 \mu \mathrm{m}$-thick $E_{\text {res }}(\mathrm{D})$ DSSSD telescope was located at a mean polar angle $\theta_{3 R}=-110.8^{\circ}$ and at a distance of $107.3 \mathrm{~mm}$ from the target. A further telescope consisting of an IC followed by a $100 \mu \mathrm{m}-$ thick surface barrier silicon detector (E) was positioned at a mean polar angle $\theta_{3 L}=108.2^{\circ}$ and at $125.3 \mathrm{~mm}$ far from the target. Two additional $100 \mu \mathrm{m}$ thick surface barrier silicon detectors, for beam monitoring and normalization purposes, were positioned at very forward angles: the former $\left(m_{L}\right)$ at $\theta_{1 L}=$ $18.7^{\circ}$ and at $179 \mathrm{~mm}$ from the target, the latter $\left(m_{R}\right)$ at $\theta_{1 R}=-18.7^{\circ}$ and at a distance of $182 \mathrm{~mm}$ from the target. Aluminum disks with a $1 \mathrm{~mm}$-diameter hole in the middle were placed in front of the $m_{L}$ and $m_{R}$ detectors to limit their counting rates. 
Detectors B and C were equipped with the electronics developed for the 40-60 $\mu \mathrm{m}$ DSSSD of EXPADES (16-channel charge-sensitive preamplifiers and the MEGAMP modules) described in Section 3, whereas DSSSDs A and D were connected to the ASIC electronics (VA-TA boards), presented in Section 4. Two ADCs (described in Section 6) were used for the DAQ and all 8 samples per strip were stored to disk. The DAQ dead time was about $25 \%$ and lower than $1 \%$ for a total trigger rate of $1.2 \mathrm{kHz}$ and $700 \mathrm{~Hz}$, respectively. This was due to the presence of a data buffer that should be read more rapidly than is being written. In this case the buffer is never full and the dead time increases linearly with the rate. However, if the buffer is read more slowly than being written, it becomes full resulting in a non-linear increase of the dead time (threshold effect). Better DAQ performance can be achieved by saving to disk a smaller number of samples per strip.

The beam was ${ }^{17} \mathrm{O}$ with an energy varying in the range $40-50 \mathrm{MeV}$ (with a 2.5 MeV step) and with an intensity of about $1 \mathrm{enA}$. The target consisted of a $150 \mu \mathrm{g} / \mathrm{cm}^{2}$-thick ${ }^{58} \mathrm{Ni}$ foil with a $50 \mu \mathrm{g} / \mathrm{cm}^{2}$-thick ${ }^{208} \mathrm{~Pb}$ backing. The thin $\mathrm{Pb}$ layer was added for data normalization purposes. Three collimators with diameter $\phi_{1}=2 \mathrm{~mm}, \phi_{2}=1 \mathrm{~mm}$ and $\phi_{3}=3 \mathrm{~mm}$ were placed $250 \mathrm{~mm}, 30 \mathrm{~mm}$ and $10 \mathrm{~mm}$ upstream the target, respectively, defining a $\phi \sim 1 \mathrm{~mm}$ spot on target. Some runs were performed by using a $200 \mu \mathrm{g} / \mathrm{cm}^{2}$-thick ${ }^{208} \mathrm{~Pb}$ target. In order to show the energy resolution of the DSSSDs for ions with $Z=8$, Figure 38a illustrates the energy spectrum collected by a pixel at $\theta_{2 L}=119.5^{\circ}$ 


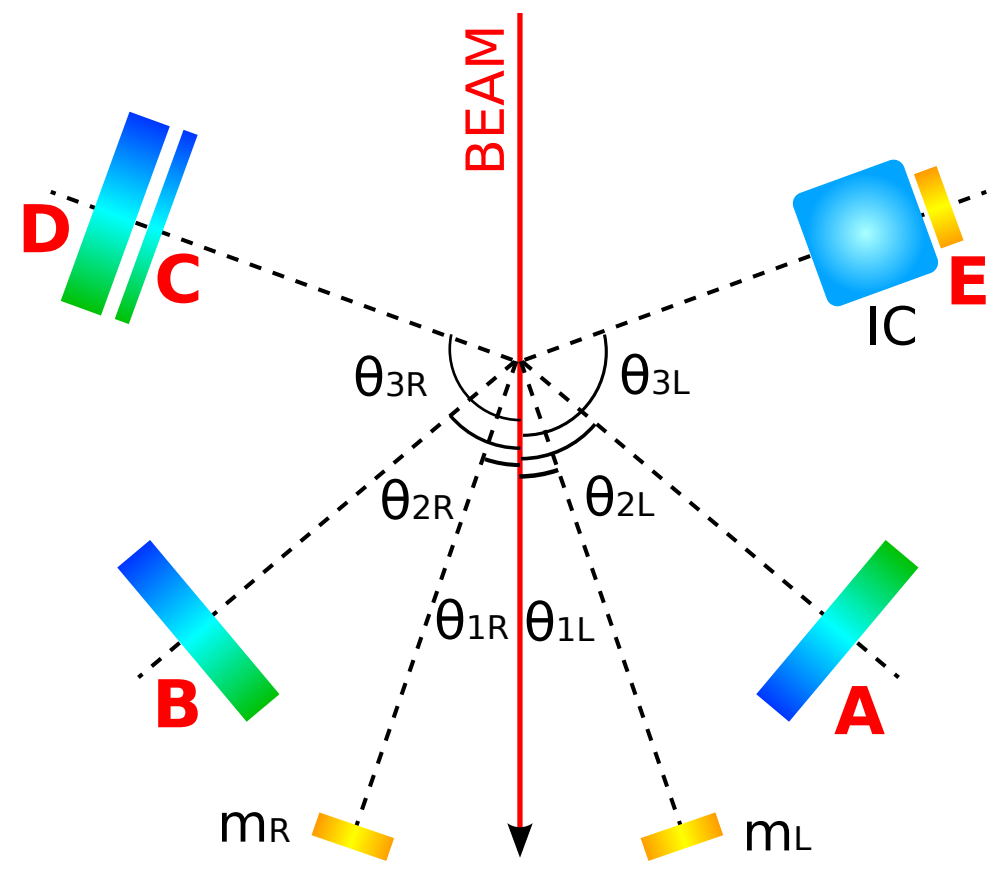

Figure 37. (color online) Schematic view of the experimental set-up used for the in-beam test of the detection array performance. Detectors A, B and D are 300 $\mu$ m-thick DSSSDs; C is a $43 \mu$ m-thick DSSSD; E, $m_{R}$ and $m_{L}$ are $100 \mu$ m-thick surface barrier silicon detectors; IC is an ionization chamber. The displayed polar angles have the following values: $\theta_{1 R}=-18.7^{\circ}, \theta_{1 L}=18.7^{\circ}, \theta_{2 R}=-49.5^{\circ}, \theta_{2 L}=49.4^{\circ}$, $\theta_{3 R}=-110.8^{\circ}, \theta_{3 L}=108.2^{\circ}$. Drawing not in scale.

${ }_{1132}$ of detector $\mathrm{C}$ (43 $\mu$ m-thick, see set up of Figure 37$)$ for the scattering ${ }^{17} \mathrm{O}+$ ${ }_{1133}{ }^{58} \mathrm{Ni},{ }^{208} \mathrm{~Pb}$ at $E_{l a b}=50 \mathrm{MeV}$ while (b) shows the same spectrum for a pixel of ${ }_{1134}$ detector $\mathrm{A}(300 \mu \mathrm{m}$-thick, see set up of Figure 37$)$ at $\theta_{2 L}=49.4^{\circ}$. We examine 1135 the scattering of ${ }^{17} \mathrm{O}$ on the ${ }^{208} \mathrm{~Pb}$ backing because at this incident energy the 1136 scattering is purely Rutherford, the incident energy being below the Coulomb 1137 barrier of the projectile and target nuclei.

${ }_{1138}$ The FWHM overall energy resolution of the A detector (see Figure 38b that 
- the measured electronic noise $\delta E_{\text {exp,noise }}=177 \mathrm{keV}$;

- the statistical fluctuation of the number of created charge carriers in the silicon detector $\delta E_{\text {stat }}=10 \mathrm{keV}$, calculated according to Equation (3) with $F=0.11$ and $w=3.6 \mathrm{eV}$;

- the ion energy broadening $\delta E_{k i n}=69 \mathrm{keV}$ due to the fact that the reaction can take place at any point in the target and

- the energy straggling in the target and the detector dead layer $\delta E_{s t r}=158$ $\mathrm{keV}$ (calculated according to Equation (4)).

By summing the above contributions in quadrature we obtain the FWHM expected overall resolution for the elastic scattering of ${ }^{17} \mathrm{O}$ ions $\delta E_{t o t, t h}=247$ $\mathrm{keV}$, to be compared with the experimental value $\delta E_{\text {exp }}=352 \mathrm{keV}$. By subtracting the electronic noise, the ion energy broadening and the straggling contribution from the experimental resolution $\delta E_{\exp }$ we obtain the detector FWHM intrinsic resolution for ${ }^{17} \mathrm{O}$ ions, $\delta E_{\text {intr }}=251 \mathrm{keV}$ resulting in $0.55 \%$ at $E_{17}=45.352 \mathrm{MeV}$. The FWHM energy resolution that we obtain for the chain (300 $\mu$ m-thick DSSSD+ electronics) is $307 \mathrm{keV}(0.68 \%)$. 
$1168 \mathrm{keV}$;

The FWHM overall energy resolution of the C detector (see Figure 38a that displays the energy spectrum collected by a pixel) was found to be $\delta E_{\text {exp }}=$ $493 \mathrm{keV}$ resulting in $R_{e x p}=1.37 \%$ at $E_{17}=35.915 \mathrm{MeV}$ (the energy loss in the target and the detector dead layer, was calculated with LISE). This value was obtained with $0.5 \mu$ s shaping time. As previously, the following contributions (FWHM) are expected to the overall resolution:

- the measured electronic noise $\delta E_{\text {exp }, \text { noise }}=57 \mathrm{keV}(0.5 \mu$ s shaping time);

- the statistical fluctuation of the number of created charge carriers $\delta E_{\text {stat }}=9$

- the ion energy broadening $\delta E_{k i n}=294 \mathrm{keV}$ due to the fact that the reaction can take place at any point in the target and

- the energy straggling in the target and the detector dead layer $\delta E_{s t r}=201$ $\mathrm{keV}$ (calculated according to Equation (4)).

By summing the above contributions in quadrature we obtain the FWHM expected overall resolution for the elastic scattering of the ${ }^{17} \mathrm{O}$ ions $\delta E_{t o t, t h}=$ $361 \mathrm{keV}$, to be compared with the experimental value $\delta E_{\text {exp }}=493 \mathrm{keV}$. By subtracting the electronic noise, the ion energy broadening and the straggling contribution from the experimental overall energy resolution, $\delta E_{\text {exp }}$, we obtain the detector FWHM intrinsic resolution for ${ }^{17} \mathrm{O}$ ions, $\delta E_{\text {intr }}=336 \mathrm{keV}(0.94 \%$ at $\left.E_{17}=35.915 \mathrm{MeV}\right)$. The FWHM energy resolution that we derive for the chain (43 $\mu$ m-thick DSSSD+electronics) is $341 \mathrm{keV}(0.95 \%)$. 
By comparing the FWHM intrinsic resolution of detectors A (251 keV) and C (336 keV) with that obtained in [60] (see Fig.1) with surface barrier silicon detectors for ${ }^{16} \mathrm{O}$ ions of comparable energy, namely $130 \mathrm{keV}$ after subtraction of the electronic noise, we deduce that some additional contributions to the obtained energy resolution exist in our case, such as, for instance, target nonuniformity as also discussed in [61]. In this paper the authors investigated the degradation of the beam energy distribution due to non-uniformities in the target thickness in fusion excitation function measurements employing the stacked target technique. The above hypothesis is also supported by the observation that the difference between the two intrinsic resolution values is more relevant for detector $\mathrm{C}$ (placed at a backward polar angle), where ions enter the detector after crossing more material layers, than for detector A. A 15-20\% non-uniformity of the $\mathrm{Ni}$ and $\mathrm{Pb}$ targets (upper limit), would provide an additional contribution, which would significantly decrease the difference between the intrinsic resolution measured in our work and that obtained in [60] for $Z=8$ ions. Another possible explanation of the above resolution difference is an underestimation of the straggling term. Nevertheless, we remark that the achieved energy resolution for ${ }^{17} \mathrm{O}$ ions for both detectors $\mathrm{A}$ and $\mathrm{C}$ (detector+electronics) is within the desired value $(\sim 400 \mathrm{keV})$ needed for the separation of the projectile elastic and inelastic scattering processes in direct kinematics (this resolution is requested for the ${ }^{17} \mathrm{~F}$ projectile impinging on a ${ }^{58} \mathrm{Ni}$ or ${ }^{208} \mathrm{~Pb}$ target). However, we remind that in experiments with in-flight RIBs, the overall experimental energy resolution is mainly limited by the RIB 


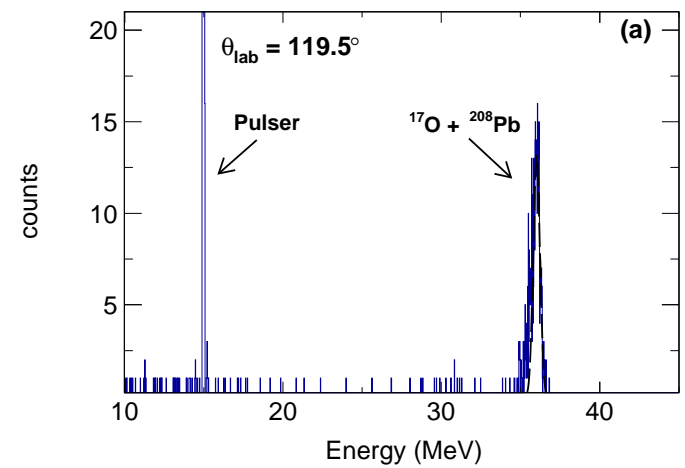
RIB.

energy spread and by the energy loss and straggling into the target, whose thickness is often a compromise between the collection of a suitable counting statistics and an acceptable kinematic broadening and energy spread of the

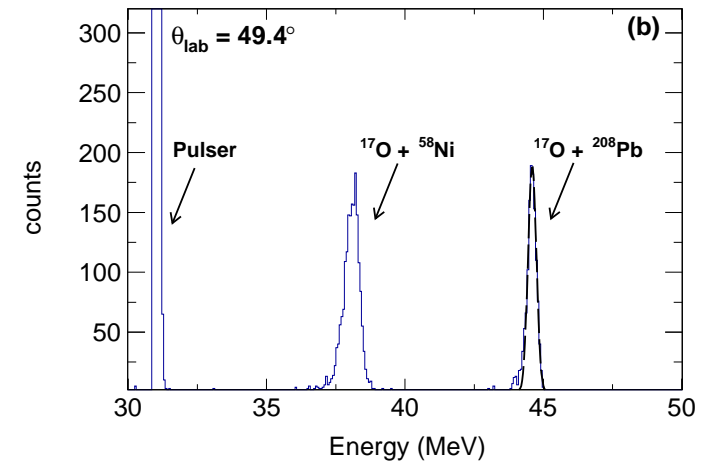

Figure 38. (color online) (a) Energy spectrum collected by a pixel of detector C (43 $\mu \mathrm{m}$-thick, see set up of Figure 37) for the scattering ${ }^{17} \mathrm{O}+{ }^{58} \mathrm{Ni},{ }^{208} \mathrm{~Pb}$ at $E_{l a b}=50$ $\mathrm{MeV}$. The detector was equipped with the electronics developed for the $40 / 60 \mu \mathrm{m}$ DSSSD of EXPADES (16-channel charge-sensitive preamplifiers and the MEGAMP modules described in Section 3); (b) As in (a) for a pixel of detector A (300 $\mu$ m-thick, see set up of Figure 37) that was connected to the ASIC electronics (VA-TA boards, presented in Section 4). In (a) the peak corresponding to the ${ }^{17} \mathrm{O}$ scattering from the ${ }^{58} \mathrm{Ni}$ target should be positioned very close to the pulser peak (as seen from the strip data), however the statistics of the pixel was too low and the peak too broad, due to kinematic effects, to be visible in the figure.

\subsubsection{Particle identification}

An example of the detection array capability in the identification of the detected particles is given in this section. 
1211 The left-hand side of Figure 39 shows a $\Delta E-E_{\text {res }}$ correlation plot obtained 1212 by using an IC as $\Delta E$ stage and the silicon detector $\mathrm{E}$ as $E_{\text {res }}$ layer (see ${ }_{1213}$ experimental set-up of Figure 37) for the ${ }^{17} \mathrm{O}+{ }^{208} \mathrm{~Pb}\left(200 \mu \mathrm{g} / \mathrm{cm}^{2}\right.$-thick 1214 target) reaction at $E_{l a b}=87 \mathrm{MeV}$. The E detector was placed at $211 \mathrm{~mm}$ from 1215 the target, defining a $\pm 3.3^{\circ}$ opening angle for the trajectories of the ions 1216 registered in coincidence with the IC. The IC was operated with $\mathrm{CF}_{4}$ gas at ${ }_{1217}$ a pressure of 60 mbar. The intense peak due to ${ }^{17} \mathrm{O}$ elastically scattered ions 1218 is clearly visible in the figure. The nitrogen $(Z=7)$ and carbon $(Z=6)$ lines 1219 from stripping reaction mechanisms are also easily distinguishable. Finally, in 1220 the bottom of the plot one can observe the helium region, dominated by $\alpha$ 1221 particles emitted after a fusion reaction.

1222 On the right-hand side of Figure 39 we display a zoom of the $\Delta E-E_{\text {res }}$ ${ }_{1223}$ correlation plot obtained by using an IC as $\Delta E$ stage and a strip of a 300 ${ }_{1224} \mu \mathrm{m}$-thick DSSSD of the EXPADES array as $E_{\text {res }}$ layer for the reaction ${ }^{7} \mathrm{Li}+$ ${ }^{1225}{ }^{12} \mathrm{C}$ at $E_{l a b}=10 \mathrm{MeV}$. In this experiment, the $\mathrm{IC}$ was operated with $\mathrm{C}_{4} \mathrm{H}_{10}$ gas 1226 at 90 mbar pressure. One can appreciate on the figure the good separation of 1227 the hydrogen isotopes produced in the reaction.

${ }_{1228}$ In order to evaluate the achieved IC energy loss resolution for heavy ions, 1229 Figure 40 displays the energy loss spectrum of ${ }^{17} \mathrm{O}$ ions elastically scattered ${ }_{1230}$ from the ${ }^{208} \mathrm{~Pb}$ target (corresponding to the events included in the red contour ${ }_{1231}$ of Figure 39, left-hand side). The ${ }^{17} \mathrm{O}$ ions enter the IC gas with an energy of ${ }_{1232} 68.4 \mathrm{MeV}$ (energy losses in the target and the mylar window were calculated 
with LISE) and they loose $\Delta E=5.865 \mathrm{MeV}$ in the gas, that is about $9 \%$ of

their total energy. A FWHM overall experimental resolution $\delta(\Delta E)_{\text {exp }}=343$

$\mathrm{keV}(5.8 \%)$ was obtained (at $1 \mu$ s shaping time). The following contributions

(FWHM) are expected to the overall resolution:

- electronic noise: The measured noise (at $1 \mu$ s shaping time), obtained with the signal of a pulser, $\delta(\Delta E)_{\text {exp,noise }}=50 \mathrm{keV}$;

- the statistical fluctuation of the number of created charge carriers in the gas $\delta(\Delta E)_{\text {stat }}=19 \mathrm{keV}$;

- variation in $\Delta E$, due to energy straggling in the gas $\delta(\Delta \mathrm{E})_{s t r}=256 \mathrm{keV}$ (calculated according to Equation (4)) and

- $\delta(\Delta E)_{k i n}=82 \mathrm{keV}$, due to the broadening of the ion energy entering the gas: the considered opening angle, the straggling in the mylar window and the fact that the reaction can take place at any point in the target were taken into account.

By adding the above contributions, we obtain the FWHM expected overall resolution $\delta(\Delta E)_{t o t, t h}=274 \mathrm{keV}$, corresponding to $R_{t o t, t h}=4.7 \%$ at $\Delta E=5.865$ $\mathrm{MeV}$. This value is slightly different from the experimental one $\delta(\Delta E)_{\text {exp }}=343$ keV. A 15-20\% non-uniformity of the target would result in a negligible $\Delta \mathrm{E}$ variation, thus, the difference could be attributed to underestimation of the straggling term in the gas or to non-uniformities in charge collection and to some inefficiency of the Frisch grid. For this last effect, we notice that in spite of the Frisch grid and of the guard rings employed to maintain uniform the 

1273 [39]).

1274 1275

IC field, some dependence of the collected height signal on the position of the ionizing event with respect to the anode remains (this effect was met also in $[36 ; 37 ; 38]$ where similar devices were used). Although in this measurement such a contribution cannot be evaluated since the silicon detector is not segmented, in the experiments performed with the EXPADES array, the DSSSDs behind the IC allow us to correct for any remnant contribution of this kind because of the requested coincidence of the IC ionizing event with the DSSSD pixels (each pixel defines an opening angle of the particle trajectories less than $1^{\circ}$ for $15 \mathrm{~cm}$ distance from the target) improving, thus, the IC resolution.

By subtracting (in quadrature) from the overall experimental value $\delta(\Delta E)_{\text {exp }}=343$ keV the $\delta(\Delta E)_{\text {kin }}$ term, we obtain the FWHM resolution of the chain (IC+electronics) that is $333 \mathrm{keV}(5.7 \%)$, while by subtracting further the term $\delta(\Delta E)_{\text {exp }, \text { noise }}$, the IC FWHM intrinsic resolution, $\delta(\Delta E)_{\text {intr }}=329 \mathrm{keV}(5.6 \%)$, is deduced. The charge resolving power of the IC for $Z=8$ ions was found to be $Z / \Delta Z=18.5$, adequate for our purposes.

We remark here that the achieved IC energy loss resolution, $R_{\exp }=5.8 \%$, is compatible with the $\sim 7 \%$ value (FWHM) of an axial-field device for $Z=8$ ions at $\Delta E \sim 8.5 \mathrm{MeV}$ in $\mathrm{P} 10\left(90 \% \mathrm{Ar}+10 \% \mathrm{CH}_{4}\right)$ gas (taken from Fig. 10 of

The IC operating pressure can be chosen in each measurement according to the specific experimental needs. 

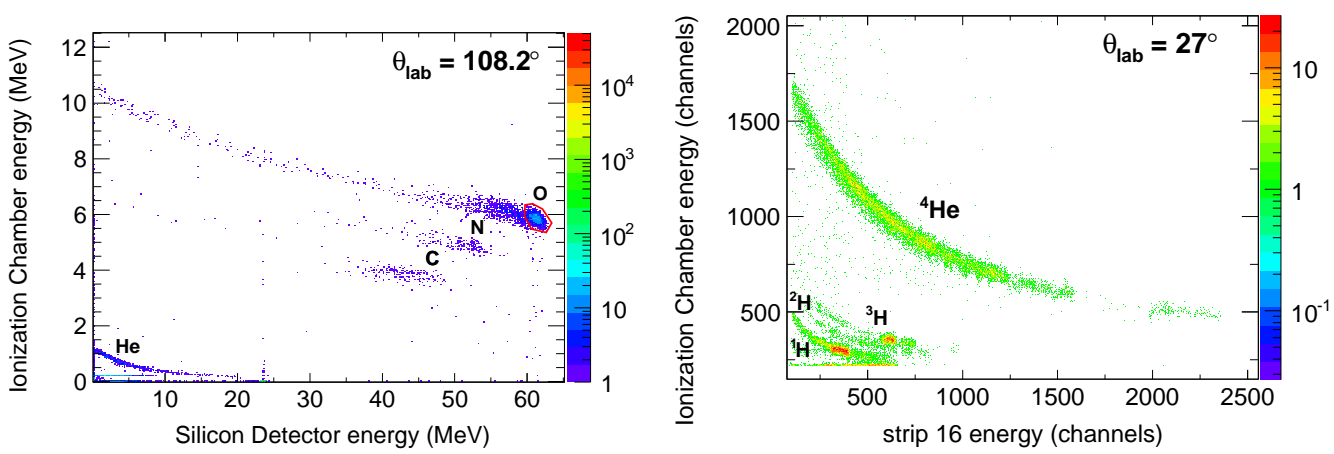

Figure 39. (color online) $\Delta E-E_{\text {res }}$ correlation plot. Left-hand side: ${ }^{17} \mathrm{O}+{ }^{208} \mathrm{~Pb}$ reaction at $E_{l a b}=87 \mathrm{MeV}$. The $\Delta E$ signal was collected from an IC, while the $E_{\text {res }}$ signal was obtained from the detector E (see set-up of Figure 37). The IC was operated with $\mathrm{CF}_{4}$ gas at 60 mbar pressure. Right-hand side: zoom on light ions detected in the reaction ${ }^{7} \mathrm{Li}+{ }^{12} \mathrm{C}$ at $E_{l a b}=10 \mathrm{MeV}$. The $\Delta E$ signal was collected from an IC, while the $E_{\text {res }}$ signal was obtained from a vertical strip of a $300 \mu$ m-thick DSSSD of the EXPADES array. The IC was operated with $\mathrm{C}_{4} \mathrm{H}_{10}$ gas at 90 mbar pressure.

Figure 41 shows a $\Delta E-E_{\text {res }}$ plot originated by the interaction of a ${ }^{3} \mathrm{He}-$ 


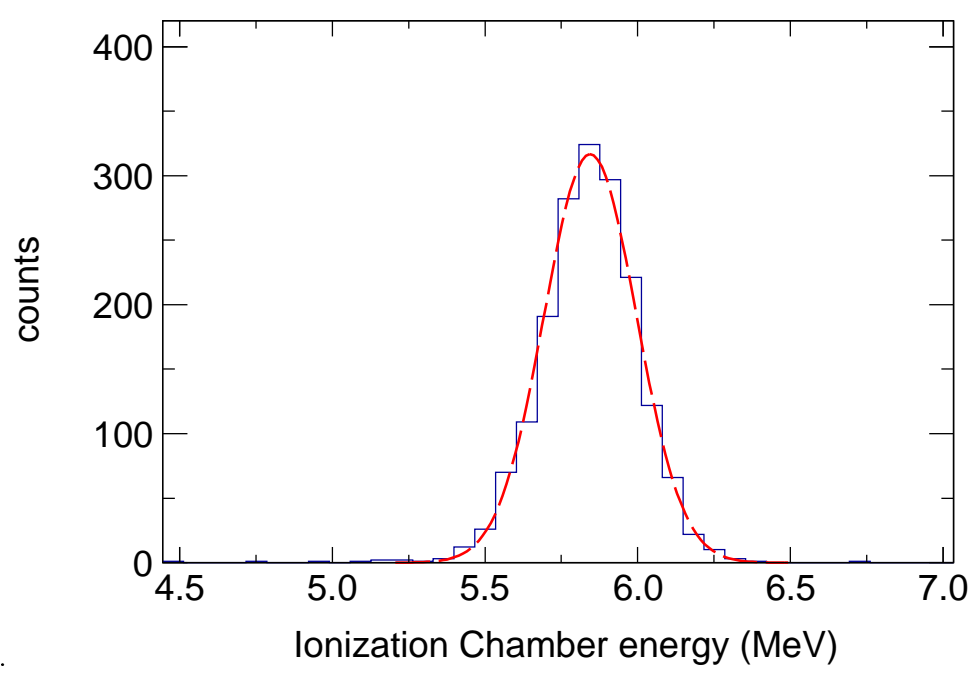

Figure 40. (color online) Energy loss spectrum of ${ }^{17} \mathrm{O}$ ions elastically scattered from a ${ }^{208} \mathrm{~Pb}$ target (events included in the red contour of Figure 39-left). The line is a fit of the data with a Gaussian curve. At $\Delta E=5.865 \mathrm{MeV}$ a FWHM overall experimental resolution $\delta(\Delta E)_{\text {exp }}=343 \mathrm{keV}(5.8 \%)$ was obtained and a FWHM intrinsic resolution $\delta(\Delta E)_{\text {intr }}=329 \mathrm{keV}(5.6 \%)$.

During the experiment, the electronic thresholds of the detectors are set just above the noise that is kept as low as possible. This is achieved thanks to 


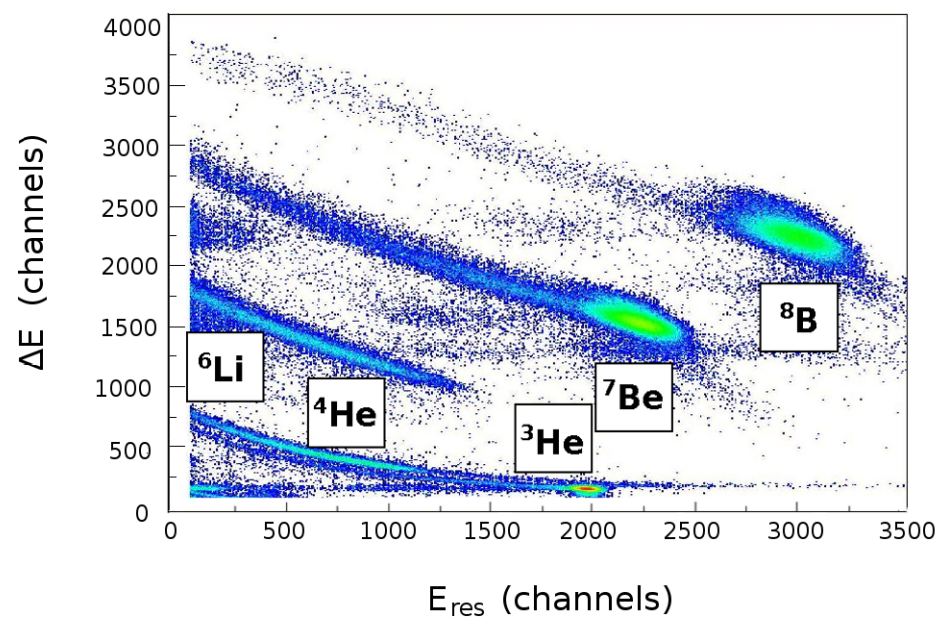

Figure 41. (color online) $\Delta E-E_{\text {res }}$ correlation plot with a two-stage DSSSD telescope for the reaction between the cocktail beam ${ }^{3} \mathrm{He}^{-}{ }^{7} \mathrm{Be}-{ }^{8} \mathrm{~B}$ and a ${ }^{208} \mathrm{~Pb}$ target. Beam energies were $\sim 50, \sim 37$ and $\sim 22 \mathrm{MeV}$ for ${ }^{8} \mathrm{~B},{ }^{7} \mathrm{Be}$ and ${ }^{3} \mathrm{He}$, respectively.

\section{Summary}

We presented in this work the experimental set-up of the RIB in-flight facility EXOTIC: a) two PPACs employed for the event-by-event tracking of the produced RIB and for TOF measurements and b) EXPADES, a new compact high-granularity telescope array, especially tailored for experiments involving light RIBs. Besides compactness, additional advantages of the array are flexibility and portability.

EXPADES consists of eight $40 / 60 \mu \mathrm{m}(\Delta E)-300 \mu \mathrm{m}\left(E_{\text {res }}\right)$ DSSSD telescopes. Eight additional ICs can be used as an alternative $\Delta E$ stage or to build up more complex triple telescopes. In the specific case of experiments where detection of more energetic particles is needed, $1 \mathrm{~mm}$-thick DSSSDs for 
the $E_{\text {res }}$ stage were recently purchased to substitute the $300 \mu \mathrm{m}$-thick detectors or as an additional layer. With the combined use of both DSSSDs and ICs, the detection array allows particle identification in the whole energy range of interest for nuclear reactions induced by light RIBs. The DSSSDs have an area of $64 \times 64 \mathrm{~mm}^{2}$ and each side is segmented into 32 strips, defining a $\sim 2 \times 2$ $\mathrm{mm}^{2}$ pixel structure and an angular resolution ranging from $\Delta \theta=0.5^{\circ}$ to $1^{\circ}$, depending on the distance from the reaction target. This allows measurements of coincident particles emitted with a small relative angle and fits well with the experimental request of various measurements for high granularity.

Very innovative readout electronics was designed for both DSSSD stages. 16channel low-noise charge-sensitive preamplifiers and spectroscopy amplifiers, associated with CFDs, peak-and-hold and TAC circuits were developed for the electronic readout of the $\Delta E$ stage, while the use of ASIC-based electronics was undertaken for the $E_{\text {res }}$ layer. Moreover, the system was equipped with completely new ADCs, TSB for handling the proposed trigger signals of the whole experimental set-up and DAQ.

The performance of the PPACs and of the EXPADES array was initially tested offline with standard $\alpha$ sources. A quite remarkable FWHM energy resolution of $0.65 \%$ was obtained at $\mathrm{E}_{\alpha}=5.805 \mathrm{MeV}$ for the chain ( $43 \mu \mathrm{m}$-thick DSSSD $\Delta E$ stage+electronics), dominated by the DSSSD intrinsic resolution (0.59\%). A poorer energy resolution of $1.14 \%$ (at $\mathrm{E}_{\alpha}=5.805 \mathrm{MeV}$ ) was achieved for the chain (300 $\mu \mathrm{m}$-thick DSSSD $E_{\text {res }}$ stage+electronics) due to the electronic noise 


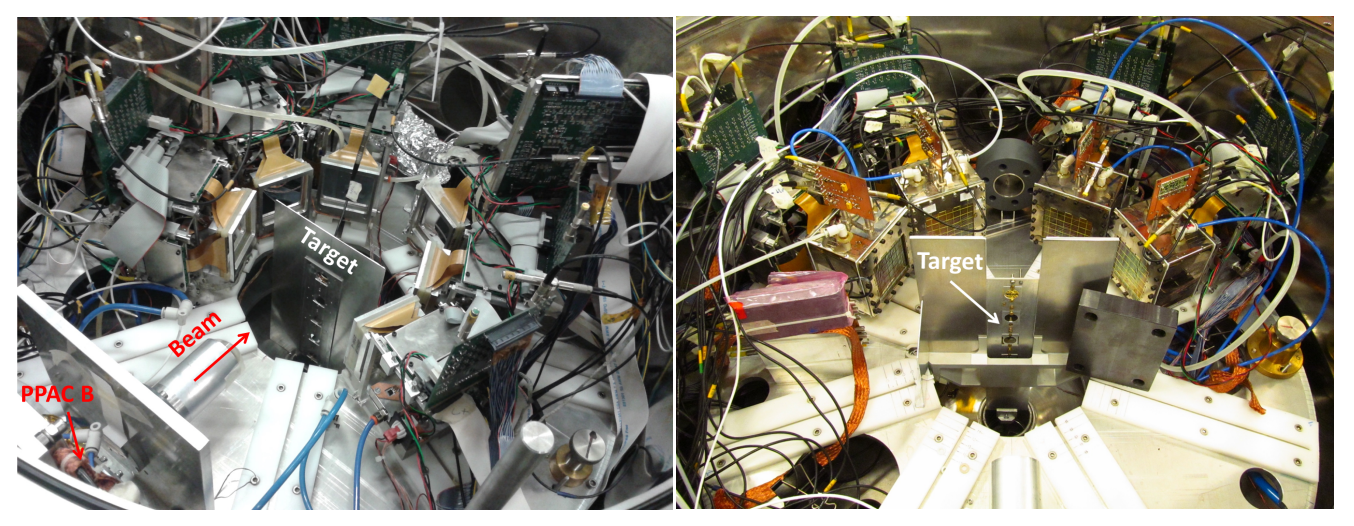

Figure 42. (color online) EXPADES mounted in the reaction chamber of the EXOTIC facility in two configurations. Left: Six two-stage DSSSD telescopes. Right: Four three-stage IC-DSSSD telescopes.

of the ASIC chip (0.57\% DSSSD intrinsic resolution). The FWHM energy loss resolution of the chain (IC+electronics) for an $\alpha$ particle energy loss of $0.996 \mathrm{MeV}$ was found to be $7.3 \%$ (6.5\% FWHM IC intrinsic resolution), close to that of an axial-field device at similar experimental conditions. Moreover, a FWHM time resolution of about $1 \mathrm{~ns}$ for the whole chain $(43 \mu \mathrm{m}$-thick DSSSD+preamplifier+MEGAMP CFD) was obtained.

The PPAC capabilities with light (up to $Z=8$ ) ions were investigated in the first runs employing the EXOTIC RIBs: 0.86 ns FWHM time (intrinsic) resolution and a $1 \mathrm{~mm}$ FWHM position resolution were observed. The above values allow TOF measurements between PPAC and the $\Delta E$ DSSSD of the EXPADES array with a FWHM overall time resolution of about 1.5 ns and reconstruction of the event position on the reaction target with $2.3 \mathrm{~mm}$ FWHM resolution. The PPACs were found to be able to sustain high counting rates up to $\sim 4.5 \times 10^{5} \mathrm{~Hz}$, though with a $\sim 7 \%$ efficiency loss at this high rate due 
to the dead time introduced by the delay lines readout (used for the position determination). This efficiency loss can be removed with the use of a multi-hit TDC.

EXPADES was tested in an in-beam experimental environment by measuring the scattering process for the system ${ }^{17} \mathrm{O}+{ }^{58} \mathrm{Ni},{ }^{208} \mathrm{~Pb}$ at several energies around the Coulomb barrier. The energy resolution (FWHM) of the different detectors for ${ }^{17} \mathrm{O}$ ions was found to be:

- $341 \mathrm{keV}$ for the chain (43 $\mu$ m-thick DSSSD+electronics) that corresponds to $0.95 \%$ resolution at $E=35.915 \mathrm{MeV}$ (detector intrinsic resolution $0.94 \%$ );

- $307 \mathrm{keV}$ for the chain (300 $\mu \mathrm{m}$-thick DSSSD+electronics) corresponding to $0.68 \%$ resolution at $E=45.352 \mathrm{MeV}$ (detector intrinsic resolution $0.55 \%$ );

- $333 \mathrm{keV}$ for the chain (IC+electronics) corresponding to $5.7 \%$ resolution at energy loss $\Delta E=5.865 \mathrm{MeV}$ (detector intrinsic resolution $5.6 \%$ ).

Although the above intrinsic resolution values for $Z=8$ ions include some additional contribution not taken into account in the present work (see discussion in Sections 10.2 and 10.2.1), they are sufficient for the purposes of the envisioned measurements. The achieved telescope resolving power allows us to clearly identify the ions of interest, by considering the combined use of the IC and the $43 \mu \mathrm{m}$-thick DSSSD as $\Delta E$ layer.

Summarizing, both the PPAC performance and the capabilities of the array for particle identification and TOF measurements were found to be adequate for 


\section{References}

[1] T. Kubo et al., Nucl. Instr. and Meth. B 70 (1992) 309; T. Suda J. Phys.: Conf. Ser. 267 (2011) 012008 
[2] The K500-K1200, A Coupled Cyclotron Facility at the NSCL, MSU, MSUCL-939, July 1994

[3] W. Mittig, J. Phys. G: Nucl. Part. Phys. 24 (1998) 1331

[4] H. Geissel et al., Nucl. Instr. and Meth. B 70 (1992) 286

[5] A. Herlert, Nucl. Phys. News 20 (2010) 5

[6] G. C. Ball et al., J. Phys. G: Nucl. Part. Phys. 38 (2011) 024003

[7] A. Aprahamian and M. Wiescher, Nucl. Phys. News 12 (2002) 5

[8] R. Lichtenthäler et al., Nucl. Phys. News 15 (2005) 25

[9] J. Äystö et al., Eur. Phys. J. A 48 (2012) 42

[10] S. Kubono et al., Eur. Phys. J. A 13 (2002) 217

[11] Y. Yanagisawa et al., Nucl. Instr. and Meth. A 539 (2005) 74

[12] H. Yamaguchi et al., Nucl. Instr. and Meth. 589 (2008) 150

[13] V.Z. Maidikov et al., Nucl. Phys. A 746 (2004) 389c

[14] D. Pierroutsakou et al., Eur. Phys. J. Special Topics 150 (2007) 47

[15] F. Farinon et al., Nucl. Instr. and Meth. B 266 (2008) 4097

[16] M. Mazzocco et al., Nucl. Instr. and Meth. B 266 (2008) 4665

[17] M. Mazzocco et al., Nucl. Instr. and Meth. B 317 (2013) 223

[18] Y. Blumenfeld et al., Nucl. Instr. and Meth. A 421 (1999) 471

[19] E. Pollacco et al., Eur. Phys. J. A 25 (s01) (2005) 287

[20] M. Labiche et al., Nucl. Instr. and Meth. A 614 (2010) 439

[21] B. Davin et al., Nucl. Instr. and Meth. A 473 (2001) 302

[22] M.S. Wallace et al., Nucl. Instr. and Meth. A 583 (2007) 302

[23] T. Davinson et al., Nucl. Instr. and Meth. A 454 (2000) 350 
[24] G. Marquínez-Durán et al., Nucl. Instr. and Meth. A 755 (2014) 69

[25] M. Romoli et al., Nucl. Instr. and Meth. B 266 (2008) 4637

[26] E. Strano et al., Nucl. Instr. and Meth. B 317 (2013) 657

[27] L.F. Canto et al., Phys. Rep. 424 (2006) 1; Phys. Rep. 596 (2015) 1

[28] M. Freer, Rep. Prog. Phys. 70 (2007) 21492210

[29] K. Artemov et al., Sov. J. Nucl. Phys. 52 (1990) 408-411 ISSN 0038-5506

[30] J. Walshe et al., J. Phys.: Conf. Ser. 569 (2014) 012052

[31] G. Baur, Phys. Lett. B 178 (1986) 135; R. E. Tribble et al., Rep. Prog. Phys. 77 (2014) 106901

[32] S. Cherubini et al., Phys. Rev. C 92 (2015) 015805

[33] A. Breskin, R. Chechik and N. Zwang, Nucl. Instr. Meth. 165 (1979) 125

[34] http://www.bronkhorst.com

[35] http://www.micronsemiconductor.co.uk/pdf/cat.pdf

[36] K. Rudolph et al., Nucl. Instr. and Meth. A 204 (1983) 407

[37] K. Kusterer et al., Nucl. Instr. and Meth. A 177 (1980) 485

[38] K. Sistemick et al., Nucl. Instr. and Meth. 133 (1976) 163

[39] R.W. Zurmühle and L. Cshihas, Nucl. Instr. and Meth. 203 (1982) 261

[40] S.K. Bandyopadhyaya et al., Nucl. Instr. and Meth. A 278 (1989) 467

[41] K.Y. Chae et al., Nucl. Instr. and Meth. A 751 (2014) 6

[42] L.G. Christophorou et al., Nucl. Instr. and Meth. 163 (1979) 141

[43] A. Pullia et al., IEEE Trans. Nucl. Sci. 48 (2001) 530

[44] C. Boiano et al., 2012 IEEE Nuclear Science Symposium and Medical 
Imaging Conference Record N14-34 (2012) 865

[45] R. Bassini, C. Boiano et al., 2006 IEEE Nuclear Science Symposium Conference Record N14-173 (2006) 507

[46] M. Alderighi et al., IEEE Trans. Nucl. Sci. 52 (2005) 1624 and references therein

[47] G.L. Engel et al., Nucl. Instr. and Meth. A 573 (2007) 418

[48] H. Kumagai et al., Nucl. Instr. and Meth. A 470 (2001) 562

[49] C. Boiano et al., 2007 IEEE Nuclear Science Symposium Conference Record N15-37 (2007) 340

[50] The XDAQ framework, http://www.cern.ch/xdaq

[51] Run Control and Monitoring System, http://cmsdoc.cern.ch/cms/TRIDAS/RCMS/

[52] J. Grebosz, Comp. Phys. Communications 176 (2007) 251

[53] D. Bazin et al., Nucl. Instr. and Meth. A 482 (2002) 307; O.B. Tarasov, D. Bazin, Nucl. Instr. and Meth. B 266 (2008) 4657

[54] F. Hubert et al., Atom. Data Nucl. Data Tables 46 (1990) 1

[55] M. Takahashi et al., Nucl. Instr. and Meth. A 628 (2011) 150

[56] D. Guillemaud-Mueller et al., IEEE Trans. Nucl. Sci. 33 (1) (1986) 343

[57] D. Torresi et al., Nucl. Instr. and Meth. A 713 (2013) 11

[58] L. Grassi et al., Nucl. Instr. and Meth. A 767 (2014) 99

[59] A. Pakou et al., Phys. Rev. C 87 (2013) 014619

[60] C. Lee et al., Nucl. Instr. and Meth. A 432 (1999) 313

[61] M. Fisichella et al., Phys. Rev. C 92 (2015) 064611 
[62] A. Pakou et al., Eur. Phys. J. A (2015) 51: 55

[63] A. Pakou et al., Eur. Phys. J. A (2015) 51: 90

[64] M. Mazzocco et al., NN2015, EPJ Web of Conferences 117, 06006 (2016)

[65] D. Torresi et al., NN2015, EPJ Web of Conferences 117, 08027 (2016)

[66] E. Strano et al., submitted to Phys. Rev. C. 